Universidade de Brasília

Instituto de Ciências Exatas

Departamento de Matemática
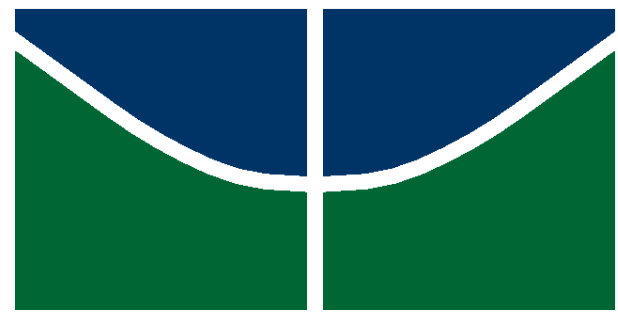

Geometria de Laguerre e representação para superfícies mínimas generalizadas de Laguerre

por

Ricardo Edmundo Zamora Vargas 
Universidade de Brasília

Instituto de Ciências Exatas

Departamento de Matemática

\title{
Geometria de Laguerre e representação para superfícies mínimas generalizadas de Laguerre
}

por

\author{
Ricardo Edmundo Zamora Vargas *
}

Dissertação apresentada ao Departamento de Matemática da Universidade de Brasília, como parte dos requisitos para obtenção do grau de

\section{MESTRE EM MATEMÁTICA}

Brasília, 2015

Comissão Examinadora:

Dr. João Paulo dos Santos - UnB - Orientador

Dr. Carlos Carrión Riveros - UnB - Examinador

Dr. Armando Vasquez Corro - UFG - Examinador

*O autor foi bolsista do CNPq durante a elaboração deste trabalho. 
Aos meus pais, Rosa e Alejandro. 


\section{Agradecimentos}

À minha família, em especial aos meus pais, Rosa Angelina Vargas Solís e Alejandro Edmundo Zamora Tejada, pela educação, incentivo, amor e dedicação. Aos meus irmãos, Rosa e Victor, por trazer alegria à nossa família com meus pequenos sobrinhos, Diego, Kris e Alejandra.

A minha namorada Katherine, pelo amor e apoio em cada passo que juntos temos dado nos últimos quatro anos, animando-me e demonstrando-me seu carinho.

Ao meu orientador, o Professor João Paulo dos Santos, pela amizade, a orientação, e a confiança depositada em mim.

Ao meu professor Ricardo Ruviaro, que no decorrer dos dois anos do mestrado tornou-se um amigo, sempre mostrando seu apoio e sempre dando umas palavras de alento.

Aos meus amigos e colegas, que durante o curso de mestrado fizeram parte de minha vida e que me possibilitam continuar percebendo que a vida não é só trabalhar.

Finalmente, agradeço ao CNPq pelo apoio financeiro concedido durante a elaboração deste trabalho. 
"A medicina cria pessoas doentes, a matemática, pessoas tristes, e a teologia, pecadores".

Martinho Lutero. 


\section{Resumo}

Neste trabalho, apresentamos um estudo da geometria de Laguerre no espaço Euclidiano, apresentando a geometria de esferas e planos orientados, bem como das transformações de Laguerre. Através deste estudo, apresentamos as superfícies e a métrica de Laguerre, cujo elemento de volume é conhecido como funcional de Laguerre. Em seguida, estudamos superfícies mínimas generalizadas de Laguerre, isto é, superfícies que são pontos críticos deste funcional e que admitem pontos isolados com curvatura zero. Analogamente à representação de Weierstrass para superfícies mínimas apresentamos uma representação do tipo Weierstrass que permite descrever globalmente as superfícies mínimas de Laguerre generalizadas usando três dados: uma função meromorfa, uma forma holomorfa e uma função real harmônica. Tal representação é chamada de representação conforme e coincide com a representação de Weierstrass quando a função real harmônica é nula.

Palavras-chave: Geometria de Laguerre, esferas orientadas, superfícies mínimas de Laguerre, superfícies mínimas generalizadas de Laguerre, representação de Weierstrass, representação conforme, métrica de Laguerre completa. 


\section{Abstract}

In this work, we present a study of Laguerre geometry in Euclidean space, presenting the geometry of oriented spheres and planes, as well as the Laguerre transformations. Through this study, we present the Laguerre surfaces and the Laguerre metric, whose volume element is known as the Laguerre functional. Then, study generalized Laguerre minimal surfaces, i.e. surfaces which are critical points of this functional and which allow isolated points with curvature zero. Similarly to the Weierstrass representation for minimal surfaces, we present a kind of Weierstrass representation that allows describe globally generalized Laguerre minimal surfaces using three data: a meromorphic function, a holomorphic form and a real harmonic function. This representation is called conformal representation and coincides with the Weierstrass representation when the harmonic real function is zero.

Key words: Laguerre geometry, oriented spheres, Laguerre minimal surfaces, generalized Laguerre minimal surfaces, Weierstrass representation, conformal representation, complete Laguerre metric. 


\section{Sumário}

Introdução $\quad 2$

1 Preliminares $\quad 4$

1.1 Variedades Riemannianas ... . . . . . . . . . . . . . . . . . 4

1.1.1 Variedades diferenciáveis . . . . . . . . . . . . . . . . . . 4 4

1.1.2 A métrica Riemanniana induzida às subvariedades de $\mathbb{R}^{n} \ldots$. . . . . . . . . . 9

1.1.3 Gradiente, divergência e Laplaciano em subvariedades de $\mathbb{R}^{n}$. . . . . . . . . . . 9

1.2 Superfícies, formas quadráticas e representação de Weierstrass . . . . . . . . . . . . . . . 14

1.2.1 Superfícies regulares . . . . . . . . . . . . . . . . . . . 14

1.2.2 Formas fundamentais . . . . . . . . . . . . . . . . . . . 15

1.2.3 Superfícies de Riemann . . . . . . . . . . . . . . . . . . . 19

1.2.4 Representação de Weierstrass . . . . . . . . . . . . . . . . . . . . 20

2 Geometria de Laguerre $\quad \mathbf{2 5}$

2.1 Geometria das esferas orientadas em $\mathbb{R}^{3} \ldots \ldots \ldots \ldots \ldots \ldots$

2.2 Grupo de transformações de Laguerre sobre $U \mathbb{R}^{3} \ldots \ldots$. . . . . . . . . . . . . . . . 35

2.3 Superfícies de Laguerre em $U \mathbb{R}^{3} \ldots \ldots \ldots \ldots \ldots$. . . . . . . . . . . . . . . . . . . . . 44

3 Teoremas de representação e completude da métrica de Laguerre 5

3.1 Superfícies mínimas generalizadas de Laguerre . . . . . . . . . . . . . . . . . . . 52

3.2 Representação conforme para superfícies-MGL . . . . . . . . . . . . . . . . . . 54

3.3 Completude da métrica de Laguerre . . . . . . . . . . . . . . . . . . . 69

4 Conclusão e futuras linhas de pesquisa $\quad 75$

$\begin{array}{lr}\text { A Superfícies } & \mathbf{8 0}\end{array}$

A.1 Demonstrações de resultados anteriores . . . . . . . . . . . . . . . . . . . 80

A.1.1 Demonstração do Teorema $1.1 \ldots \ldots$. . . . . . . . . . . . . 80

A.1.2 Demonstração do Lema 1.1 . . . . . . . . . . . . . . . . . . . . . . 81

A.1.3 Demonstração do Teorema 1.4 . . . . . . . . . . . . . . . . . . . 82

$\begin{array}{lr}\text { Bibliografia } & 83\end{array}$ 


\section{Introdução}

A geometria de Laguerre constitui uma parte importante na geometria de Lie, nela os teoremas da geometria Euclidiana que dependem somente dos conceitos de esferas e seus contatos tangenciais, tem uma formulação mais natural (ver [8]). Isto é conseguido mediante três etapas. Primeiramente, o espaço Euclidiano é compactificado adicionando um ponto no infinito, de tal forma que os planos podem ser considerados como esferas de raio infinito. Esta extensão é conhecida como geometria de Moebius. Em segundo lugar consideramos os pontos como esferas de raio zero. Finalmente, por razões técnicas, orientam-se esferas e planos em $U \mathbb{R}^{3}$, associando um vetor normal. Estes objetos orientados podem ser considerados como pontos de uma hiperquádrica $\mathrm{Q}^{4}$ no espaço projetivo $\mathbb{R} P^{5}$, que é conhecida como quádrica de Lie.

Um dos mais importantes subgrupos do grupo de transformações da esfera de Lie é o grupo de transformações de Laguerre em $U \mathbb{R}^{3}$, neste subgrupo, estudamos as propriedades invariantes das superfícies. As transformações de Laguerre são as transformações da esfera de Lie que levam planos orientados de $\mathbb{R}^{3} \mathrm{em}$ planos orientados e preservam a distancia tangencial. A geometria das superfícies de Laguerre em $\mathbb{R}^{3}$ foi desenvolvida por Blaschke e sua escola (ver [6]). Nos últimos anos houve um grande interesse no estudo da geometria das superfícies de Laguerre (ver [15], [16]), principalmente desde o ponto de vista local e foi apresentada mais de uma forma de representar as superfícies mínimas de Laguerre em $\mathbb{R}^{3}$ (ver [20], [21]).

Uma das invariantes pelo grupo de transformações de Laguerre é o funcional

$$
L(X)=\int_{S} \frac{H^{2}-K}{K} d S,
$$

onde $X: S \rightarrow \mathbb{R}^{3}$ é uma imersão de uma superfície orientada $S$ com curvatura Gaussiana não nula e curvatura média $H$. Os pontos críticos (superfícies críticas) do funcional $L$ são chamadas superfícies mínimas de Laguerre. O estudo de tais superfícies iniciou com J. Weingarten em 1888 (ver [2]), e posteriormente desenvolvido por Blaschke desde o ano 1924 (ver [6], [3], [4], [5]).

A equação de Euler-Lagrange para superfícies mínimas de Laguerre em (1) foi dada por J. Weingarten como segue

$$
\Delta^{\mathrm{III}}\left(\frac{H}{K}\right)=0
$$


onde $\Delta^{\mathrm{III}}$ é o Laplaciano em relação à terceira forma fundamental III. Claramente, as superfícies mínimas Euclidianas em $\mathbb{R}^{3}$ são superfícies mínimas de Laguerre, no entanto, existem superfícies mínimas de Laguerre em $\mathbb{R}^{3}$ que não são mínimas no sentido Euclidiano. Diversos resultados sobre superfícies mínimas de Laguerre podem-se achar em [6], [15] e [16].

Embora uma superfície mínima de Laguerre seja definida como aquela com curvatura Gaussiana não nula satisfazendo (2), é possível generalizar o conceito de minimalidade para superfícies com curvatura Gaussiana nula em um conjunto isolado de pontos, alguns resultados foram obtidos recentemente por Aledo, Juan A., Gálvez, José e A., Lozano, Victorino (ver [1]) chamadas superfícies mínimas generalizadas de Laguerre, fazendo um estudo das suas propriedades e da sua representação.

O presente trabalho baseia-se principalmente em [13], [21] e [1], os dois últimos apresentam representações para superfícies mínimas e mínimas generalizadas de Laguerre, respectivamente. No primeiro caso em um aspecto local e no segundo em um aspecto global.

Este trabalho está dividido em 4 capítulos e um apêndice, a saber:

\section{- Capítulo 1: Preliminares}

Neste capítulo apresentamos as definições e resultados básicos sobre superfícies e variedades diferenciáveis que usaremos no desenvolvimento do trabalho. Estabelecemos também algumas notações e propriedades a serem generalizadas nos capítulos posteriores. As referencias são [17] e [10].

\section{- Capítulo 2: Geometria de Laguerre}

Neste capítulo apresentamos os conceitos e resultados fundamentais da geometria de Laguerre, fazemos um estudo detalhado da geometria das esferas orientadas em $\mathbb{R}^{3}$ e definimos o grupo de transformações de Laguerre que é um subgrupo importante das transformações da esfera de Lie (ver [8]). Fazemos um estudo das superfícies de Laguerre e apresentamos a métrica e o funcional de Laguerre.

\section{- Capítulo 3: Teoremas de representação e completude da métrica de Laguerre}

Neste capítulo apresentamos uma análise global das superfícies mínimas de Laguerre e estendemos seu estudo para o caso em que a superfície tem curvatura Gaussiana nula em um conjunto de pontos isolados, definindo-as como superfícies mínimas generalizadas de Laguerre. Apresentamos uma representação conforme (global) das superfícies mínimas de Laguerre em termos de uma função meromorfa, uma forma holomorfa e uma função real harmônica e exibimos vários exemplos de como essa representação é aplicada.

\section{- Capítulo 4: Conclusão e futuras linhas de pesquisa}

Neste capítulo são apresentadas as conclusões do trabalho e alguns resultados relacionados que mostram o desenvolvimento da área de pesquisa. Finalizamos com um problema aberto que versa sobre a geração de duas superfícies simétricas que preservam áreas a partir de uma superfície mínima de Laguerre, mostramos alguns gráficos das superfícies simétricas geradas computacionalmente como sendo uma demonstração empírica que posteriormente ajudará a inferir uma possível formalização analítica da demonstração. 


\section{Capítulo 1 \\ Preliminares}

Neste capítulo apresentamos as definições e resultados da geometria Riemanniana a serem utilizados ao longo deste trabalho. Começamos com definições relacionadas às variedades diferenciáveis e em seguida estudamos as superfícies diferenciáveis, em particular, as superfícies Riemannianas. Por serem considerados conhecimentos básicos, alguns resultados estão em forma de afirmação no decorrer do texto e sem demonstrações. Estas afirmações e suas respectivas demonstrações podem ser encontradas na literatura básica da geometria tais como [10] e [24]. Quanto a diferenciabilidade de uma função, adotamos a convenção de ser de classe $C^{\infty}$.

\subsection{Variedades Riemannianas}

\subsubsection{Variedades diferenciáveis}

Definição 1.1. Uma variedade diferenciável de dimensão n é um conjunto $M$ e uma familia de aplicações biunivocas $X_{\alpha}: U_{\alpha} \subset \mathbb{R}^{n} \rightarrow M$ de abertos $U_{\alpha}$ de $\mathbb{R}^{n}$ em $M$ tais que:

1. $\cup_{\alpha} X_{\alpha}\left(U_{\alpha}\right)$.

2. Para todo par $\alpha, \beta$, com $X_{\alpha}\left(U_{\alpha} \cap\left(U_{\beta}\right)=W \neq \emptyset\right.$, os conjuntos $X_{\alpha}^{-1}(W)$ e $X_{\beta}^{-1}(W)$ são abertos em $\mathbb{R}^{n}$ e as aplicações $X_{\beta}^{-1} \circ X_{\alpha}$ são diferenciáveis.

3. A família $\left\{\left(U_{\alpha}, X_{\alpha}\right)\right\}$ é máxima relativamente às condições 1 e 2.

$O \operatorname{par}\left(U_{\alpha}, X_{\alpha}\right)$ (ou a aplicação $X_{\alpha}$ ) com $p \in X_{\alpha}\left(U_{\alpha}\right)$ é chamado uma parametrização (ou sistema de coordenadas) de $M$ em $p ; X_{\alpha}$ é então chamada uma vizinhança coordenada em $p$. Uma família $\left\{\left(U_{\alpha}, X_{\alpha}\right)\right\}$ satisfazendo 1 e 2 é chamada uma estrutura diferenciável em $M$.

De agora em diante, quando indicarmos uma variedade por $M^{m}$, o índice $m$ indicará a dimensão de $M$. Considere em $M$ a topologia induzida pela estrutura diferenciável. No decorrer deste trabalho, as variedades diferenciáveis serão sempre de Hausdorff e com base enumerável. Isto significa que dados dois pontos distintos de $M$ existem vizinhanças destes dois pontos que não se interceptam e que $M$ pode ser coberta por uma quantidade enumerável de vizinhanças coordenadas. 
Exemplo 1.1. Em $M=\mathbb{R}^{n+1} \backslash\{0\}$, definimos a relação de equivalência $\sim$ por:

$$
x \sim y \Leftrightarrow y=t x, \text { para algum } t \neq 0 .
$$

$O$ espaço quociente $\mathbb{R} P^{n}=M / \sim$ chama-se espaço projetivo real. $\mathbb{R} P^{n}$ é uma variedade diferenciável de dimensão n. Geometricamente, cada classe $[L] \in \mathbb{R} P^{n}$ pode ser identificado com a reta em $\mathbb{R}^{n+1} q u e$ passa pela origem cuja direção é dada pelo vetor $x$.

Definição 1.2. Sejam $M_{1}^{n}$ e $M_{2}^{m}$ variedades diferenciáveis. Uma aplicação $\varphi: M_{1} \rightarrow M_{2}$ é diferenciável em $p \in M$ se dada uma parametrização $Y: V \subset \mathbb{R}^{m} \rightarrow M_{2}$ em $\varphi(p)$ existe uma parametrização $X: U \subset \mathbb{R}^{n} \rightarrow M_{1}$ em p tal que $\varphi(X(U)) \subset Y(V)$ e a aplicação

$$
Y^{-1} \circ \varphi \circ X: U \subset \mathbb{R}^{n} \rightarrow \mathbb{R}^{m}
$$

é diferenciável em $X^{-1}(p)$. Dizemos que $\varphi$ é diferenciável em um aberto de $M_{1}$ se é diferenciável em todos os pontos deste aberto.

Decorre da condição 2 da Definição 1.1 que a definição dada é independente da escolha das parametrizações. A aplicação (1.1) é chamada a expressão de $\varphi$ nas parametrizacões $X$ e $Y$.

Definição 1.3. Seja $M$ uma variedade diferenciável. Uma aplicação diferenciável $\alpha:(-\varepsilon, \varepsilon) \rightarrow M$ é chamada uma curva (diferenciável) em $M$. Suponha que $\alpha(0)=p \in M$, e seja $\mathcal{D}(M)$ o conjunto das funções de $M$ diferenciáveis em $p$. O vetor tangente à curva $\alpha$ em $t=0$ é a função $\alpha^{\prime}(0): \mathcal{D}(M) \rightarrow \mathbb{R}$ dada por

$$
\alpha^{\prime}(0) f=\left.\frac{d(f \circ \alpha)}{d t}\right|_{t=0}, \quad f \in \mathcal{D}(M)
$$

Um vetor tangente a $M$ em $p$ é o vetor tangente em $t=0$ de alguma curva $\alpha:(-\varepsilon, \varepsilon) \rightarrow M$ com $\alpha(0)=p$. $O$ conjunto dos vetores tangentes a $M$ em p será indicado por $T_{p} M$.

Se escolhemos uma parametrização $X: U \rightarrow M^{n}$ em $p=X(0)$, podemos exprimir a função $f$ e a curva $\alpha$ nesta parametrização por

$$
f \circ X(q)=f\left(u_{1}, \cdots, u_{n}\right), \quad q=\left(u_{1}, \cdots, u_{n}\right) \in U
$$

$$
X^{-1} \circ \alpha(t)=\left(u_{1}(t), \cdots, u_{n}(t)\right)
$$

respectivamente. Portanto, restringindo $f$ a $\alpha$, obtemos

$$
\begin{aligned}
\alpha^{\prime}(0) f & =\left.\frac{d(f \circ \alpha)}{d t}\right|_{t=0}=\left.\frac{d}{d t} f\left(u_{1}(t), \cdots, u_{n}(t)\right)\right|_{t=0} \\
& =\sum_{i=1}^{n} u_{i}^{\prime}(0)\left(\frac{\partial f)}{\partial u_{i}}\right) \\
& =\left(\sum_{i=1}^{n} u_{i}^{\prime}(0)\left(\frac{\partial}{\partial u_{i}}\right)_{0}\right) f .
\end{aligned}
$$


Assim, o vetor $\alpha^{\prime}(0)$ pode ser expresso na parametrização $X$ por

$$
\alpha^{\prime}(0)=\sum_{i=1}^{n} u_{i}^{\prime}(0)\left(\frac{\partial}{\partial u_{i}}\right)_{0}
$$

Observe que $\left(\frac{\partial}{\partial u_{i}}\right)_{0}$ é o vetor tangente em $p$ à "curva coordenada"

$$
u_{i} \rightarrow X\left(0, \cdots, 0, u_{i}, 0, \cdots, 0\right)
$$

A expressão (1.2) mostra que o vetor tangente a uma curva $\alpha$ em $p$ depende apenas das derivadas de $\alpha$ em um sistema de coordenadas. Decorre também de (1.2) que o conjunto $T_{p} M$, com as operações usuais de funções, forma um espaço vetorial de dimensão $n$, e que a escolha de uma parametrização $X: U \rightarrow M$ determina uma base associada $\left\{\left(\frac{\partial}{\partial u_{i}}\right)_{0}\right\}_{i=1}^{n}$ em $T_{p} M$. É imediato que a estrutura linear em $T_{p} M$ assim definida não depende da parametrização $X$. O espaço vetorial $T_{p} M$ é chamado o espaço tangente de $M$ em $p$.

Proposição 1.1. Sejam $M_{1}^{n}$ e $M_{2}^{m}$ variedades diferenciáveis e seja $\varphi: M_{1} \rightarrow M_{2}$ uma aplicação diferenciável. Para cada $p \in M_{1}$ e cada $v \in T_{p} M_{1}$, escolha uma curva diferenciável $\alpha:(-\varepsilon, \varepsilon) \rightarrow M_{1}$ com $\alpha(0)=p, \alpha^{\prime}(0)=v$. Faça $\beta=\varphi \circ \alpha$. A aplicação $d \varphi_{p}: T_{p} M_{1} \rightarrow T_{\varphi(p)} M_{2}$ dada por $d \varphi_{p}(v)=\beta^{\prime}(0)$ é uma aplicação linear que não depende da escolha de $\alpha$.

Definição 1.4. A aplicação linear $d \varphi_{p}$ dada pela Proposição 1.1 é chamada diferencial de $f$ em $p$.

Definição 1.5. Sejam $M_{1}^{n}$ e $M_{2}^{n}$ variedades diferenciáveis. Uma aplicação $\varphi: M_{1} \rightarrow M_{2}$ é um difeomorfismo se ela é diferenciável, biunívoca, sobrejetiva e sua inversa $\varphi^{-1}$ é diferenciável. $\varphi$ é um difeomorfismo local em $p \in M$ se existem vizinhanças $U$ de $p$ e $V$ de $\varphi(p)$ tais que $\varphi: U \rightarrow V$ é um difeomorfismo.

A noção de difeomorfismo é a noção natural de equivalência entre variedades diferenciáveis.

Definição 1.6. Sejam $M^{m}$ e $N^{n}$ variedades diferenciáveis. Uma aplicação diferenciável $\varphi: M \rightarrow N$ é uma imersão se $d \varphi_{p}: T_{p} M \rightarrow T_{\varphi(p)} N$ é injetiva para todo $p \in M$. Se, além disto, $\varphi$ é um homeomorfismo sobre $\varphi(M) \subset N$, onde $\varphi(M)$ tem a topologia induzida por $N$, diz-se que $\varphi$ é um mergulho. Se $M \subset N$ e a inclusão $i: M \hookrightarrow N$ é um mergulho, diz-se que $M$ é uma subvariedade de $N$.

Note que se $\varphi: M^{m} \rightarrow N^{n}$ é uma imersão, então $m \leq n$; a diferença $n-m$ é chamada de codimensão da imersão $\varphi$. Pode-se mostrar que toda imersão é localmente um mergulho.

Definição 1.7. Seja $M$ uma variedade diferenciável. Dizemos que $M$ é orientável se $M$ admite uma estrutura diferenciável $\left\{\left(U_{\alpha}, X_{\alpha}\right)\right\}$ tal que, para todo par $\alpha, \beta$, com $X_{\alpha}\left(U_{\alpha}\right) \cap X_{\beta}\left(U_{\beta}\right)=W \neq \emptyset$, a diferencial da mudança de coordenadas $X_{\beta} \circ X_{\alpha}^{-1}$ tem determinante positivo. Caso contrário, diz-se que $M$ é não-orientável. Se $M$ é orientável, a escolha de uma estrutura diferenciável que satisfaz a condição acima é chamada uma orientação de $M$. Duas estruturas diferenciáveis que satisfazem tal condição determinam a mesma orientação se a união delas ainda a satisfaz.

Pode-se verificar que se $M$ é orientável e conexa existem exatamente duas orientações distintas em $M$. Supondo $M_{1}$ e $M_{2}$ variedades diferenciáveis e $\varphi: M_{1} \rightarrow M_{2}$ um difeomorfismo, pode-se verificar também que $M_{1}$ é orientável se e somente se $M_{2}$ é orientável. Se, além disto, $M_{1}$ e $M_{2}$ são conexas e 
estão orientadas, $\varphi$ induz uma orientação em $M_{2}$ que pode ou não coincidir com a orientação inicial de $M_{2}$. No primeiro caso, diz-se que $\varphi$ preserva orientação e no segundo, que $\varphi$ reverte a orientação.

Definição 1.8. Seja $M^{n}$ uma variedade diferenciável com estrutura diferenciável $\left(U_{\alpha}, X_{\alpha}\right)$ e considere o conjunto

$$
T M=\left\{(p, v) \mid p \in M, v \in T_{p} M\right\}
$$

Indicaremos por $\left(u_{1}^{\alpha}, \cdots, u_{n}^{\alpha}\right)$ as coordenadas de $U_{\alpha}$ e por $\left\{\frac{\partial}{\partial u_{1}^{\alpha}}, \cdots, \frac{\partial}{\partial u_{n}^{\alpha}}\right\}$ as bases associadas nos espaços tangentes de $\varphi_{\alpha}\left(U_{\alpha}\right)$. Para cada $\alpha$, defina $\phi_{\alpha}: U_{\alpha} \times \mathbb{R}^{n} \rightarrow T M$, por

$$
\phi_{\alpha}\left(u_{1}^{\alpha}, \cdots, u_{n}^{\alpha}, x_{1}, \cdots, x_{n}\right)=\left(\phi_{\alpha}\left(u_{1}^{\alpha}, \cdots, u_{n}^{\alpha}\right), \sum_{i=1}^{n} x_{i} \frac{\partial}{\partial u_{i}^{\alpha}}\right)
$$

onde $\left(x_{1}, \cdots, x_{n}\right) \in \mathbb{R}^{n}$. Assim definida, $\left\{\left(U_{\alpha} \times \mathbb{R}^{n}, \phi_{\alpha}\right)\right\}$ dota de uma estrutura diferenciável a TM. Com tal estrutura, TM é uma variedade diferenciável de dimensão $2 n$ chamada fibrado tangente.

Definição 1.9. Um campo de vetores $\mathcal{X}$ em uma variedade diferenciável $M$ é uma correspondência que a cada ponto $p \in M$ associa um vetor $\mathcal{X}(p) \in T_{p} M$. Em termos de aplicações, $\mathcal{X}$ é uma aplicação de $M$ no fibrado tangente TM. O campo é diferenciável se a aplicação $\mathcal{X}: M \rightarrow T M$ é diferenciável. Considerando uma parametrização $X: U \subset \mathbb{R}^{n} \rightarrow M$ é possivel escrever

$$
\mathcal{X}(p)=\sum_{i=1}^{n} a_{i}(p) \frac{\partial}{\partial u_{i}}
$$

onde cada $a_{i}: U \rightarrow \mathbb{R}$ é uma função em $U$ e $\left\{\frac{\partial}{\partial u_{i}}\right\}_{i=1}^{n}$ é a base associada a X. Dizemos que $\mathcal{X} e ́$ diferenciável se e somente se as funções $a_{i}$ são diferenciáveis para alguma (e, por tanto, para qualquer) parametrização.

Denotaremos por $\mathfrak{X}(M)$ o conjunto de todos os campos de vetores diferenciáveis em $M$ e por $\mathcal{D}(M)$ o conjunto das funções diferenciáveis de $M$ em $\mathbb{R}$. Assim, a expressão do campo $\mathcal{X}$ em (1.3) sugere uma forma de interpretar um campo de vetores como uma aplicação $\mathcal{X}: \mathcal{D}(M) \rightarrow \mathcal{D}(M)$, definida por

$$
(\mathcal{X} f)(p)=\sum_{i=1}^{n} a_{i}(p) \frac{\partial f}{\partial u_{i}}(p)
$$

sendo uma espécie de derivada direcional. Dessa forma, se $\mathcal{X}$ e $\mathcal{Y}$ são campos de vetores diferenciáveis em $M$ e $f: M \rightarrow \mathbb{R}$ é uma função diferenciável, podemos considerar as funções $\mathcal{X}(\mathcal{Y} f)$ e $\mathcal{Y}(\mathcal{X} f)$ e então definir o colchete, denotado por $[\mathcal{X}, \mathcal{Y}]$, pelo campo de vetores $\mathcal{X} \mathcal{Y}-\mathcal{Y} \mathcal{X}$ onde

$$
(\mathcal{X Y}-\mathcal{Y} \mathcal{X}) f=\mathcal{X}(\mathcal{Y} f)-\mathcal{Y}(\mathcal{X} f)
$$

É possível mostrar que para $\mathcal{X}, \mathcal{Y}, \mathcal{Z} \in \mathfrak{X}(M), a, b \in \mathbb{R}$ e $f, g \in \mathcal{D}(M)$, esta operação entre campos satisfaz as seguintes propriedades

1. $[\mathcal{X}, \mathcal{Y}]=-[\mathcal{Y}, \mathcal{X}]($ anti-comutatividade $)$

2. $[a \mathcal{X}+b \mathcal{Y}, \mathcal{Z}]=a[\mathcal{X}, \mathcal{Z}]+b[\mathcal{Y}, \mathcal{Z}]$ e $[\mathcal{X}, a \mathcal{Y}+b \mathcal{Z}]=a[\mathcal{X}, \mathcal{Y}]+b[\mathcal{X}, \mathcal{Z}]$ (bilinearidade) 
3. $[[\mathcal{X}, \mathcal{Y}], \mathcal{Z}]+[[\mathcal{Y}, \mathcal{Z}], X]+[[\mathcal{Z}, \mathcal{X}], Y]=0$ (identidade de Jacobi),

4. $[f \mathcal{X}, g \mathcal{Y}]=f g[\mathcal{Y}, \mathcal{X}]+f \mathcal{X}(g) \mathcal{Y}-g \mathcal{Y}(f) \mathcal{X}$.

Definição 1.10. Uma métrica Riemanniana em uma variedade diferenciável $M$ é uma correspondência que associa a cada ponto $p$ de $M$ uma forma bilinear simétrica, positiva definida $g_{p}: T_{p} M \times T_{p} M \rightarrow \mathbb{R}$ que varia diferenciavelmente no seguinte sentido: $S e X: U \subset \mathbb{R}^{n} \rightarrow M$ é um sistema de coordenadas locais em torno de $p$ com base associada $\left\{\left(\frac{\partial}{\partial u_{i}}\right)\right\}_{i=1}^{n}$ e $q=X\left(u_{1}, \cdots, u_{n}\right)$ então a função $g_{q}\left(\frac{\partial}{\partial u_{i}}(q), \frac{\partial}{\partial u_{j}}(q)\right)=$ $g_{i j}\left(u_{1}, \cdots, u_{n}\right)$ é uma função diferenciável em $U$. As funções $g_{i j}$ são chamadas expressão da métrica Riemanniana no sistema de coordenadas $X: U \subset \mathbb{R}^{n} \rightarrow M$. Uma variedade diferenciável munida de uma métrica Riemanniana chama-se uma Variedade Riemanniana.

Definição 1.11. Seja $M^{n}$ uma variedade Riemanniana com métrica $g$. Definimos o fibrado tangente unitário de $M$, denotado por $U M$, como a subvariedade

$$
U M=\{(x, \xi) \mid x \in M, g(\xi, \xi)=1\} .
$$

O fibrado tangente unitário $U M$ é uma subvariedade de dimensão $(2 n-1)$ que pode ser mergulhado em $M \times S^{n-1}$.

Definição 1.12. Sejam $M, N$ duas variedades Riemannianas. Dada $f: M \rightarrow N$ uma imersão, dizemos que $f$ é uma imersão isométrica se para todo $p \in M$ e $u, v \in T_{p} M$ temos

$$
g_{p}(u, v)=\tilde{g}_{f(p)}\left(d f_{p}(u), d f_{p}(v)\right) .
$$

Onde g representa a métrica de $M$ e g̃ a métrica de $N$, em particular, se $f$ é um difeomorfismo, dizemos que $f$ é uma isometria.

Observação 1.1. Observe que se $M, N$ são variedades diferenciáveis, $f: M \rightarrow N$ é uma imersão e $N$ possui uma estrutura Riemanniana então $f$ induz uma estrutura Riemanniana em $M$ para cada $p \in M$ da seguinte forma

$$
g_{p}(u, v):=\tilde{g}_{f(p)}\left(d f_{p}(u), d f_{p}(v)\right), \quad u, v \in T_{p} M .
$$

Onde g representa a métrica de $M$ e g̃ a métrica de $N$. Decorre do dito acima, que dada uma imersão isométrica $f: M \rightarrow N$, ela é localmente um mergulho.

Definição 1.13. Sejam $M, N$ duas variedades Riemannianas com métricas g e g̃ respectivamente. Uma aplicação $f: M \rightarrow N$ é chamada conforme se existe uma função $\varphi \in \mathcal{D}(M)$, tal que para todo $p \in M e$ todo $u, v \in T_{p} M$ se tenha

$$
\tilde{g}_{p}\left(d f_{p} u, d f_{p} v\right)=e^{2 \varphi} g_{p}(u, v) .
$$

Nesse caso, dizemos que g e $\tilde{g}$ são conformes. No caso particular de $\varphi$ ser constante, dizemos que g e $\tilde{g}$ são homotéticas.

Exemplo 1.2. Consideremos o semi-espaço do $\mathbb{R}^{n}$ dado por

$$
\mathbb{H}^{n}=\left\{\left(x_{1}, \cdots, x_{n}\right) \in \mathbb{R}^{n} \mid x_{n}>0\right\}
$$

e introduza em $\mathbb{H}^{n}$ a métrica

$$
g_{i j}\left(x_{1}, \cdots, x_{n}\right)=\frac{1}{x_{n}^{2}} \delta_{i j},
$$


assim tal métrica é conforme à métrica usual de $\mathbb{R}$. O espaço $\mathbb{H}^{n}$ é chamado o espaço Hiperbólico de dimensão $n$.

Já que o conteúdo principal do presente trabalho versa sobre subvariedades bidimensionais de $\mathbb{R}^{3}$, faremos um estudo das principais definições e propriedades para subvariedades em $\mathbb{R}^{n}$, sendo de utilidade o caso $n=3$ e as subvariedades de dimensão $m=2$, particularmente superfícies mínimas e superfícies Riemannianas. Nos seguintes capítulos, quando seja dita uma subvariedade, está subentendido que é uma subvariedade de $\mathbb{R}^{n}$.

\subsubsection{A métrica Riemanniana induzida às subvariedades de $\mathbb{R}^{n}$}

Em uma variedade diferenciável podem ser definidas muitas métricas diferentes, em nosso caso nos limitamos à chamada "métrica Riemanniana induzida" que é obtida do produto escalar Euclidiano do espaço envolvente.

Seja $M$ uma subvariedade de $\mathbb{R}^{n}$, o produto escalar Euclidiano em $\mathbb{R}^{n}$ é dado por

$$
\langle x, y\rangle:=\sum_{i=1}^{n} x_{i} y_{i}, \quad \text { para } x=\left(x_{1}, \cdots, x_{n}\right), y=\left(y_{1}, \cdots, y_{n}\right)
$$

Assim, $\langle\cdot, \cdot\rangle$ induz um produto escalar em cada subespaço $T_{p} M \subset \mathbb{R}^{n}$.

Definição 1.14. Seja $M \subset \mathbb{R}^{n}$ uma subvariedade e para cada $p \in M$, seja $g_{p}: T_{p} M \times T_{p} M \rightarrow \mathbb{R} o$ produto escalar dado por

$$
g_{p}(x, y):=\langle x, y\rangle, x, y \in T_{p} M
$$

A família $g=\left\{g_{p}\right\}_{p \in M}$ destes produtos escalares chama-se a métrica Riemanniana induzida em $M$.

\subsubsection{Gradiente, divergência e Laplaciano em subvariedades de $\mathbb{R}^{n}$}

Definição 1.15. Seja $M \subset \mathbb{R}^{n}$ uma subvariedade com sua métrica Riemanniana induzida e seja $f$ : $M \rightarrow R$ uma função suave em $M$ com valores reais. O gradiente de $f$ é o único campo vetorial

$$
\operatorname{grad} f: M \rightarrow \mathbb{R}^{n}
$$

em $M$ que a cada ponto $p \in M$ associa o vetor $\operatorname{grad} f(p) \in T_{p} M$ que satisfaz

$$
g_{p}(\operatorname{grad} f(p), v)=d f_{p}(v), \quad \forall v \in T_{p} M
$$

O seguinte teorema fornece uma representação em coordenadas locais para o gradiente na base canônica associada a um sistema de coordenadas, particularmente, mostra que o campo grad $f$ é suave.

Teorema 1.1. Seja $M^{m}$ uma subvariedade diferenciável e $(U, X)$ um sistema de coordenadas em $M$. Dada $f \in \mathcal{D}(M)$, denote a representação correspondente de $f$ por $\widehat{f}:=f \circ X^{-1}: X(U) \rightarrow \mathbb{R}$ e seja $\left(g^{i j}\right)$ a matriz inversa de $\left(g_{i j}\right)=\left(g_{p}\left(\frac{\partial}{\partial x_{i}}, \frac{\partial}{\partial x_{j}}\right)\right)$. Então no dominio $U$ temos

$$
\operatorname{grad} f=\sum_{i, j=1}^{m} g^{i j} \frac{\partial f}{\partial x_{i}} \cdot \frac{\partial}{\partial x_{j}}=\sum_{i, j=1}^{m} g^{i j}\left(\frac{\partial \widehat{f}}{\partial x_{i}} \circ X\right) \cdot \frac{\partial}{\partial x_{j}}
$$


Demonstração. Ver Apêndice A.1.1.

Definição 1.16. Seja $M^{m} \subset \mathbb{R}^{n}$ uma subvariedade diferenciável e $\mathcal{X} \in \mathfrak{X}(M)$. A divergência de $\mathcal{X}$ é a função $\operatorname{div}(\mathcal{X}) \in \mathcal{D}(M)$ definida por

$$
\operatorname{div}(\mathcal{X})(p)=\sum_{i=1}^{m}\left\langle v_{i}(\mathcal{X}), v_{i}\right\rangle, \quad p \in M
$$

onde $\left\{v_{1}, \cdots, v_{m}\right\}$ é uma base ortonormal de $T_{p} M$ e $v_{i}(\mathcal{X}) \in \mathbb{R}^{n}$ denota a derivada direcional da aplicação $\mathcal{X}: M \rightarrow \mathbb{R}^{n}$ na direção do vetor $v_{i}$.

Agora, vamos escrever a divergência de um campo vetorial em coordenadas locais, para tal propósito, vamos precisar de um Lema, cuja demonstração encontra-se no Apêndice A, Seção A.1.2.

Lema 1.1. Seja $F:(-\varepsilon, \varepsilon) \rightarrow G L(n, \mathbb{C})$ uma curva diferenciável no grupo das matrizes invertíveis. Então

$$
\operatorname{Tr}\left(F^{-1}(s) \circ F^{\prime}(s)\right)=\frac{d}{d s} \ln (\operatorname{det} F(s)) .
$$

Teorema 1.2. Seja $M^{m}$ uma subvariedade diferenciável com parametrização $X: U \rightarrow M$ e $\mathcal{X} \in \mathfrak{X}(M)$. Suponha que $\mathcal{X}$ tenha a representação $\mathcal{X}=\sum_{i=1}^{m} \xi_{i} \frac{\partial}{\partial u_{i}}$. Além disso, seja $\theta=\operatorname{det}\left(g_{i j}(p)\right)$, para cada $p \in M$. Então no dominio $U$, temos

$$
\operatorname{div}(\mathcal{X})=\frac{1}{\sqrt{\theta}} \sum_{i=1}^{m} \frac{\partial}{\partial u_{i}}\left(\xi_{i} \sqrt{\theta}\right)
$$

Demonstração. Seja $\left\{\frac{\partial}{\partial u_{1}}, \cdots, \frac{\partial}{\partial u_{m}}\right\}$ a base canônica associada a $p$ em $M$ induzida pela parametrização $X$ e seja $\left(v_{1}, \cdots, v_{m}\right)$ uma base ortonormal qualquer em $T_{p} M$. Então $v_{i}=\sum_{j=1}^{m} A_{i j} \frac{\partial}{\partial u_{j}}$ para uma matriz $A=\left(A_{i j}\right)$. Além disso, seja $E:=\left(\delta_{i j}\right)$ a matriz identidade e seja $G:=\left(g_{i j}(p)\right)$. As matrizes $G$ e $A$ são relacionadas da seguinte forma

$$
E=\left(g_{p}\left(a_{i}, a_{j}\right)\right)=\left(\sum_{k, l=1}^{n} A_{i k} g_{k l} A_{j l}\right)=A \circ G \circ A^{t}, \quad \text { e também } \quad G^{-1}=A^{t} \circ A .
$$

Em particular,

$$
g^{i j}=\left(A^{t} A\right)_{i j}
$$

Para a divergência obtemos, pela definição:

$$
\begin{aligned}
\operatorname{div}(\mathcal{X})=\sum_{i=1}^{m}\left\langle v_{i}(\mathcal{X}), v_{i}\right\rangle & =\sum_{i, k, l=1}^{m} A_{i k}\left\langle\frac{\partial}{\partial u_{k}}(\mathcal{X}), \frac{\partial}{\partial u_{l}}\right\rangle A_{i l} \\
& =\sum_{k, l=1}^{m}\left(\sum_{i=1}^{m} A_{i k} A_{i l}\right)\left\langle\frac{\partial}{\partial u_{k}}(\mathcal{X}), \frac{\partial}{\partial u_{l}}\right\rangle \\
& =\sum_{k, l=1}^{m}\left(A^{t} A\right)_{k l}\left\langle\frac{\partial}{\partial u_{k}}(\mathcal{X}), \frac{\partial}{\partial u_{l}}\right\rangle \\
& \stackrel{(1.4)}{=} \sum_{k, l=1}^{m} g^{k l}\left\langle\frac{\partial}{\partial u_{k}}(\mathcal{X}), \frac{\partial}{\partial u_{l}}\right\rangle .
\end{aligned}
$$


Agora substituímos $\mathcal{X}$ pela representação $\mathcal{X}=\sum_{i=1}^{m} \xi_{i} \frac{\partial}{\partial u_{i}}$ e aplicamos as regras para derivada direcional. Além disso, no seguinte calculo vamos usar o fato das derivadas direcionais e vetores de uma base canônica comutarem segundo a fórmula

$$
\left[\frac{\partial}{\partial u_{k}}, \frac{\partial}{\partial u_{j}}\right]=\frac{\partial}{\partial u_{k}} \frac{\partial}{\partial u_{j}}-\frac{\partial}{\partial u_{j}} \frac{\partial}{\partial u_{k}}=0
$$

Desta forma, obtemos

$$
\begin{aligned}
\operatorname{div}(\mathcal{X}) & =\sum_{j, k, l=1}^{m} g^{k l}\left(\left\langle\frac{\partial}{\partial u_{k}}\left(\xi_{j}\right) \cdot \frac{\partial}{\partial u_{j}}, \frac{\partial}{\partial u_{l}}\right\rangle+\xi_{j}\left\langle\frac{\partial}{\partial u_{k}}\left(\frac{\partial}{\partial u_{j}}\right), \frac{\partial}{\partial u_{l}}\right\rangle\right) \\
& =\sum_{j, k, l=1}^{m}\left[\frac{\partial}{\partial x_{k}}\left(\xi_{j}\right) g^{k l} g_{j l}+\xi_{j} g^{k l}\left\langle\frac{\partial}{\partial u_{j}}\left(\frac{\partial}{\partial u_{k}}\right), \frac{\partial}{\partial u_{l}}\right\rangle\right] \\
& =\sum_{j=1}^{m} \frac{\partial}{\partial u_{j}}\left(\xi_{j}\right)+\sum_{j=1}^{m} \xi_{j} \sum_{k, l=1}^{m} g^{k l}\left\langle\frac{\partial}{\partial u_{j}}\left(\frac{\partial}{\partial u_{k}}\right), \frac{\partial}{\partial u_{l}}\right\rangle,
\end{aligned}
$$

onde na penúltima linha no segundo termo, trocamos os índices $j$ e $k$ segundo nossa observação acima. Continuamos transformando o segundo termo. Para isso, separamos ele em duas partes iguais e na segunda parte renomeamos os índices $k$ e $l$. Além disso, usamos a simetria da métrica, a regra da derivada do produto escalar de aplicações com valores vetoriais e o lema anterior, assim

$$
\begin{aligned}
\sum_{k, l=1}^{m} g^{k l}\left\langle\frac{\partial}{\partial u_{j}}\left(\frac{\partial}{\partial u_{k}}\right), \frac{\partial}{\partial u_{l}}\right\rangle & =\frac{1}{2} \sum_{k, l=1}^{m} g^{k l}\left\langle\frac{\partial}{\partial u_{j}}\left(\frac{\partial}{\partial u_{k}}\right), \frac{\partial}{\partial u_{l}}\right\rangle+\frac{1}{2} \sum_{l, k=1}^{m} g^{l k}\left\langle\frac{\partial}{\partial u_{j}}\left(\frac{\partial}{\partial u_{l}}\right), \frac{\partial}{\partial u_{k}}\right\rangle \\
& =\frac{1}{2} \sum_{k, l=1}^{m} g^{k l}\left\langle\frac{\partial}{\partial u_{j}}\left(\frac{\partial}{\partial u_{k}}\right), \frac{\partial}{\partial u_{l}}\right\rangle+\frac{1}{2} \sum_{l, k=1}^{m} g^{k l}\left\langle\frac{\partial}{\partial u_{k}}\left(\frac{\partial}{\partial u_{j}}\right), \frac{\partial}{\partial u_{l}}\right\rangle \\
& =\frac{1}{2} \sum_{k, l=1}^{m} g^{k l} \frac{\partial}{\partial u_{j}}\left(\left\langle\frac{\partial}{\partial u_{k}}, \frac{\partial}{\partial u_{l}}\right\rangle\right) \\
& =\frac{1}{2} \sum_{k, l=1}^{m} g^{k l} \frac{\partial}{\partial u_{j}}\left(g_{k l}\right) \\
& =\frac{1}{2} \operatorname{Tr}\left(G^{-1} \cdot \frac{\partial}{\partial u_{j}}(G)\right) \\
L e & =\frac{1}{2} \frac{1}{2} \frac{\partial}{\partial u_{j}}(\ln (\operatorname{det} G)) \\
& =\frac{1}{2} \frac{\partial}{\partial u_{j}}(\ln (\theta)) \\
& =\frac{\partial}{\partial u_{j}}(\ln (\sqrt{\theta}) \\
& =\frac{1}{\sqrt{\theta}} \frac{\partial}{\partial u_{j}}(\sqrt{\theta}) .
\end{aligned}
$$

Usando a equação (1.5) obtemos

$$
\operatorname{div}(\mathcal{X})=\sum_{j=1}^{m}\left[\frac{\partial}{\partial u_{j}}\left(\xi_{j}\right)+\frac{1}{\sqrt{\theta}} \cdot \xi_{i} \frac{\partial}{\partial x_{j}}(\sqrt{\theta})\right]=\frac{1}{\sqrt{\theta}} \sum_{i=1}^{m} \frac{\partial}{\partial u_{i}}\left(\xi^{i} \sqrt{\theta}\right)
$$


Finalmente, definimos o Laplaciano para funções definidas em subvariedades.

Definição 1.17. Seja $M^{m} \subset \mathbb{R}^{n}$ uma subvariedade diferenciável com métrica g e $f \in \mathcal{D}(M)$. Definimos o operador de Laplace $\Delta^{g}$ por

$$
\begin{aligned}
\Delta^{g}: \mathcal{D} & \rightarrow \mathcal{D} \\
f & \mapsto \Delta^{g}(f)=\operatorname{div}(\operatorname{grad}(f)) .
\end{aligned}
$$

Quando seja usada uma única métrica, simplesmente denotaremos o operador Laplaciano por $\Delta$. Assim, como foi determinada a divergência em coordenadas locais, temos a seguinte representação para o operador Laplaciano.

Teorema 1.3. Seja $M^{m}$ uma subvariedade diferenciável com parametrização $X: U \rightarrow M$ e $f \in \mathcal{D}(M)$. Então, com as notações do Teorema 1.2, temos

$$
\Delta f=\frac{1}{\sqrt{\theta}} \sum_{i, j=1}^{m} \frac{\partial}{\partial u_{j}}\left(\sqrt{\theta} g^{i j} \frac{\partial f}{\partial u_{i}}\right) .
$$

Demonstração. Da mesma definição do operador Laplaciano $\Delta$ e dos Teoremas 1.1 e 1.2 segue que no domínio $U$, temos

$$
\begin{aligned}
\Delta\left(\left.f\right|_{U}\right) & =\operatorname{div}\left[\sum_{j=1}^{m}\left(\sum_{i=1}^{m} g^{i j} \frac{\partial f}{\partial u_{i}}\right) \frac{\partial}{\partial u_{j}}\right] \\
& =\frac{1}{\sqrt{\theta}} \sum_{j=1}^{m} \frac{\partial}{\partial u_{j}}\left(\sqrt{\theta} \sum_{i=1}^{m} g^{i j} \frac{\partial f}{\partial u_{i}}\right) \\
& =\frac{1}{\sqrt{\theta}} \sum_{i, j=1}^{m} \frac{\partial}{\partial u_{j}}\left(\sqrt{\theta} g^{i j} \frac{\partial f}{\partial u_{i}}\right) .
\end{aligned}
$$

Exemplo 1.3. O operador de Laplace em $S^{2}$. Seja a métrica g induzida pela imersão

$$
\begin{aligned}
T:(0, \pi) \times(0,2 \pi) & \rightarrow S^{2} \\
(\varphi, \phi) \quad & \mapsto T(\varphi, \phi)=(\sin \varphi \cos \phi, \sin \varphi \sin \phi, \cos \varphi),
\end{aligned}
$$

então, temos

$$
g_{11}=\left\langle T_{\varphi}, T_{\varphi}\right\rangle=1 \quad, \quad g_{12}=g_{21}=0 \quad, \quad g_{22}=\left\langle T_{\phi}, T_{\phi}\right\rangle=\operatorname{sen}^{2} \varphi
$$

$e$

$$
\theta=\left|\begin{array}{cc}
1 & 0 \\
0 & \sin ^{2} \varphi
\end{array}\right|=\sin ^{2} \varphi
$$

assim,

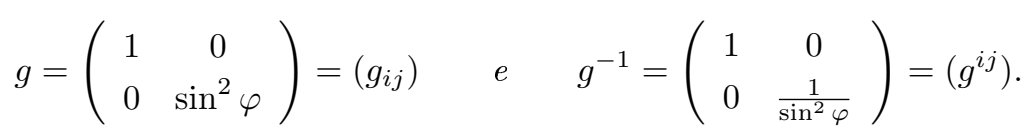


Logo, temos

$$
\begin{aligned}
\Delta & =-\frac{1}{\theta^{1 / 2}}\left(\frac{\partial}{\partial \varphi} \theta^{1 / 2} \frac{\partial}{\partial \varphi}+\frac{\partial}{\partial \phi} \frac{1}{\sin ^{2} \varphi} \theta^{1 / 2} \frac{\partial}{\partial \phi}\right) \\
& =-\frac{1}{\sin \varphi}\left[\frac{\partial}{\partial \varphi}\left(\sin \varphi \frac{\partial}{\partial \varphi}\right)+\frac{\partial}{\partial \phi} \frac{1}{\sin \varphi} \frac{\partial}{\partial \phi}\right] \\
& =-\frac{1}{\sin \varphi}\left[\frac{\partial}{\partial \varphi}\left(\sin \varphi \frac{\partial}{\partial \varphi}\right)\right]-\frac{1}{\sin ^{2} \varphi} \frac{\partial}{\partial \phi^{2}}
\end{aligned}
$$

Exemplo 1.4. (Operador de Laplace para métricas conformes)

Seja $M^{m}$ uma variedade Riemanniana com duas métricas conformes $g$ e $\tilde{g}$, então existe $\varphi \in \mathcal{D}(M)$ tal que

$$
\tilde{g}=e^{2 \varphi} g
$$

assim, obtemos que $\tilde{g_{i j}}=e^{2 \varphi} g_{i j}$ e consequentemente que $\tilde{g}^{i j}=e^{-2 \varphi} g^{i j}$. É claro que da equação (1.6) temos que $\operatorname{det} \tilde{g}=e^{2 m \varphi} \operatorname{det} g$, então, definindo $\theta:=\operatorname{det} g$ obtemos $\operatorname{det} \tilde{g}=e^{2 m \varphi} \theta=: \tilde{\theta}$. Agora, fazendo uso da fórmula do Laplaciano em coordenadas locais dada no Teorema 1.3 temos

$$
\begin{aligned}
\Delta^{\tilde{g}} & =\frac{1}{\sqrt{\tilde{\theta}}} \sum_{i, j=1}^{m} \frac{\partial}{\partial u_{j}}\left(\sqrt{\tilde{\theta}} \tilde{g}^{i j} \frac{\partial}{\partial u_{i}}\right) \\
& =\frac{1}{e^{m \varphi} \sqrt{\theta}} \sum_{i, j=1}^{m} \frac{\partial}{\partial u_{j}}\left(e^{\left.m \varphi \sqrt{\theta} e^{-2 \varphi} g^{i j} \frac{\partial}{\partial u_{i}}\right)}\right. \\
& =\frac{1}{e^{m \varphi} \sqrt{\theta}} \sum_{i, j=1}^{m} \frac{\partial}{\partial u_{j}}\left(e^{(m-2) \varphi} \sqrt{\theta} g^{i j} \frac{\partial}{\partial u_{i}}\right) \\
& =\frac{1}{e^{m \varphi} \sqrt{\theta}} \sum_{i, j=1}^{m}\left[\left(e^{(m-2) \varphi}(m-2) \frac{\partial \varphi}{\partial u_{j}}\right) \sqrt{\theta} g^{i j} \frac{\partial}{\partial u_{i}}+e^{(m-2) \varphi} \frac{\partial}{\partial u_{j}}\left(\sqrt{\theta} g^{i j} \frac{\partial}{\partial u_{i}}\right)\right] \\
& =(m-2) e^{-2 \varphi} \sum_{i, j=1}^{m} g^{i j} \frac{\partial \varphi}{\partial u_{j}} \frac{\partial}{\partial u_{i}}+\frac{e^{-2 \varphi}}{\sqrt{\theta}} \sum_{i, j=1}^{m} \frac{\partial}{\partial u_{j}}\left(\sqrt{\theta} g^{i j} \frac{\partial f}{\partial u_{i}}\right) \\
& =(m-2) e^{-2 \varphi} \sum_{i, j=1}^{m} g^{i j} \frac{\partial \varphi}{\partial u_{j}} \frac{\partial}{\partial u_{i}}+e^{-2 \varphi} \Delta^{g} .
\end{aligned}
$$

Já que estamos sobre superfícies (subvariedades 2-dimensionais) temos a seguinte relação entre os Laplacianos das métricas conformes

$$
\Delta^{\tilde{g}}=e^{-2 \varphi} \Delta^{g} .
$$

A continuação serão citadas as regras do produto para o gradiente, divergência e Laplaciano em uma subvariedade. As demonstrações encontram-se no Apêndice A, Seção A.1.3.

Teorema 1.4. Seja $M$ uma subvariedade, sejam $f, h \in \mathcal{D}(M)$ e $\mathcal{X} \in \mathfrak{X}(M)$. Então

1. $\operatorname{grad}(f \cdot h)=f \cdot \operatorname{grad}(h)+h \cdot \operatorname{grad}(f)$.

2. $\operatorname{div}(f \cdot \mathcal{X})=f \cdot \operatorname{div}(\mathcal{X})+\mathcal{X}(f)$.

3. $\Delta(f \cdot h)=f \cdot \Delta h+h \cdot \Delta f+2\langle\operatorname{grad} f, \operatorname{grad} h\rangle$. 
Definição 1.18. Uma conexão afim $\nabla$ sobre uma variedade diferenciável $M$ é uma aplicação

$$
\begin{aligned}
\nabla: \mathfrak{X}(M) \times \mathfrak{X}(M) & \rightarrow \mathfrak{X}(M) \\
(\mathcal{X}, \mathcal{Y}) & \mapsto \nabla(\mathcal{X}, \mathcal{Y}) \stackrel{\text { not. }}{=} \nabla_{\mathcal{X Y}},
\end{aligned}
$$

satisfazendo as seguintes propriedades

i. $\nabla_{f \mathcal{X}+g \mathcal{Y}} \mathcal{Z}=f \nabla_{\mathcal{X}} \mathcal{Z}+g \nabla_{\mathcal{Y}} \mathcal{Z}$

ii. $\nabla_{\mathcal{X}}(\mathcal{Y}+\mathcal{Z})=\nabla_{\mathcal{X}} \mathcal{Y}+\nabla_{\mathcal{X}} \mathcal{Z}$

iii. $\nabla_{\mathcal{X}}(f \mathcal{Y})=f \nabla_{\mathcal{X}} \mathcal{Y}+\mathcal{X}(f) \mathcal{Y}$

onde $\mathcal{X}, \mathcal{Y}, \mathcal{Z} \in \mathfrak{X}(M)$ e $f, g \in \mathcal{D}(M)$.

Definição 1.19. Dada uma variedade Riemanniana $M^{m}$ e $p \in M$, existe uma vizinhança $U \subset M$ de $p$ e $m$ campos ortonormais $E_{1} \cdots E_{m} \in \mathfrak{X}(U)$ tais que em $p, \nabla_{E_{i}} E_{j}(p)=0$. A família $\left\{E_{i}\right\}_{i=1}^{m}$ é chamada um referencial geodésico (local) em $p$.

Observação 1.2. Das duas definições anteriores, o operador de Laplace na Definição 1.17 é equivalente $a$

$$
\Delta(f(p))=\sum_{i=1}^{m} E_{i}\left(E_{i}(f)\right)(p), \forall p \in M
$$

\subsection{Superfícies, formas quadráticas e representação de Weierstrass}

\subsubsection{Superfícies regulares}

Definição 1.20. Um subconjunto $S \subset \mathbb{R}^{3}$ é uma superfície regular se, para cada $p \in S$ existe uma vizinhança $V$ de $p$ em $\mathbb{R}^{3}$ e uma aplicação $\varphi: U \rightarrow V \cap S$ de um aberto $U$ de $\mathbb{R}^{2}$ sobre $V \cap S \subset \mathbb{R}^{3}$ tal que

1. $\varphi$ é diferenciável.

Isto é, se escrevemos $\varphi(u, v)=(x(u, v), y(u, v), z(u, v)) \in U$, as funções $x(u, v), y(u, v), z(u, v)$ têm derivadas parciais continuas de todas as ordens em $U$.

2. $\varphi$ é injetiva.

3. Para todo $q \in U,\left\{\frac{\partial \varphi}{\partial u}, \frac{\partial \varphi}{\partial v}\right\}$ são linearmente independentes, ou seja, as derivadas parciais $\frac{\partial \varphi}{\partial u}(q) e$ $\frac{\partial \varphi}{\partial v}(q)$ são tais que o produto vetorial $\frac{\partial \varphi}{\partial u}(q) \times \frac{\partial \varphi}{\partial v}(q) \neq 0$.

Quando dizer superfície é comum identificar a parametrização $\phi$ quanto sua imagem, $S=\varphi(U)$, sendo indistintamente usado no decorrer do trabalho.

Definição 1.21. Dizemos que uma superfície $S$ é orientável se pode ser coberta com uma familia de vizinhanças coordenadas de modo que se um ponto $p \in S$ estar em duas vizinhanças dessa família, o Jacobiano da mudança de parâmetros em p seja positivo.

Entendemos por vetor tangente a $S$ em um ponto $p \in S$, o vetor $\alpha^{\prime}(t)$ de uma curva parametrizada diferenciável $\alpha:(-\epsilon, \epsilon) \rightarrow S \operatorname{com} \alpha(0)=p$. Seja $S \subset \mathbb{R}^{3}$ uma superfície regular, $\varphi: U \subset \mathbb{R}^{2} \rightarrow V \cap S$ 
uma parametrização e um ponto $q \in U$. O conjunto de vetores tangentes a $S$ em $p=\varphi(q)$ é um subespaço 2 - dimensional chamado plano tangente de $S$ em p denotado por $T_{p} S$ é independente da parametrização $\varphi$ escolhida.

Já que $\varphi_{u} \times \varphi_{v}$ é ortogonal ao plano tangente $T_{p} S$ no ponto $p$, representamos o vetor normal unitário em $p$ por

$$
N(p)=\frac{\varphi_{u}(q) \times \varphi_{v}(q)}{\left|\varphi_{u}(q) \times \varphi_{v}(q)\right|}
$$

chamado de vetor normal unitário de $\varphi$ em $q$, sendo $p=\varphi(q)$. Dessa forma, para $p$ fixado, a equação de $T_{p} S$ é dada por

$$
(x, y, z)-\varphi(q) N(p)=0 .
$$

\subsubsection{Formas fundamentais}

Vamos considerar uma superfície regular $S \subset \mathbb{R}^{3}$. Em cada plano tangente $T_{p} S$ com $p \in S$ é induzido um produto interno $\langle\cdot, \cdot\rangle_{p}$, que, por sua vez é uma forma bilinear simétrica, logo tem associada uma forma quadrática $I_{p}$. Definimos o produto interno $\langle\cdot, \cdot\rangle_{p}$ e a forma quadrática $I_{p}$, respectivamente, como

$$
\begin{aligned}
\langle\cdot, \cdot\rangle_{p}: T_{p} S \times T_{p} S & \rightarrow \mathbb{R} \\
(u, v) & \mapsto\langle u, v\rangle_{p}
\end{aligned}
$$

e

$$
\begin{aligned}
I_{p}: T_{p} S & \rightarrow \mathbb{R} \\
w & \mapsto I_{p}(w)=|w|^{2} .
\end{aligned}
$$

A forma quadrática $I_{p}$ é chamada primeira forma fundamental da superfície regular $S$ em $p$.

Supondo que $X: U \subset \mathbb{R}^{2} \rightarrow \mathbb{R}^{3}$ é uma superfície regular, sendo que $\left\{X_{u}, X_{v}\right\}$ é base de $T_{p} S$, para $a X_{u}+b X_{v}$ com $a, b \in \mathbb{R}$, podemos escrever

$$
\begin{aligned}
I_{p}\left(a X_{u}+b X_{v}\right) & =\left|a X_{u}+b X_{v}\right|^{2} \\
& =a^{2}\left\langle X_{u}, X_{u}\right\rangle+2 a b\left\langle X_{u}, X_{v}\right\rangle+b^{2}\left\langle X_{v}, X_{v}\right\rangle
\end{aligned}
$$

e obtemos as seguintes funções

$$
\begin{aligned}
g_{11} & =\left\langle X_{u}, X_{u}\right\rangle:=E \\
g_{12}=g_{21} & =\left\langle X_{u}, X_{v}\right\rangle:=F \\
g_{22} & =\left\langle X_{v}, X_{v}\right\rangle:=G
\end{aligned}
$$

Chamamos às funções $\left\{g_{i j}\right\}$ de coeficientes da primeira forma fundamental. Explicitamente, a primeira forma fundamental pode-se exprimir como

$$
I=E d u^{2}+2 F d u d v+G d v^{2}
$$

Agora, considerando a superfície regular $S$ orientada parametrizada por $X: U \subset \mathbb{R}^{2} \rightarrow S$, definimos 
a aplicação normal de Gauss em $p \in S$ como

$$
\begin{aligned}
N: U \subset \mathbb{R}^{2} & \rightarrow \mathbb{R} \\
(u, v) & \mapsto N(u, v)=\frac{X_{u} \times X_{v}}{\left|X_{u} \times X_{v}\right|},
\end{aligned}
$$

a qual associa a cada ponto de $S$ sua imagem normal em $S^{2}$. Sabemos que $d N_{p}: T_{p} S \rightarrow T_{p} S$ é uma aplicação linear autoadjunta, o que permite associar-lhe uma forma quadrática $Q_{p}$ definida como

$$
\begin{aligned}
Q_{p}: T_{p} S \times T_{p} S & \rightarrow \mathbb{R} \\
(u, v) & \mapsto Q(u, v)=\left\langle d N_{p} u, v\right\rangle .
\end{aligned}
$$

Associamos a $Q_{p}$ a forma quadrática $I I_{p}$ definida como

$$
\begin{aligned}
I I_{p}: T_{p} S & \rightarrow \mathbb{R} \\
u & \mapsto I I_{p}(u)=-Q(u, u)=-\left\langle d N_{p} u, u\right\rangle .
\end{aligned}
$$

A forma quadrática $I I_{p}$ é chamadasegunda forma fundamental da superfície regular $S$ em $p$.

Analogamente ao caso da primeira forma fundamental, para $a X_{u}+b X_{v}$ com $a, b \in \mathbb{R}$, podemos escrever

$$
\begin{aligned}
I I_{p}\left(a X_{u}+b X_{v}\right) & =-\left\langle d N_{p}\left(a X_{u}+b X_{v}\right), a X_{u}+b X_{v}\right\rangle \\
& =-a^{2}\left\langle d N_{p}\left(X_{u}\right), X_{u}\right\rangle-2 a b\left\langle d N_{p}\left(X_{u}\right), X_{v}\right\rangle-b^{2}\left\langle d N_{p}\left(X_{v}\right), X_{v}\right\rangle \\
& =a^{2}\left\langle N, X_{u u}\right\rangle+2 a b\left\langle N, X_{u v}\right\rangle+b^{2}\left\langle N, X_{v v}\right\rangle
\end{aligned}
$$

e obtemos as seguintes funções

$$
\begin{aligned}
e_{11} & =\left\langle N, X_{u u}\right\rangle=-\left\langle N_{u}, X_{u}\right\rangle:=e \\
e_{12}=e_{21} & =\left\langle N, X_{u v}\right\rangle=-\left\langle N_{u}, X_{v}\right\rangle=-\left\langle N_{v}, X_{u}\right\rangle:=f \\
e_{22} & =\left\langle N, X_{v v}\right\rangle=-\left\langle N_{v}, X_{v}\right\rangle:=g
\end{aligned}
$$

Chamamos às funções $\left\{e_{i j}\right\}$ de coeficientes da segunda forma fundamental. Explicitamente, a segunda forma fundamental pode-se exprimir como

$$
I I=e d u^{2}+2 f d u d v+g d v^{2} .
$$

Definição 1.22. Seja $X: U \subset \mathbb{R}^{2} \rightarrow \mathbb{R}^{3}$ uma superfície regular, definimos a curvatura Gaussiana como

$$
K=\frac{e_{11} e_{22}-e_{12}^{2}}{g_{11} g_{22}-g_{12}^{2}}
$$

e se for uma superfície orientada, definimos a curvatura média como

$$
H=\frac{1}{2} \frac{e_{11} g_{22}-2 e_{12} g_{12}+e_{22} g_{22}}{g_{11} g_{22}-g_{12}^{2}} .
$$

Definição 1.23. Dizemos que uma superfície orientada é mínima se sua curvatura média é identicamente nula. 
Finalmente, é possível definir uma outra forma bilinear simétrica dada por

$$
\begin{aligned}
P_{p}: T_{p} S \times T_{p} S & \rightarrow \mathbb{R} \\
(u, v) & \mapsto P_{p}(u, v)=\left\langle d N_{p} u, d N_{p} v\right\rangle,
\end{aligned}
$$

associando-lhe a forma quadrática $I I I_{p}$ definida como

$$
\begin{aligned}
I I I_{p}: T_{p} S & \rightarrow \mathbb{R} \\
u & \mapsto I I I_{p}(u)=\left\langle d N_{p} u, d N_{p} u\right\rangle .
\end{aligned}
$$

A forma quadrática $I I I_{p}$ é chamada terceira forma fundamental da Superfície $S$ em $p$. Assim, das equacoes (1.7) e (1.8), a terceira forma fundamental pode-se exprimir como

$$
I I I=(2 H e-K E) d u^{2}+2(2 H f-K F) d u d v+(2 H g-K G) d v^{2} .
$$

De agora em diante, omitiremos o índice $p$, subentendido que as formas fundamentais estão definidas em $p$. Se não ter confusão, será usado $I I I(u, v)$ para fazer referência à forma bilinear $P$ da qual $I I I$ provém. Assim também, para $I I(u, v)$, teremos considerado $I I(u, v)=-\left\langle d N_{p} u, v\right\rangle$, ou seja $I I(u, v)=$ $-Q(u, v)$. Analogamente para $I$.

Observação 1.3 (Relação entre as Formas Fundamentais). Dada uma superfície orientada $S$ e um ponto $p \in S$, existe uma base ortonormal $\left\{e_{1}, e_{2}\right\}$ do $T_{p} S$ de direções principais, então: $d N_{p}\left(e_{1}\right)=$ $-k_{1} e_{1}, d N_{p}\left(e_{2}\right)=-k_{2} e_{2}$ onde $k_{1}, k_{2}$ são as curvaturas principais de $S$ em p. Elas provém da equação

$$
k^{2}-2 H k+K=0,
$$

onde $H$ e K são a curvatura média e Gaussiana respectivamente. Tal equação é fornecida pelo polinômio característico

$$
p(k)=\operatorname{det}\left(d N_{p}+k I d\right)=0,
$$

logo, usando o Teorema de Cayley-Hamilton temos

$$
\left(d N_{p}\right)^{2}-2 H d N_{p}+K I d=0 .
$$

Agora, $\forall u \in T_{p} S$ temos

$$
\begin{array}{r}
\left\langle\left(d N_{p}\right)^{2} u, u\right\rangle-2 H\left\langle d N_{p} u, u\right\rangle+K\langle u, u\rangle=0 \\
\left\langle d N_{p} u, d N_{p} u\right\rangle-2 H\left\langle d N_{p} u, u\right\rangle+K\langle u, u\rangle=0 \\
I I I(u)-2 H I I(u)+k I(u)=0
\end{array}
$$

assim, temos a identidade

$$
I I I-2 H I I+K I=0 .
$$

Consequentemente, da equação (1.10) temos as seguintes funções

$$
\begin{aligned}
h_{11} & =-2 H e_{11}-K g_{11} \\
h_{12}=h_{21} & =-2 H e_{12}-K g_{12} \\
h_{22} & =-2 H e_{22}-K g_{22}
\end{aligned}
$$


Chamamos às funções $\left\{h_{i j}\right\}$ de coeficientes da terceira forma fundamental.

De modo geral, seja $S$ uma superície orientada. As formas quadráticas $A=\tilde{L} d u^{2}+2 \tilde{M} d u d v+\tilde{N} d v^{2}$ e $B=L d u^{2}+2 M d u d v+N d v^{2}$ sobre $S$ fornecem o par $(A, B)$ se $A$ é não degenerada. É conveniente usar a terminología usual quando $A=I$ e $B=I I$ são as formas fundamentais.

Definimos a curvatura média e a curvatura extrínseca $\mathrm{H}$ e $\mathrm{K}$, respectivamente, do par $(A, B)$ por

$$
\begin{aligned}
\mathrm{H}=\mathrm{H}(A, B) & =\frac{\tilde{L} N-2 \tilde{M} M+\tilde{N} L}{2\left(\tilde{L} \tilde{N}-\tilde{M}^{2}\right)}, \\
\mathrm{K}=\mathrm{K}(A, B) & =\frac{L N-M^{2}}{\tilde{L} \tilde{N}-\tilde{M}^{2}} .
\end{aligned}
$$

No caso particular de ter as formas quadráticas $C=I I I$ e $B=I I$, temos o par $(C, B)=(I I I, I I)$, e usando as expressões em (1.9) e (1.8) temos,

$$
\begin{gathered}
I I I=\underbrace{(2 H e-K E)}_{\tilde{L}} d u^{2}+2 \underbrace{(2 H f-K F)}_{\tilde{M}} d u d v+\underbrace{(2 H g-K G)}_{\tilde{N}} d v^{2} \\
I I=\underbrace{e}_{L} d u^{2}+2 \underbrace{f}_{M} d u d v+\underbrace{g}_{N} d v^{2}
\end{gathered}
$$

daí,

$$
\begin{aligned}
\mathrm{H}(I I I, I I) & =\frac{(2 H e-K E) g-2(2 H f-K F) f+(2 H g-K G) e}{(2 H e-K E)(2 H g-K G)-(2 H f-K F)^{2}} \\
& =\frac{2 H\left(2 e g-2 f^{2}\right)-K(E g+2 f F-G e)}{4 H^{2} e g-2 H e K G-2 K E H g+K^{2} E G-4 H^{2} f^{2}+4 H f K F-K^{2} F^{2}} \\
& =\frac{4 H\left(e g-f^{2}\right)-K \cdot 2\left(E G-F^{2}\right) H}{4 H^{2}\left(e g-f^{2}\right)-2 H K(e G-2 f F+E g)+K^{2}\left(E G-F^{2}\right)} \\
& =\frac{4 H\left(e g-f^{2}\right)-\left(\frac{e g-f^{2}}{E G-F^{2}}\right) 2\left(E G-F^{2}\right) H}{4 H^{2}\left(e g-f^{2}\right)-2 H K \cdot 2\left(E G-F^{2}\right) H+K^{2}\left(E G-F^{2}\right)} \\
& =\frac{4 H\left(e g-f^{2}\right)-2\left(e g-f^{2}\right) H}{4 H^{2}\left(e g-f^{2}\right)-2 H\left(e g-f^{2}\right) \cdot 2 H+\frac{\left(e g-f^{2}\right)^{2}}{\left(E G-F^{2}\right)}} \\
& =\frac{2 H-H}{2 H^{2}-2 H^{2}+\frac{\left(e g-f^{2}\right)}{E G-F^{2}}}=\frac{H}{K}
\end{aligned}
$$

Portanto,

$$
\mathrm{H}(I I I, I I)=\frac{H}{K} .
$$

De forma análoga, temos 


$$
\begin{aligned}
\mathrm{K}(I I I, I I) & =\frac{e g-f^{2}}{(2 H e-K E)(2 H g-K G)-(2 H f-K F)^{2}} \\
& =\frac{e g-f^{2}}{4 H^{2}\left(e g-f^{2}\right)-4 H^{2} K\left(E G-F^{2}\right)+K^{2}\left(E G-F^{2}\right)} \\
& =\frac{1}{4 H^{2}-4 H^{2} K\left(\frac{E G-F^{2}}{e g-f^{2}}\right)+K^{2}\left(\frac{E G-F^{2}}{e g-f^{2}}\right)} \\
& =\frac{1}{4 H^{2}-4 H^{2} K \frac{1}{K}+K^{2} \frac{1}{K}} \\
& =\frac{1}{K} .
\end{aligned}
$$

Portanto,

$$
\mathrm{K}(I I I, I I)=\frac{1}{K}
$$

As expressões (1.11) e (1.12) ligadas ao par $(I I I, I I)$ serão úteis no futuro.

\subsubsection{Superfícies de Riemann}

Uma superficie de Riemann $M$ é uma variedade 2-dimensional, conexa de Hausdorff com estrutura diferenciável $\left\{\left(U_{\alpha}, Z_{\alpha}\right)\right\}$ tal que $\left\{Z_{\alpha}\left(U_{\alpha}\right)\right\}$ é uma cobertura aberta de $M$, onde

$$
Z_{\alpha}: U_{\alpha} \subset \mathbb{C} \rightarrow M
$$

é um homeomorfismo de um subconjunto aberto do plano complexo $\mathbb{C}$ de modo que as mudanças de parâmetros

$$
f_{\alpha \beta}=Z_{\alpha}^{-1} \circ Z_{\beta}: Z_{\beta}^{-1}\left(Z_{\beta}\left(U_{\beta}\right) \cap Z_{\alpha}\left(U_{\alpha}\right)\right) \rightarrow Z_{\alpha}^{-1}\left(Z_{\beta}\left(U_{\beta}\right) \cap Z_{\alpha}\left(U_{\alpha}\right)\right)
$$

são holomorfas sempre que $Z_{\alpha}\left(U_{\alpha}\right) \cap Z_{\beta}\left(U_{\beta}\right) \neq \emptyset$. Pelas equações de Cauchy-Riemann, concluímos que toda superfície de Riemann é orientável.

Se $u, v$ são parâmetros locais de uma parametrização $Z$, dizemos que os parâmetros são isotérmicos ou conformes se $\left\langle Z_{u}, Z_{u}\right\rangle=\left\langle Z_{v}, Z_{v}\right\rangle$ e $\left\langle Z_{u}, Z_{v}\right\rangle=0$.

Pode-se mostrar que toda superfície orientável $M$ possui uma estrutura de superfície de Riemann. O plano complexo $\mathbb{C}$, com a estrutura diferenciável $\{(\mathbb{C}, I d)\}$, e qualquer subconjunto aberto conexo de $\mathbb{C}$ são superfícies de Riemann; em particular, o disco unitário $D^{2}=\{z \in \mathbb{C} ;|z|<1\}$ é uma superfície de Riemann. A esfera de Riemann $\overline{\mathbb{C}}=\mathbb{C} \cup\{\infty\}$, com sua estrutura formada por duas parametrizações, a identidade definida em $\mathbb{C}$ e a aplicação $z \rightarrow \frac{1}{z}$ definida para $z \neq 0$ estendida a $\infty$ por $0 \mapsto \infty$, com a estrutura, é uma superfície de Riemann.

Uma aplicação contínua $f: M_{1} \rightarrow M_{2}$ entre duas superfícies de Riemann é dita holomorfa em $p \in M_{1}$ se, dada uma coordenada local $\left\{\left(V_{1}, Y_{1}\right)\right\}$ em $f(p)$, existe uma coordenada local $\left\{\left(U_{1}, X_{1}\right)\right\}$ em $p$ tal que $f\left(X_{1}\left(U_{1}\right)\right) \subset Y_{1}\left(V_{1}\right)$ e a aplicação $Y_{1}^{-1} \circ f \circ X_{1}: U_{1} \rightarrow \mathbb{C}$ é um holomorfismo em $X_{1}^{-1}(p)$. A função $f$ é dita holomorfa em um aberto de $M_{1}$ se for holomorfa em todos os pontos deste aberto. É claro que a definição dada é independente da escolha das coordenadas locais.

Se $f$ é injetiva e holomorfa, então $f^{-1}$ é holomorfa e $f$ é dita equivalência conforme. Neste caso, $M_{1}$ e $M_{2}$ são ditas conformemente equivalentes.

Lema 1.2. Seja $f: U \subset \mathbb{R}^{2} \rightarrow V \subset \mathbb{R}^{2}$ uma bijeção holomorfa entre abertos de $\mathbb{C}=\mathbb{R}^{2}$. Então, $f$ é uma 
aplicação conforme.

Demonstração. Escrevemos $f(x, y)=(u(x, y), v(x, y)),(x, y) \in U$. Como $f$ é holomorfa, vale as equações de Cauchy-Riemann

$$
\frac{\partial u}{\partial x}=\frac{\partial v}{\partial y}, \frac{\partial u}{\partial y}=-\frac{\partial v}{\partial x} .
$$

Sendo $\left\{e_{1}, e_{2}\right\}$ a base canônica de $\mathbb{R}^{2}$, segue que

$$
\begin{aligned}
\left\langle d f_{e_{1}}, d f_{e_{2}}\right\rangle & =\left\langle\frac{\partial f}{\partial x}, \frac{\partial f}{\partial y}\right\rangle=\left\langle\left(\frac{\partial u}{\partial x}, \frac{\partial v}{\partial x}\right),\left(\frac{\partial u}{\partial y}, \frac{\partial v}{\partial y}\right)\right\rangle \\
& =\frac{\partial u}{\partial x} \frac{\partial u}{\partial y}+\frac{\partial x}{\partial x} \frac{\partial v}{\partial y}=0 \\
\left\langle d f_{e_{1}}, d f_{e_{1}}\right\rangle & =\left\langle\frac{\partial f}{\partial x}, \frac{\partial f}{\partial x},\right\rangle=\left(\frac{\partial u}{\partial x}\right)^{2}+\left(\frac{\partial v}{\partial x}\right)^{2} \\
& =\left(\frac{\partial v}{\partial y}\right)^{2}+\left(\frac{\partial u}{\partial y}\right)^{2}=\left\langle d f_{e_{2}}, d f_{e_{2}}\right\rangle .
\end{aligned}
$$

Assim, $\left\langle d f_{e_{i}}, d f_{e_{j}}\right\rangle=\mu^{2} \delta_{i j}=\mu^{2}\left\langle e_{i}, e_{j}\right\rangle$, onde $\mu^{2}=\left(\frac{\partial u}{\partial x}\right)^{2}+\left(\frac{\partial v}{\partial x}\right)^{2}=\left(\frac{\partial v}{\partial y}\right)^{2}+\left(\frac{\partial u}{\partial y}\right)^{2}$. Agora, se $v$ é um campo de vetores qualquer em $U$, temos que

$$
\begin{aligned}
\left\langle d f_{v}, d f_{v}\right\rangle & =\left\langle d f\left(a_{1} e_{1}+a_{2} e_{2}\right), d f\left(a_{1} e_{1}+a_{2} e_{2}\right)\right\rangle \\
& =a_{1}^{2} \mu^{2}\left\langle e_{1}, e_{1}\right\rangle+a_{2} \mu^{2}\left\langle e_{2}, e_{2}\right\rangle \\
& =\mu^{2}\left(a_{1}^{2}+a_{2}^{2}\right)=\mu^{2}\langle v, v\rangle
\end{aligned}
$$

e portanto $f$ é uma aplicação conforme.

Seja agora $f: M_{1} \rightarrow M_{2}$ uma equivalência conforme entre duas superfícies de Riemann. Tomando $\{(U, X)\}$ e $\{(V, Y)\}$ como parametrizações isotérmicas em $M_{1}$ e $M_{2}$, respectivamente, tais que $f(X(U)) \subset$ $Y(V)$, então a aplicação

$$
\varphi=Y^{-1} \circ f \circ X: U \subset \mathbb{R}^{2} \rightarrow Y^{-1}(f(X(U))) \subset \mathbb{R}^{2}
$$

é uma bijeção holomorfa, e pelo lema anterior concluímos ser uma aplicação conforme. Como $X$ e $Y$ são isotérmicas, então são aplicações conformes. Assim, de $\varphi=Y^{-1} \circ f \circ X$ segue que $f=Y \circ \varphi \circ X^{-1}$ é um difeomorfismo, e por composição é localmente conforme. Portanto, $f$ é uma aplicação localmente conforme.

\subsubsection{Representação de Weierstrass}

Nesta seção apresentamos a Representação de Weierstrass para superfícies mínimas, tal representação fornece uma descrição explicita da superfície usando dois dados: uma função meromorfa e uma forma holomorfa. Para um estudo detalhado das formas diferenciáveis, citamos [9].

Seja $U \subset \mathbb{R}^{2}$ aberto simplesmente conexo e $\psi: U \rightarrow \mathbb{R}^{3}$ uma imersão, dizemos que $\psi$ é uma aplicação conforme se $\left|\psi_{u}\right|=\left|\psi_{v}\right|$ e $\left\langle\psi_{u}, \psi_{v}\right\rangle=0$, neste caso $\psi$ induz uma métrica em $U$ :

$$
d s^{2}=\lambda^{2}\left(d u^{2}+d v^{2}\right)
$$


onde $\lambda=\left|\psi_{u}\right|=\left|\psi_{v}\right|$. Dizemos que $(u, v)$ são parâmetros isotérmicos. A possibilidade de obter parâmetros isotérmicos é dada pelo seguinte teorema.

Teorema 1.5 (Existênca de parâmetros isotérmicos). Se $\psi: U \rightarrow \mathbb{R}^{3}$ é uma imersão, então existe um difeomorfismo $\varphi: U \rightarrow U$ (de classe $C^{k}$, sempre que $\psi \in C^{k}$ ) tal que $\tilde{\psi}=\psi \circ \varphi$ é uma aplicação conforme.

Demonstração. Ver [22].

Supondo $M$ uma superfície conexa e orientável e seja $x: M \rightarrow \mathbb{R}^{3}$ uma imersão de classe $C^{\infty}$, pelo teorema anterior cada ponto $p \in M$ possui uma vizinhança de parâmetros isotérmicos $(u, v)$. A métrica induzida sobre $M$ por $x$ será representada, localmente, em termos de tais parâmetros, por

$$
d s^{2}=\lambda^{2}|d z|^{2}
$$

onde $z=u+i v$. Assim, uma mudança de coordenadas de tais parâmetros é uma aplicação conforme.

Desde que $M$ é uma superfície orientável, podemos limitar-nos a famílias de parâmetros isotérmicos cuja mudança de coordenadas preserve a orientação do plano, tal superfície é uma superfície Riemanniana. Na superfície Riemanniana, definimos os operadores

$$
\begin{aligned}
\frac{\partial}{\partial z} & =\frac{1}{2}\left(\frac{\partial}{\partial u}-i \frac{\partial}{\partial v}\right), \\
\frac{\partial}{\partial \bar{z}} & =\frac{1}{2}\left(\frac{\partial}{\partial u}+i \frac{\partial}{\partial v}\right),
\end{aligned}
$$

e dizemos que $f$ é holomorfa se, e somente se, $\frac{\partial}{\partial \bar{z}} f=0$. Se $\frac{\partial}{\partial z} f=0$, dizemos que $f$ é anti-holomorfa.

Vamos agora considerar uma imersão $X:=\left(X_{1}, X_{2}, X_{3}\right): M \rightarrow \mathbb{R}^{3}$, na qual é possível definir parâmetros isotérmicos, então, já que

$$
\Delta X=2 H N
$$

temos

$$
\Delta=\frac{4}{\lambda^{2}} \frac{\partial}{\partial z} \frac{\partial}{\partial \bar{z}}=\frac{1}{\lambda^{2}}\left(\frac{\partial}{\partial u^{2}}+\frac{\partial}{\partial v^{2}}\right)
$$

consequentemente, temos a seguinte proposição.

Proposição 1.2. $X: M \rightarrow \mathbb{R}^{3}$ é uma imersão mínima se, e somente se, é harmônica.

Por outra parte, definindo $\phi=\frac{\partial}{\partial z} X$, temos

$$
\frac{\partial}{\partial \bar{z}} \phi=\frac{\partial}{\partial \bar{z}} \frac{\partial}{\partial z} X=\frac{\lambda^{2}}{4} \Delta X
$$

logo, $\phi$ é holomorfa se, e somente se, $X$ é harmônica.

Observação 1.4. Note que $\phi$ é uma função definida em $M$ localmente com valores na quádrica de $\mathbb{C}$ que satisfaz $z_{1}^{2}+z_{2}^{2}+z_{3}^{2}=0$, pois: Supondo $\phi=\left(\phi_{1}, \phi_{2}, \phi_{3}\right)=\frac{\partial}{\partial z} X$, então

$$
\phi_{k}=\frac{\partial}{\partial z} X_{k}=\frac{1}{2}\left(\frac{\partial}{\partial u} X_{k}-i \frac{\partial}{\partial v} X_{k}\right)
$$


dai,

$$
\begin{aligned}
\sum_{k} \phi_{k}^{2} & =\frac{1}{4}\left\{\sum_{k}\left(\frac{\partial}{\partial u} X_{k}\right)^{2}-\sum_{k}\left(\frac{\partial}{\partial v} X_{k}\right)^{2}-2 i \sum_{k} \frac{\partial}{\partial u} X_{k} \frac{\partial}{\partial v} X_{k}\right\} \\
& =\frac{1}{4}\left\{\left|X_{u}\right|^{2}-\left|X_{v}\right|^{2}-2 i\left\langle X_{u}, X_{v}\right\rangle\right\}=0
\end{aligned}
$$

$e$

$$
\begin{aligned}
|\phi|^{2}=\sum_{k}\left|\phi_{k}\right|^{2} & =\sum_{k}\left(\frac{1}{4}\left[\left(\frac{\partial}{\partial u} X_{k}\right)^{2}+\left(\frac{\partial}{\partial v} X_{k}\right)^{2}\right]\right) \\
& =\frac{1}{4}\left(\left|X_{u}\right|^{2}+\left|X_{v}\right|^{2}\right)=\frac{2}{4} \lambda=\frac{\lambda}{2}>0,
\end{aligned}
$$

assim, $\sum \phi_{k}^{2}=0$ e $|\phi|>0$.

Agora, suponha que $w=w(z)$ é uma mudança de parâmetros (ainda isotérmicos), então definindo uma função $\tilde{\phi}=\frac{\partial}{\partial w} X$, temos

$$
\phi=\frac{\partial}{\partial z} X=\frac{\partial}{\partial w} X \cdot \frac{\partial}{\partial z} w=\tilde{\phi} \frac{\partial}{\partial z}
$$

assim, se considerarmos as formas diferenciais $\alpha, \tilde{\alpha}: M \rightarrow \mathbb{C}^{3}$ dada por $\alpha=\phi d z$ e $\tilde{\alpha}=\tilde{\phi} d w$ em seus parâmetros $z, w$ respectivamente, teremos

$$
\alpha=\phi d z=\tilde{\phi} \frac{\partial}{\partial z} w d z=\tilde{\phi} d w=\tilde{\alpha}
$$

o que quer dizer que $\alpha$ está globalmente definida em $M$ com expressão local $\alpha=\left(\alpha_{1}, \alpha_{2}, \alpha_{3}\right)$ tal que $\alpha_{k}=\phi_{k} d z ; k=1,2,3$. Consequentemente, temos a seguinte proposição.

Proposição 1.3. Seja $X: M \rightarrow \mathbb{R}^{3}$ uma imersão. Então $\alpha=\phi d z$ é holomorfa sobre $M$ se, e somente se, $X$ é uma imersão mínima. Alias, $X=\operatorname{Re} \int^{z} \alpha$, onde a integral é tomada ao longo de qualquer caminho desde um ponto fixo ate $z \in M$.

Observação 1.5. Quando a parte real da integral de $\alpha$ ao longo de qualquer caminho fechado é zero, dizemos que a não tem períodos reais. A não existência de períodos reais para $\alpha$ é equivalente a que $\operatorname{Re}\left(\int^{z} \alpha\right)$ independa do caminho em $M$.

Teorema 1.6 (Represetação de Weierstrass). Sejam $\alpha_{1}, \alpha_{2}, \alpha_{3}$ formas diferenciáveis holomorfas sobre $M$ tais que

(a) $\sum_{k} \alpha_{k}^{2}=0,\left(\right.$ ou seja, $\left.\alpha_{k}=\phi_{k} d z \quad e \quad \sum_{k} \phi_{k}^{2}=0\right)$,

(b) $\sum_{k}\left|\alpha_{k}\right|^{2}>0, \quad e$

(c) Cada $\alpha_{k}$ não tem periodos reais em $M$.

Então, a aplicação $X: M \rightarrow \mathbb{R}^{3}$ definida por $X=\left(X_{1}, X_{2}, X_{3}\right)$ com $X_{k}=\operatorname{Re} \int^{z} \alpha_{k}$ é uma imersão minima.

Observação 1.6. A condição (c) é necessária para garantir que $\operatorname{Re} \int_{p_{0}}^{z} \alpha_{k}$ depende só do ponto final $z$. Assim, cada $X_{k}$ é bem definida independentemente do caminho desde $p_{0}$ ate $z$. (b) garante que $X$ seja imersão. 
É possível dar uma descrição das soluções a $\alpha_{1}^{2}+\alpha_{2}^{2}+\alpha_{3}^{2}=0$ sobre $M$. Para isso, suponha que $\alpha_{1} \neq i \alpha_{2}$ ( $M$ não imersa num plano), definimos a função meromorfa $g$ e a forma holomorfa $w$ por:

$$
\begin{aligned}
& g=\frac{\alpha_{3}}{\alpha_{2}-i \alpha_{2}}, \\
& w=\alpha_{1}-i \alpha_{2},
\end{aligned}
$$

localmente $\alpha_{k}=\phi_{k} d z$, então

$$
w=\alpha_{1}-i \alpha_{2}=\left(\phi_{1}-i \phi_{2}\right) d z
$$

definindo $f:=\phi_{1}-i \phi_{2}$ temos que $f$ é uma função holomorfa e $w=f d z$. Por outra parte, nos termos de $g$ e $w$ temos:

$$
\begin{aligned}
& \alpha_{1}=\frac{w}{2}\left(1-g^{2}\right), \\
& \alpha_{2}=i \frac{w}{2}\left(1+g^{2}\right), \\
& \alpha_{3}=g w .
\end{aligned}
$$

Assim, a imersão mínima é dada por

$$
\left\{\begin{array}{l}
X_{1}=\operatorname{Re} \int^{z} \alpha_{1}=\operatorname{Re} \int^{z} \frac{w}{2}\left(1-g^{2}\right) d z \\
X_{2}=\operatorname{Re} \int^{z} \alpha_{2}=\operatorname{Re} \int^{z} i \frac{w}{2}\left(1+g^{2}\right) d z \\
X_{3}=\operatorname{Re} \int^{z} \alpha_{3}=\operatorname{Re} \int^{z} g w d z
\end{array}\right.
$$

A expressão em (1.14) é chamada Fórmula de Representação de Weierstrass para superfícies mínimas em $\mathbb{R}^{3}$.

Observação 1.7. Note que $N=\frac{1}{1+|g|^{2}}\left(2 \operatorname{Re} g, 2 \operatorname{Im} g,|g|^{2}-1\right)$. Se $\pi$ é a projeção estereográfica estendida, $\pi \circ N=g$, assim $g$ é a aplicação normal da imersão.

Exemplo 1.5. Seja $M=\mathbb{C}, g(z)=-i e^{z}, w=e^{-z} d z$. Observe que $g$ não tem polos $e w$ não tem zeros em $\mathbb{C}$. Então

$$
\begin{aligned}
\alpha_{1} & =\frac{e^{-z}}{2}\left(1+e^{2 z}\right) d z \\
& =\frac{e^{-z}+e^{z}}{2} d z \\
& =\cosh z d z \\
\alpha_{2} & =\frac{i e^{-z}}{2}\left(1-e^{2 z}\right) d z \\
& =i\left(\frac{e^{-z}-e^{z}}{2}\right) d z \\
& =-i \sinh z d z \\
\alpha_{3} & =-i e^{z} e^{-z} d z \\
& =-i d z .
\end{aligned}
$$


Logo,

$$
\begin{aligned}
X_{1} & =\operatorname{Re} \int_{0}^{z} \cosh z d z=\operatorname{Re}(\sinh z)=\operatorname{Re}(-i \sin i z) \\
& =\operatorname{Re}[-i \sin (i u-v)]=\operatorname{Re}[-i(\sin i u \cos v-\cos i u \sin v)] \\
& =\operatorname{Re}[-i(i \sinh u \cos v+\sin v \cosh u)] \\
& =\operatorname{Re}(\sinh u \cos v+i \sin v \cosh u) \\
& =\sinh u \cos v \\
X_{2} & =\operatorname{Re} \int_{0}^{z}-i \sinh z d z=\operatorname{Re}(-i \cosh z)=\operatorname{Re}(-i \cos i z) \\
& =\operatorname{Re}[-i \cos (i u-v)]=\operatorname{Re}[-i(\cos i u \cos v+\sin i u \sin v)] \\
& =\operatorname{Re}[-i(\cosh u \cos v+i \sinh u \sin v)] \\
& =\operatorname{Re}(-i \cos u \cos v+\sinh u \sin v) \\
& =\sinh u \sin v \\
X_{3} & =\operatorname{Re} \int_{0}^{z}-i d z=\operatorname{Re}(i z) \\
& =-\operatorname{Re}(i u-v)=v
\end{aligned}
$$

Como $\cosh z, \sinh z$, e o produto são holomorfos, temos para todo caminho fechado $\gamma$ que $\int_{\gamma} \alpha_{k}=0$, isto é, $\alpha_{k}$ não tem períodos reais. Logo da equação (1.14) temos

$$
\left\{\begin{array}{l}
X_{1}=\sinh u \cos v \\
X_{2}=\sinh u \sin v \\
X_{3}=v
\end{array}\right.
$$

chamando $t=\sinh u$, temos que $X=(t \cos v, t \sin v, v)$ é o Helicóide.

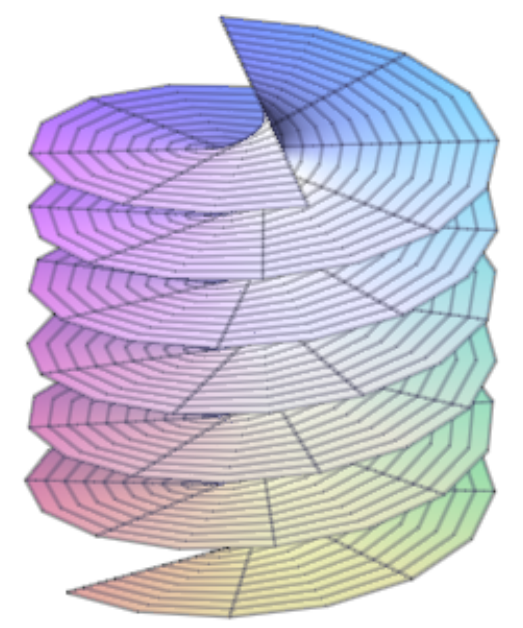




\section{Capítulo}

2

\section{Geometria de Laguerre}

A geometria de Laguerre é essencialmente a geometria Euclidiana de esferas e planos orientados, seu estudo iniciou com J. Weingarten em 1888. Posteriormente foi desenvolvido por Blaschke desde o ano 1924 (ver [6], [3], [4], [5]) e no último século retomou grande interesse ([1], [13], [15], [16], [20], [12], [21]). Um dos objetivos desta geometria é estudar as superfícies críticas do volume da métrica de Laguerre, chamadas superfícies mínimas de Laguerre. Nesta seção apresentamos os conceitos de orientação e contato de esferas e planos em termos de suas coordenadas esféricas. Introduzimos o grupo de transformações de Laguerre e mostramos a invariância da métrica de Laguerre pela ação deste grupo. Finalizamos com um teorema de caracterização das superfícies mínimas de Laguerre em termos de sua curvatura média e Gaussiana.

\subsection{Geometria das esferas orientadas em $\mathbb{R}^{3}$}

Nesta seção apresentamos a geometria de esferas orientadas em $\mathbb{R}^{3}$. Para maiores detalhes sobre esferas orientadas na geometria de Lie referimos aos livros [8] e [7].

Seja $U \mathbb{R}^{3}$ o fibrado tangente unitário de $\mathbb{R}^{3}$, o qual pode ser mergulhado em $\mathbb{R}^{6}$ por

$$
U \mathbb{R}^{3}=\left\{(x, \xi) \mid x \in \mathbb{R}^{3}, \xi \in S^{2}\right\} \subset \mathbb{R}^{3} \times S^{2} \subset \mathbb{R}^{6},
$$

existem duas classes de esferas orientadas em $U \mathbb{R}^{3}$, a primeira delas corresponde propriamente a uma esfera e a segunda a um plano visto como um caso de esfera degenerada de raio infinitamente grande, ambos no espaço $\mathbb{R}^{3}$.

Definição 2.1. Uma esfera orientada em $U \mathbb{R}^{3}$ centrada em $p$ com raio $r$ é uma subvariedade 2dimensional em $U \mathbb{R}^{3}$ dada por

$$
S(p, r)=\left\{(x, \xi) \in U \mathbb{R}^{3} \mid x-p=r \xi\right\}, p \in \mathbb{R}^{3}, r \in \mathbb{R} .
$$

Geometricamente $S(p, r)$ é uma esfera orientada com raio $r$ em $\mathbb{R}^{3}$. Se $r>0$ (respectivamente $r<0$ ), então a esfera é orientada para fora (respectivamente para dentro). Se $r=0$, então $S(p, 0)$ consiste de 
todos os vetores unitários em $p$, nesse caso chamamos a $S(p, 0)$ ponto esférico.

Definição 2.2. Um plano orientado em $U \mathbb{R}^{3}$ com vetor constante $v \in S^{2}$ e constante a $\in \mathbb{R}$ é uma subvariedade 2-dimensional em $U \mathbb{R}^{3}$ dada por

$$
P(v, a)=\left\{(x, v) \in U \mathbb{R}^{3} \mid x \cdot v=a\right\}
$$

Geometricamente, $P(v, a)$ é o plano orientado $\left\{x \in \mathbb{R}^{3} \mid x \cdot v=a\right\}$ em $\mathbb{R}^{3}$ com vetor normal $v$. Denotamos por $\Sigma$ o conjunto constituído por todas as esferas orientadas e planos orientados em $U \mathbb{R}^{3}$.

Definição 2.3. Sejam $\Gamma_{1}, \Gamma_{2} \in \Sigma$. Dizemos que $\Gamma_{1}$ e $\Gamma_{2}$ têm contato orientado se $\Gamma_{1}=\Gamma_{2}$ ou se a intersecção $\Gamma_{1} \cap \Gamma_{2}$ é um único ponto $(x, \xi) \in U \mathbb{R}^{3}$.

Geometricamente, $\Gamma_{1}$ e $\Gamma_{2}$ têm contato orientado em $(x, \xi)$ se, e somente se, elas são esferas orientadas (ou planos orientados) em $\mathbb{R}^{3}$ tangentes em $x$ com o mesmo campo normal unitário $\xi$. Para determinar uma forma analítica da condição de existência de contato orientado, introduzimos as seguintes definições.

Definição 2.4. Definimos o espaço pseudo-Euclidiano $\mathbb{R}_{2}^{6}$ como $\mathbb{R}^{6}$ munido do produto escalar $\langle$,$\rangle ,$ dado por

$$
\langle x, y\rangle=-x_{1} y_{1}+x_{2} y_{2}+x_{3} y_{3}+x_{4} y_{4}+x_{5} y_{5}-x_{6} y_{6}
$$

para $x=\left(x_{1}, \cdots, x_{6}\right), y=\left(y_{1}, \cdots, y_{6}\right) \in \mathbb{R}^{6}$.

Definição 2.5. Definimos o cone de luz $\mathrm{C}^{5}$ em $\mathbb{R}_{2}^{6}$ por

$$
\mathrm{C}^{5}=\left\{x \in \mathbb{R}_{2}^{6} \mid\langle x, x\rangle=0, x \neq 0\right\} \subset \mathbb{R}_{2}^{6}
$$

Definição 2.6. No espaço projetivo real $\mathbb{R} P^{5}$, definimos a quádrica $\mathrm{Q}^{4}$ por

$$
\mathrm{Q}^{4}=\left\{[x] \in \mathbb{R} P^{5} \mid\langle x, x\rangle=0, x \neq 0\right\} .
$$

Então, pode-se associar uma esfera orientada $S(p, r) \in \Sigma$ a um vetor $\gamma \in \mathrm{C}^{5}$ dado por

$$
\gamma=\left(\frac{1}{2}\left(1+|p|^{2}-r^{2}\right), \frac{1}{2}\left(1-|p|^{2}+r^{2}\right), p,-r\right)
$$

e associar um plano orientado $P(v, a) \in \Sigma$ a um vetor $\gamma^{*} \in \mathrm{C}^{5}$ dado por

$$
\gamma^{*}=(a,-a, v, 1)
$$

onde $|\cdot|$ denota o produto Euclidiano em $\mathbb{R}^{3}$. Chamamos a $\gamma, \gamma^{*}$ de coordenada esférica de $S(p, r)$ e $P(v, a)$, respectivamente.

Para qualquer $\Gamma \in \Sigma$, denotamos por $\gamma$ sua coordenada dada em (2.1) e (2.2). Então, temos que a correspondência $\Gamma \in \Sigma \leftrightarrow \gamma \in \mathrm{C}^{5}$ define uma bijeção de $\Sigma$ em $\mathrm{C}^{5} \backslash\{\wp\}$, onde

$$
\wp=(1,-1, \mathbf{0}, 0), \mathbf{0} \in \mathbb{R}^{3} .
$$


Geometricamente, o ponto

$$
\wp=\lim _{|p| \rightarrow \infty} \frac{1}{|p|^{2}}\left(\frac{1}{2}\left(1+|p|^{2}\right), \frac{1}{2}\left(1-|p|^{2}\right), p, 0\right)
$$

em $\mathrm{C}^{5}$ é a coordenada do ponto esférico no $\infty$ de $\mathbb{R}^{3}$.

A seguir, vamos fazer uma análise dos contatos orientados entre elementos de $\Sigma$. O objetivo deste análise é estabelecer uma relação entre o contato orientado e as coordenadas esféricas em $\mathrm{C}^{5}$.

- Caso I: Duas esferas orientadas diferentes.

Sejam $S\left(p_{1}, r_{1}\right)$ e $S\left(p_{2}, r_{2}\right)$ duas esferas orientadas cujas coordenadas esféricas são

$$
\begin{aligned}
& \gamma_{1}=\left(\frac{1}{2}\left(1+\left|p_{1}\right|^{2}-r_{1}^{2}\right), \frac{1}{2}\left(1-\left|p_{1}\right|^{2}+r_{1}^{2}\right), p_{1},-r_{1}\right), \\
& \gamma_{2}=\left(\frac{1}{2}\left(1+\left|p_{2}\right|^{2}-r_{2}^{2}\right), \frac{1}{2}\left(1-\left|p_{2}\right|^{2}+r_{2}^{2}\right), p_{2},-r_{2}\right),
\end{aligned}
$$

respectivamente. Em princípio, podem ocorrer os seguintes casos
i. $p_{1}=p_{2}, r_{1} \neq r_{2}$,
ii. $p_{1} \neq p_{2}, r_{1}=r_{2}$,
iii. $p_{1} \neq p_{2}, r_{1} \neq r_{2}$.

As duas esferas possuem contato orientado se existe um único ponto $(x, \xi) \in S\left(p_{1}, r_{1}\right) \cap S\left(p_{2}, r_{2}\right)$, consequentemente, deve-se satisfazer que

$$
x-p_{1}=r_{1} \xi
$$

e

$$
x-p_{2}=r_{2} \xi,
$$

daí, temos que

$$
p_{1}-p_{2}=\left(r_{2}-r_{1}\right) \xi
$$

e já que $\xi \in S^{2}$, obtemos que $\left|p_{1}-p_{2}\right|=\left|r_{2}-r_{1}\right|$. Portanto, o único caso possível é o caso (iii).

Agora, observe que se existir $(x, \xi) \neq(\bar{x}, \bar{\xi}) \in S\left(p_{1}, r_{1}\right) \cap S\left(p_{2}, r_{2}\right)$ teríamos que

$$
\bar{x}-p_{1}=r_{1} \bar{\xi}
$$

e

$$
\bar{x}-p_{2}=r_{2} \bar{\xi}
$$

logo, de (2.4) e (2.6) temos que

$$
x-r_{1} \xi=\bar{x}-r_{1} \bar{\xi}
$$

e logo,

$$
x-\bar{x}=r_{1}(\xi-\bar{\xi}) .
$$


Por outra parte, de (2.5) e (2.7) temos que

$$
x-\bar{x}=r_{2}(\xi-\bar{\xi})
$$

igualando (2.8) e (2.9), temos

$$
r_{1}(\xi-\bar{\xi})=r_{2}(\xi-\bar{\xi})
$$

assim,

$$
\left(r_{1}-r_{2}\right)(\xi-\bar{\xi})=0
$$

e como $r_{1} \neq r_{2}$, obtemos que $\xi=\bar{\xi}$, o que é uma contradição. Portanto, o ponto de intersecção de $S\left(p_{1}, r_{1}\right)$ e $S\left(p_{2}, r_{2}\right)$ é único.

Analisamos agora o produto escalar das coordenadas esféricas $\gamma_{1}$ e $\gamma_{2}$, temos

$$
\begin{aligned}
\left\langle\gamma_{1}, \gamma_{2}\right\rangle= & \left\langle\left(\frac{1}{2}\left(1+\left|p_{1}\right|^{2}-r_{1}^{2}\right), \frac{1}{2}\left(1-\left|p_{1}\right|^{2}+r_{1}^{2}\right), p_{1},-r_{1}\right),\left(\frac{1}{2}\left(1+\left|p_{2}\right|^{2}-r_{2}^{2}\right), \frac{1}{2}\left(1-\left|p_{2}\right|^{2}+r_{2}^{2}\right), p_{2},-r_{2}\right)\right\rangle \\
= & -\frac{1}{4}\left[\left(1+\left|p_{1}\right|^{2}-r_{1}^{2}\right)\left(1+\left|p_{2}\right|^{2}-r_{2}^{2}\right)\right]+\frac{1}{4}\left[\left(1-\left|p_{1}\right|^{2}+r_{1}^{2}\right)\left(1-\left|p_{2}\right|^{2}+r_{2}^{2}\right)\right]+p_{1} \cdot p_{2}-r_{1} r_{2} \\
= & -\frac{1}{4}\left[1+\left(\left|p_{2}\right|^{2}-r_{2}^{2}\right)+\left(\left|p_{1}\right|^{2}-r_{1}^{2}\right)+\left(\left|p_{1}\right|^{2}-r_{1}^{2}\right)\left(\left|p_{2}\right|^{2}-r_{2}^{2}\right)\right] \\
& +\frac{1}{4}\left[1+\left(-\left|p_{2}\right|^{2}+r_{2}^{2}\right)+\left(-\left|p_{1}\right|^{2}+r_{1}^{2}\right)+\left(-\left|p_{1}\right|^{2}+r_{1}^{2}\right)\left(-\left|p_{2}\right|^{2}+r_{2}^{2}\right)\right]+p_{1} \cdot p_{2}-r_{1} r_{2} \\
= & -\frac{1}{2}\left(\left|p_{2}\right|^{2}-r_{2}^{2}\right)-\frac{1}{2}\left(\left|p_{1}\right|^{2}-r_{1}^{2}\right)+p_{1} \cdot p_{2}-r_{1} r_{2} \\
= & -\frac{1}{2}\left(\left|p_{1}\right|^{2}-2 p_{1} \cdot p_{2}+\left|p_{2}\right|^{2}\right)+\frac{1}{2}\left(r_{2}^{2}-2 r_{1} r_{2}+r_{1}^{2}\right) \\
= & -\frac{1}{2}\left|p_{1}-p_{2}\right|^{2}+\frac{1}{2}\left(r_{2}-r_{1}\right)^{2} .
\end{aligned}
$$

Logo, se $\left\langle\gamma_{1}, \gamma_{2}\right\rangle=0$, teríamos que $\left|p_{1}-p_{2}\right|^{2}=\left(r_{2}-r_{1}\right)^{2}$, de onde $\left|p_{1}-p_{2}\right|=\left|r_{2}-r_{1}\right|$, portanto $S\left(p_{1}, r_{1}\right)$ e $S\left(p_{2}, r_{2}\right)$ são de contato orientado. Reciprocamente, se $S\left(p_{1}, r_{1}\right)$ e $S\left(p_{1}, r_{2}\right)$ são de contato orientado, temos que $\left|p_{1}-p_{2}\right|=\left|r_{2}-r_{1}\right|$, de onde $\left\langle\gamma_{1}, \gamma_{2}\right\rangle=0$. Temos assim que as esferas orientadas são de contato orientado se e somente se $\left\langle\gamma_{1}, \gamma_{2}\right\rangle=0$. Geometricamente, temos em $U \mathbb{R}^{3}$ a seguinte figura.
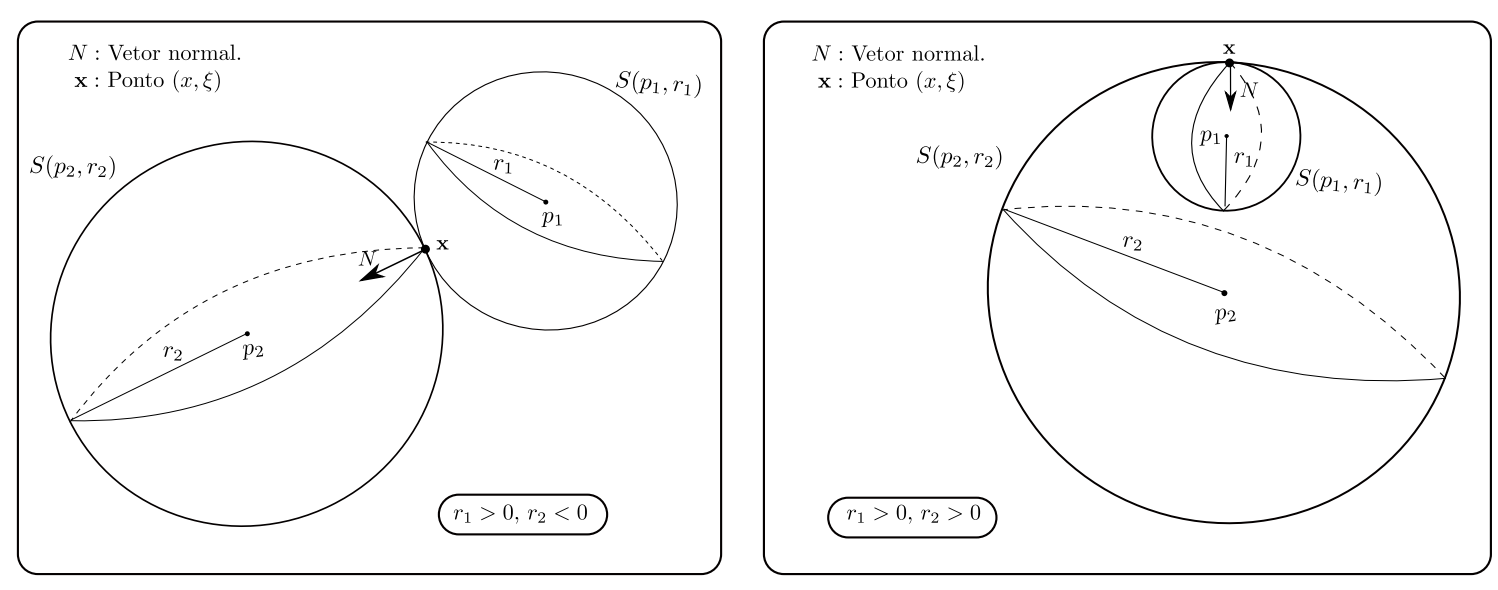
Observação 2.1. Se tomamos $r_{1}=r_{2}=0$, este caso inclui o caso de contato orientado entre dois pontos esféricos $S\left(p_{1}, 0\right)$ e $S\left(p_{2}, 0\right)$, onde de uma maneira rápida, obtemos que devem ser o mesmo e trivialmente é satisfeita a condição da existência de contato orientado se, e somente se, o produto das suas coordenadas esféricas é nulo. De fato, se em (2.10) supomos que $\left\langle\gamma_{1}, \gamma_{2}\right\rangle=0$ obtemos que $p_{1}=p_{2}$, e se a priori supomos que elas possuem contato orientado obtemos que $p_{1}=p_{2}$ e dai segue que $\left\langle\gamma_{1}, \gamma_{2}\right\rangle=0$.

- Caso II: Ponto esférico e uma esfera orientada.

Sejam $S(p, 0)$ e $S(q, r)$ um ponto esférico e uma esfera orientada, respectivamente. Suas coordenadas esféricas são

$$
\begin{aligned}
\gamma_{1} & =\left(\frac{1}{2}\left(1+|p|^{2}\right), \frac{1}{2}\left(1-|p|^{2}\right), p, 0\right), \\
\gamma_{2} & =\left(\frac{1}{2}\left(1+|q|^{2}-r^{2}\right), \frac{1}{2}\left(1-|q|^{2}+r^{2}\right), q,-r\right),
\end{aligned}
$$

respectivamente. Supondo que tem contato orientado, deve existir um único $(x, \xi) \in S(p, 0) \cap S(q, r)$, assim, deve-se satisfazer

$$
x-p=0,
$$

e

$$
x-q=r \xi
$$

note que supondo a existência de outro elemento $(\bar{x}, \bar{\xi}) \in S(p, 0) \cap S(q, r)$, procedendo analogamente ao caso anterior obteremos que de fato $(\bar{x}, \bar{\xi})=(x, \xi)$, o que garante a unicidade do contato. Por outra parte, usando a equação (2.11) na equação $(2.12)$ e do fato $\xi \in S^{2}$, temos que

$$
|p-q|=|r|
$$

Analisamos agora o produto escalar das coordenadas esféricas $\gamma_{1}$ e $\gamma_{2}$, temos

$$
\begin{aligned}
\left\langle\gamma_{1}, \gamma_{2}\right\rangle & =\left\langle\left(\frac{1}{2}\left(1+|p|^{2}\right), \frac{1}{2}\left(1-|p|^{2}\right), p, 0\right),\left(\frac{1}{2}\left(1+|q|^{2}-r^{2}\right), \frac{1}{2}\left(1-|q|^{2}+r^{2}\right), q,-r\right)\right\rangle \\
& =-\frac{1}{4}\left[\left(1+|p|^{2}\right)\left(1+|q|^{2}-r^{2}\right)\right]+\frac{1}{4}\left[\left(1-|p|^{2}\right)\left(1-|q|^{2}+r^{2}\right)\right]+p \cdot q \\
& =-\frac{|p|^{2}}{4}-\frac{1}{4}\left(|q|^{2}-r^{2}\right)-\frac{|p|^{2}}{4}\left(|q|^{2}-r^{2}\right)-\frac{|p|^{2}}{4}+\frac{1}{4}\left(-|q|^{2}+r^{2}\right)-\frac{|p|^{2}}{4}\left(-|q|^{2}+r^{2}\right)+p \cdot q \\
& =-\frac{|p|^{2}}{2}-\frac{1}{2}\left(|q|^{2}-r^{2}\right)+p \cdot q \\
& =-\frac{1}{2}\left(|p|^{2}-2 p \cdot q+|q|^{2}\right)+\frac{r^{2}}{2} \\
& =-\frac{1}{2}\left(|p-q|^{2}\right)+\frac{r^{2}}{2} .
\end{aligned}
$$

Agora, supondo que $\left\langle\gamma_{1}, \gamma_{2}\right\rangle=0$, usando a equação (2.13) teríamos que $S(p, 0)$ e $S(q, r)$ possuem contato orientado. Reciprocamente, se na equação (2.14) supomos que a equação (2.13) é satisfeita, teríamos que $\left\langle\gamma_{1}, \gamma_{2}\right\rangle=0$. Temos então que o ponto esférico e a esfera orientada são de contato orientado se, e somente se, $\left\langle\gamma_{1}, \gamma_{2}\right\rangle=0$. Geometricamente, temos em $U \mathbb{R}^{3}$ a seguinte figura. 


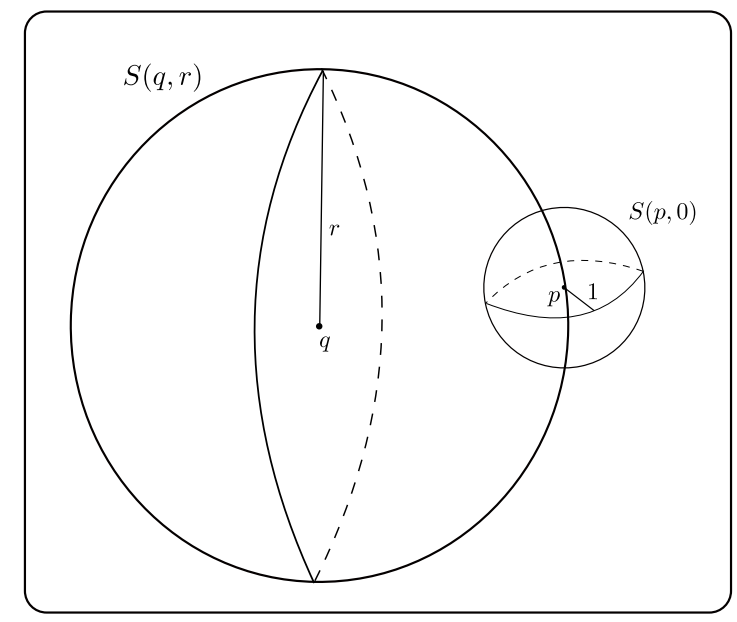

- Caso III: Esfera orientada e um plano.

Sejam $S(p, r)$ e $P(v, a)$ uma esfera orientada e um plano orientado, respectivamente. Suas coordenadas esféricas são

$$
\begin{aligned}
\gamma_{1} & =\left(\frac{1}{2}\left(1+|p|^{2}-r^{2}\right), \frac{1}{2}\left(1-|p|^{2}+r^{2}\right), p,-r\right) \\
\gamma_{2} & =(a,-a, v, 1),
\end{aligned}
$$

respectivamente. Já que neste caso o vetor normal de $P(v, a)$ não pode mudar, elas possuem contato orientado se existe um único $(x, v) \in S(p, r) \cap P(v, a)$, assim, deve-se satisfazer

$$
x-p=r v,
$$

e

$$
x \cdot v=a,
$$

$\operatorname{logo}$, como $v \in S^{2}$, obtemos da equação (2.15) que

$$
(x-p) \cdot v=r,
$$

e usando a equação (2.16)

$$
x \cdot v-p \cdot v=r=a-p \cdot v,
$$

assim,

$$
p \cdot v=-r+a .
$$

Agora, observe que se existir $(x, v) \neq(\bar{x}, v) \in S(p, r) \cap P(v, a)$, teríamos que

$$
\bar{x}-p=r v,
$$

e

$$
\bar{x} \cdot v=a,
$$

mas, das equações equações (2.15) e (2.18) temos que

$$
x-r v=p=\bar{x}-r v,
$$


ou equivalentemente

$$
x-\bar{x}=0,
$$

contradizendo a hipótese de ser $x \neq \bar{x}$, portanto, a unicidade do contato está garantida.

Analisamos agora o produto escalar

$$
\begin{aligned}
\left\langle\gamma_{1}, \gamma_{2}\right\rangle & =\left\langle\left(\frac{1}{2}\left(1+|p|^{2}-r^{2}\right), \frac{1}{2}\left(1-|p|^{2}+r^{2}\right), p,-r\right),(a,-a, \xi, 1)\right\rangle \\
& =-\frac{a}{2}\left(1+|p|^{2}-r^{2}\right)-\frac{a}{2}\left(1-|p|^{2}+r^{2}\right)+p \cdot \xi+r \\
& =-a+p \cdot \xi+r .
\end{aligned}
$$

Logo, se $\left\langle\gamma_{1}, \gamma_{2}\right\rangle=0$, teríamos da equação (2.17) que $r-a=-p \cdot v$, ou seja $S(p, r)$ e $P(v, a)$ tem contato orientado. Reciprocamente, se $S(p, r)$ e $P(v, a)$ tem contato orientado, temos $r-a=-p \cdot v$, de onde $\left\langle\gamma_{1}, \gamma_{2}\right\rangle=0$. Logo, a esfera orientada e o plano orientado tem contato orientado se, e somente se $\left\langle\gamma_{1}, \gamma_{2}\right\rangle=0$. Então, uma esfera orientada e um plano orientado tem contato orientado se, e somente se $\left\langle\gamma_{1}, \gamma_{2}\right\rangle=0$. Geometricamente, temos em $U \mathbb{R}^{3}$ a seguinte figura.

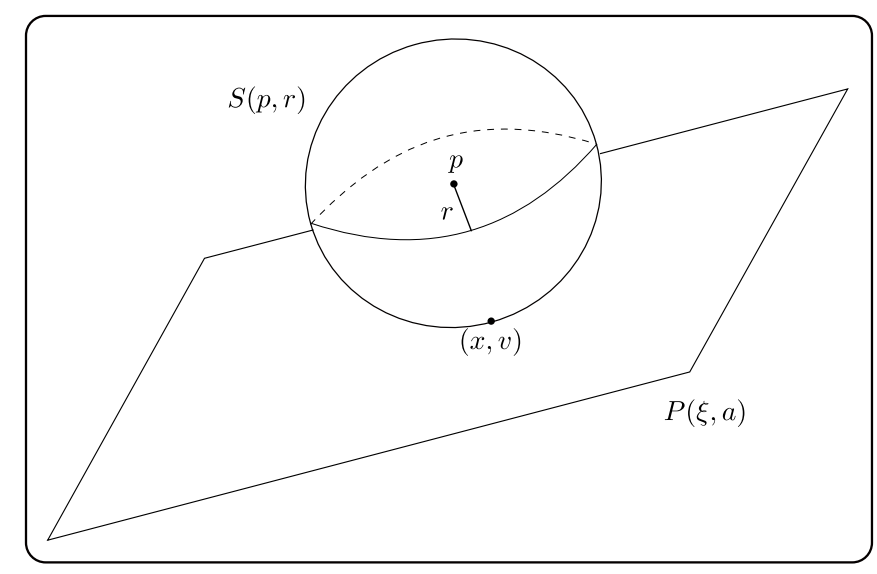

Observação 2.2. Este caso inclui o caso de contato orientado entre um ponto esférico $S(p, 0)$ e um plano orientado $P(v, a)$. Geometricamente, possuem contato orientado se, e somente se, o centro $p$ do ponto esférico está no plano $P(v, a)$.

- Caso IV: Dois planos orientados.

Sejam $P\left(v_{1}, a_{1}\right)$ e $P\left(v_{2}, a_{2}\right)$ dois planos orientados cujas coordenadas esféricas são, respectivamente

$$
\gamma_{1}=\left(a_{1},-a_{1}, v_{1}, 1\right)
$$

e

$$
\gamma_{2}=\left(a_{2},-a_{2}, v_{2}, 1\right)
$$

Então, possuem contato orientado se existe um único ponto $(x, v) \in P\left(v_{1}, a_{1}\right) \cap P\left(v_{2}, a_{2}\right)$. Portanto, 
deve-se satisfazer

$$
\begin{gathered}
v=v_{1}=v_{2}, \\
x \cdot v=a_{1}, \\
x \cdot v=a_{2},
\end{gathered}
$$

então, definimos $a:=a_{1}=a_{2}$, assim teríamos que dois planos orientados tem contato orientado só no caso trivial de serem o mesmo. Note também, já que sendo que $v \in S^{2}$, temos

$$
\begin{aligned}
\left\langle\gamma_{1}, \gamma_{2}\right\rangle & =\langle(a,-a, v, 1),(a,-a, v, 1)\rangle \\
& =-a^{2}+a^{2}+|v|^{2}-1 \\
& =0
\end{aligned}
$$

assim, ter contato orientado implica $\left\langle\gamma_{1}, \gamma_{2}\right\rangle=0$. Aliás, supondo a priori que $\left\langle\gamma_{1}, \gamma_{2}\right\rangle=0$ implicaria que $v_{1} \cdot v_{2}=1$ o que significaria que $\measuredangle\left(v_{1}, v_{2}\right)=0$, concluindo que os planos $P\left(v_{1}, a_{1}\right)$ e $P\left(v_{2}, a_{2}\right)$ são os mesmos, ou seja, tem contato orientado. Portanto, dois planos orientados tem contato orientado se, e somente se $\left\langle\gamma_{1}, \gamma_{2}\right\rangle=0$.

Concluímos então a seguinte proposição.

Proposição 2.1. Duas esferas orientadas $\Gamma_{1}, \Gamma_{2} \in \Sigma$ têm contato orientado se, e somente se, suas coordenadas esféricas $\gamma_{1}, \gamma_{2} \in \mathrm{C}^{5}$, respectivamente, satisfazem

$$
\left\langle\gamma_{1}, \gamma_{2}\right\rangle=0
$$

Observação 2.3. De (2.1), (2.2) e da definição de §, temos que se $\gamma$ é coordenada de alguma esfera $S(p, r)$, então

$$
\begin{aligned}
\langle\gamma, \wp\rangle & =\left\langle\left(\frac{1}{2}\left(1+|p|^{2}-r^{2}\right), \frac{1}{2}\left(1-|p|^{2}+r^{2}\right), p,-r\right),(1,-1, \mathbf{0}, 0)\right\rangle \\
& =-\frac{1}{2}\left(1+|p|^{2}-r^{2}\right)-\frac{1}{2}\left(1-|p|^{2}+r^{2}\right) \\
& =-1
\end{aligned}
$$

por outra parte, se $\gamma^{*}$ é coordenada de algum plano $P(v, a)$, então

$$
\begin{aligned}
\left\langle\gamma^{*}, \wp\right\rangle & =\langle(a,-a, v, 1),(1,-1, \mathbf{0}, 0)\rangle \\
& =-a+a \\
& =0 .
\end{aligned}
$$

Assim, sendo que $\Sigma=\{$ esferas orientadas $\} \cup\{$ planos orientados $\}$ temos a seguinte propriedade.

Propriedade 2.1. Seja $\Gamma \in \Sigma$ uma esfera orientada com coordenada $\gamma$, então

- $\Gamma$ é uma esfera orientada se, e somente se, $\langle\gamma, \wp\rangle \neq 0$.

- $\Gamma$ é um plano orientado se, e somente se, $\langle\gamma, \wp\rangle=0$.

Notemos que para qualquer ponto $\left(x_{0}, \xi_{0}\right) \in U \mathbb{R}^{3}$ é determinado um único conjunto de esferas orientadas de contato orientado em $x_{0} \in \mathbb{R}^{3}$ com o mesmo vetor normal unitário $\xi_{0}$. Geometricamente temos 


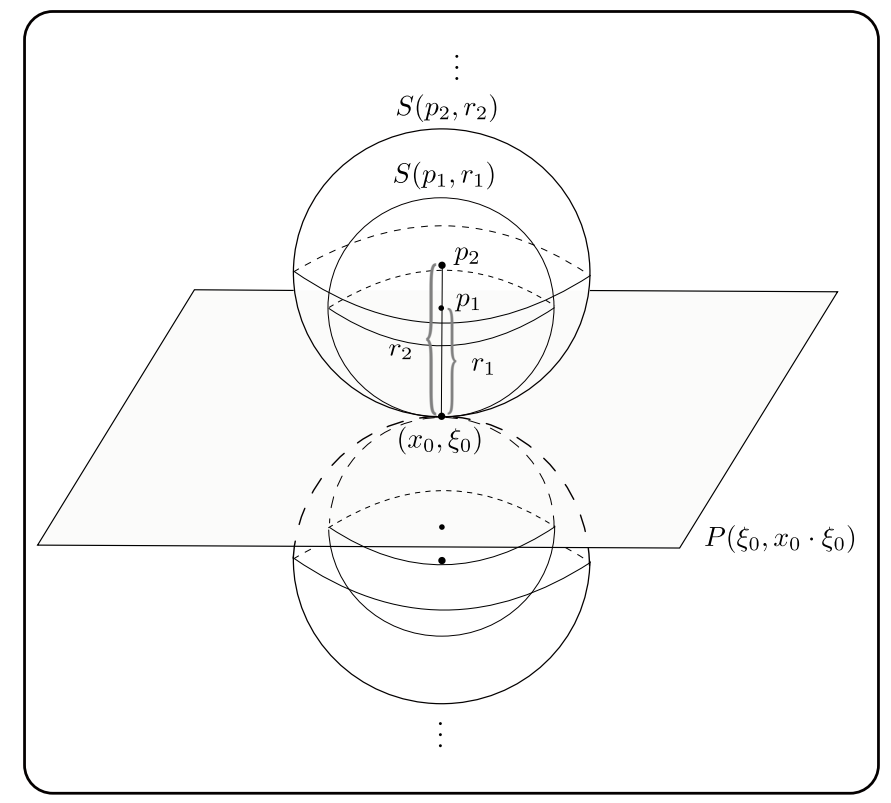

Onde as esferas na parte superior correspondem a raios positivos e as esferas da parte inferior a raios negativos. Notemos também que é determinado um único plano $P\left(\xi_{0}, x_{0} \cdot \xi_{0}\right)$ de coordenada esférica $\gamma_{1}$ neste feixe além de um único ponto esférico $S\left(x_{0}, 0\right)$ de coordenada esférica $\gamma_{2}$. Geometricamente temos

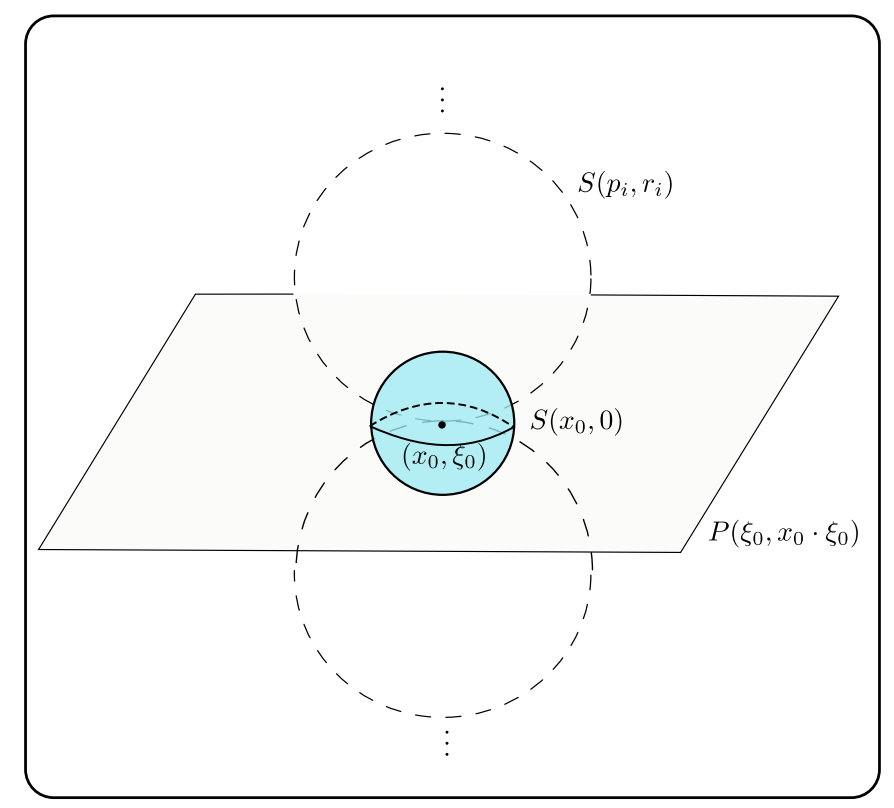

Chamaremos este conjunto de feixe de esferas orientadas. De fato, pela Proposição 2.1, escrevendo

$$
\gamma_{3}=\lambda_{3} \gamma_{1}+\mu_{3} \gamma_{2}, \gamma_{4}=\lambda_{4} \gamma_{1}+\mu_{4} \gamma_{2},
$$

temos

$$
\left\langle\gamma_{3}, \gamma_{4}\right\rangle=\left\langle\lambda_{3} \gamma_{1}+\mu_{3} \gamma_{2}, \lambda_{4} \gamma_{1}+\mu_{4} \gamma_{2}\right\rangle=0
$$


ou seja, as esferas associadas a $\gamma_{3}$ e $\gamma_{4}$ possuem também contato orientado. Dessa forma, qualquer esfera $\Gamma$ no feixe pode ser determinada mediante sua coordenada esférica $\gamma$ que pode ser escrita como

$$
\gamma=\lambda \gamma_{1}+\mu \gamma_{2} \in \mathrm{C}^{5} \backslash\{\wp\}
$$

para algum $(\lambda, \mu) \in \mathbb{R}^{2} \backslash\{(0,0)\}$. Assim, o ponto $(x, \xi) \in U \mathbb{R}^{3}$ determina uma única reta $\mathrm{C}^{5} \backslash\{\wp\}$ dada por $\left\{\lambda \gamma_{1}+\mu \gamma_{2} \mid(\lambda, \mu) \in \mathbb{R}^{2} \backslash\{(0,0)\}\right\}$, consequentemente, é determinada uma única reta projetiva em $\mathrm{Q}^{4} \backslash\{[\wp]\}$ dada por

$$
\left\{\left[\lambda \gamma_{1}+\mu \gamma_{2}\right] \mid(\lambda, \mu) \in \mathbb{R}^{2} \backslash\{(0,0)\}\right\}
$$

Usando o fato citado acima, pode-se definir o conjunto $\Lambda^{5}$ formado por todas as retas projetivas em $\mathrm{Q}^{4} \backslash\{[\wp]\}$. Assim, temos uma propriedade equivalente à Proposição 2.1.

Proposição 2.2. Sejam $\Gamma_{1}, \Gamma_{2} \in \Sigma$ com coordenadas esféricas $\gamma_{1}, \gamma_{2}$, respectivamente. Então, $\Gamma_{1}$ e $\Gamma_{2}$ possuem contato orientado se, e somente se todas as retas projetivas $\left\{\left[\lambda \gamma_{1}+\mu \gamma_{2}\right] \mid(\lambda, \mu) \in \mathbb{R}^{2} \backslash\{(0,0)\}\right\}$ estão completamente em $\mathrm{Q}^{4} \backslash\{[\wp]\}$.

Demonstração.

$\Rightarrow$ ] Suponha que $\Gamma_{1}, \Gamma_{2} \in \Sigma$ com coordenadas esféricas $\gamma_{1}, \gamma_{2}$, respectivamente, possuem contato orientado, então $\left\langle\gamma_{1}, \gamma_{2}\right\rangle=0$. Seja agora, qualquer $(\lambda, \mu) \in \mathbb{R}^{2} \backslash\{(0,0)\}$, então temos a reta projetiva

$$
\mathcal{L}=\left\{\left[\lambda \gamma_{1}+\mu \gamma_{2}\right] \mid(\lambda, \mu) \in \mathbb{R}^{2} \backslash\{(0,0)\}\right\}
$$

satisfaz

$$
\left\langle\lambda \gamma_{1}+\mu \gamma_{2}, \lambda \gamma_{1}+\mu \gamma_{2}\right\rangle=\lambda^{2}\left\langle\gamma_{1}, \gamma_{1}\right\rangle+2 \lambda \mu\left\langle\gamma_{1}, \gamma_{2}\right\rangle+\mu^{2}\left\langle\gamma_{2}, \gamma_{2}\right\rangle=0
$$

dai, temos que $\lambda \gamma_{1}+\mu \gamma_{2} \in \mathcal{C}^{5} \backslash\{\wp\}$ ou equivalentemente, que $\left[\lambda \gamma_{1}+\mu \gamma_{2}\right] \in \mathrm{Q}^{4} \backslash\{[\wp]\}$, portanto $\mathcal{L} \subset \mathrm{Q}^{4} \backslash\{[\wp]\}$.

$\Leftarrow$ ] Suponha que qualquer reta descrita em (2.24) está completamente contida em $\mathrm{Q}^{4} \backslash\{[\wp]\}$, então $\gamma_{1}$ e $\gamma_{2}$ são, respectivamente, coordenadas esféricas de duas esferas orientadas $\Gamma_{1}, \Gamma_{2} \in \Sigma$. Já que $\lambda \gamma_{1}+\mu \gamma_{2} \in \mathrm{C}^{5} \backslash\{\wp\}$, temos

$$
0=\left\langle\lambda \gamma_{1}+\mu \gamma_{2}, \lambda \gamma_{1}+\mu \gamma_{2}\right\rangle=\lambda^{2}\left\langle\gamma_{1}, \gamma_{1}\right\rangle+2 \lambda \mu\left\langle\gamma_{1}, \gamma_{2}\right\rangle+\mu^{2}\left\langle\gamma_{2}, \gamma_{2}\right\rangle=2 \lambda \mu\left\langle\gamma_{1}, \gamma_{2}\right\rangle
$$

como neste caso não trivial temos $\lambda, \mu \in \mathbb{R} \backslash\{0\}$, então $\left\langle\gamma_{1}, \gamma_{2}\right\rangle=0$, portanto $\Gamma_{1}$ e $\Gamma_{2}$ possuem contato orientado.

Definição 2.7 (Difeomorfismo de Lie). A aplicação $L: U \mathbb{R}^{3} \rightarrow \Lambda^{5}$ definida por

$$
L((x, \xi))=\left[\lambda \gamma_{1}+\mu \gamma_{2}\right] \in \mathrm{Q}^{4} \backslash\{[\wp]\},(\lambda, \mu) \in \mathbb{R}^{2} \backslash\{(0,0)\}
$$

é um difeomorfismo, chamado de difeomorfismo de Lie.

Agora, partindo do fato de que cada ponto $(x, \xi) \in U \mathbb{R}^{3}$ determina um único feixe de esferas de contato orientado em $x \in \mathbb{R}^{3}$ com o mesmo vetor normal unitário $\xi \in S^{2}$, associemos o ponto esférico 
$S(x, 0)$ e o plano $P(\xi, x \cdot \xi)$, por sua vez elas tem associadas, respectivamente, os vetores de $\mathrm{C}^{5} \backslash\{\wp\}$

$$
\begin{aligned}
\gamma_{1} & =\left(\frac{1}{2}\left(1+|x|^{2}\right), \frac{1}{2}\left(1-|x|^{2}\right), x, 0\right), \\
\gamma_{2} & =(x \cdot \xi,-x \cdot \xi, \xi, 1)
\end{aligned}
$$

então, é possível definir um difeomorfismo de Lie por

$$
\begin{aligned}
\phi: U \mathbb{R}^{3} & \rightarrow \Lambda^{5} \\
(x, \xi) & \mapsto \phi(x, \xi)=\left[\lambda\left(\frac{1}{2}\left(1+|x|^{2}\right), \frac{1}{2}\left(1-|x|^{2}\right), x, 0\right)+\mu(x \cdot \xi,-x \cdot \xi, \xi, 1)\right] .
\end{aligned}
$$

\subsection{Grupo de transformações de Laguerre sobre $U \mathbb{R}^{3}$}

Nesta seção introduzimos o conceito de transformação de Laguerre e apresentamos o grupo de transformações de Laguerre sobre $U \mathbb{R}^{3}$. O principal objetivo é gerar o grupo de transformações de Laguerre a partir de isometrias em $\mathbb{R}^{3}$, transformações parabólicas e transformações hiperbólicas em $U \mathbb{R}^{3}$.

Definição 2.8. Em $\mathbb{R}^{6}$ temos um produto escalar dado em coordenadas pela matriz diagonal $\mu=\operatorname{diag}(-1,1,1,1,1-1)$, então, uma forma quadrática é da forma

$$
Q\left(x_{1}, x_{2}, x_{3}, x_{4}, x_{5}, x_{6}\right)=-x_{1}^{2}+x_{2}^{2}+x_{3}^{2}+x_{4}^{2}+x_{5}^{2}-x_{6}^{2} .
$$

Definimos o grupo semi-ortogonal $O(4,2)$ como o subgrupo de matrizes $M$ de ordem 6 tais que

$$
Q(M v)=Q v, v \in \mathbb{R}^{6 \times 1},
$$

ou equivalentemente, como aquelas satisfazendo $M^{t} \mu M=\mu$. De forma análoga, define-se $O(3,1)$.

Definição 2.9. Seja LG o subgrupo do grupo semi-ortogonal $O(4,2)$ que fixa a coordenada esférica do ponto esférico $S(x, 0)$ no infinito de $\mathbb{R}^{3}$, em outras palavras

$$
L \mathbb{G}=\{T \in O(4,2) \mid \wp T=\wp\}
$$

Vamos determinar a dimensão de $L \mathbb{G}$, para isso, considere uma curva diferenciável $T:(-\varepsilon, \varepsilon) \rightarrow L \mathbb{G}$, com $T(0)=I_{6 \times 6}$ e chame $J=\operatorname{diag}(-1,1,1,1,1,-1)$. Já que $\forall s \in(-\varepsilon, \varepsilon), T(s) \in O(4,2)$, temos que

$$
T^{t}(s) J T(s)=J
$$

então, já que $J$ é constante,

$$
\left[\left(T^{t}(s)\right)^{\prime} J\right] T(s)+T^{t}(s) J T^{\prime}(s)=\mathbf{0}_{6 \times 6},
$$

particularmente para $s=0$, temos que

$$
\left(T^{t}(0)\right)^{\prime} J=-J T^{\prime}(0)
$$


chamemos $T^{\prime}=\left(T_{i j}\right)$, então

$$
\begin{aligned}
\left(T^{t}(0)\right)^{\prime} J=\left(T^{\prime}(0)\right)^{t} J & =\left(\begin{array}{llllll}
T_{11} & T_{21} & T_{31} & T_{41} & T_{51} & T_{61} \\
T_{12} & T_{22} & T_{32} & T_{42} & T_{52} & T_{62} \\
T_{13} & T_{23} & T_{33} & T_{43} & T_{53} & T_{63} \\
T_{14} & T_{24} & T_{34} & T_{44} & T_{54} & T_{64} \\
T_{15} & T_{25} & T_{35} & T_{45} & T_{55} & T_{65} \\
T_{16} & T_{26} & T_{36} & T_{46} & T_{56} & T_{66}
\end{array}\right)\left(\begin{array}{cccccc}
-1 & 0 & 0 & 0 & 0 & 0 \\
0 & 1 & 0 & 0 & 0 & 0 \\
0 & 0 & 1 & 0 & 0 & 0 \\
0 & 0 & 0 & 1 & 0 & 0 \\
0 & 0 & 0 & 0 & 1 & 0 \\
0 & 0 & 0 & 0 & 0 & -1
\end{array}\right) \\
& =\left(\begin{array}{llllllll}
-T_{11} & T_{21} & T_{31} & T_{41} & T_{51} & -T_{61} \\
-T_{12} & T_{22} & T_{32} & T_{42} & T_{52} & -T_{62} \\
-T_{13} & T_{23} & T_{33} & T_{43} & T_{53} & -T_{63} \\
-T_{14} & T_{24} & T_{34} & T_{44} & T_{54} & -T_{64} \\
-T_{15} & T_{25} & T_{35} & T_{45} & T_{55} & -T_{65} \\
-T_{16} & T_{26} & T_{36} & T_{46} & T_{56} & -T_{66}
\end{array}\right) .
\end{aligned}
$$

Por outro lado, temos

$$
\begin{aligned}
&-J T^{\prime}(0)=\left(\begin{array}{cccccc}
1 & 0 & 0 & 0 & 0 & 0 \\
0 & -1 & 0 & 0 & 0 & 0 \\
0 & 0 & -1 & 0 & 0 & 0 \\
0 & 0 & 0 & -1 & 0 & 0 \\
0 & 0 & 0 & 0 & -1 & 0 \\
0 & 0 & 0 & 0 & 0 & -1
\end{array}\right)\left(\begin{array}{ccccccc}
T_{11} & T_{12} & T_{13} & T_{14} & T_{15} & T_{16} \\
T_{21} & T_{22} & T_{23} & T_{24} & T_{25} & T_{26} \\
T_{31} & T_{32} & T_{33} & T_{34} & T_{35} & T_{36} \\
T_{41} & T_{42} & T_{43} & T_{44} & T_{45} & T_{46} \\
T_{51} & T_{52} & T_{53} & T_{54} & T_{55} & T_{56} \\
T_{61} & T_{62} & T_{63} & T_{64} & T_{65} & T_{66}
\end{array}\right) \\
&=\left(\begin{array}{ccccccc}
T_{11} & T_{12} & T_{13} & T_{14} & T_{15} & T_{16} \\
-T_{21} & -T_{22} & -T_{23} & -T_{24} & -T_{25} & -T_{26} \\
-T_{31} & -T_{32} & -T_{33} & -T_{34} & -T_{35} & -T_{36} \\
-T_{41} & -T_{42} & -T_{43} & -T_{44} & -T_{45} & -T_{46} \\
-T_{51} & -T_{52} & -T_{53} & -T_{54} & -T_{55} & -T_{56} \\
T_{61} & T_{62} & T_{63} & T_{64} & T_{65} & T_{66}
\end{array}\right) . \\
&
\end{aligned}
$$

Igualando (2.28) e (2.29), obtemos que

$$
\begin{array}{llllll}
T_{11}=0, & T_{21}=T_{12}, & T_{31}=T_{13}, & T_{41}=T_{14}, & T_{51}=T_{15}, & T_{61}=-T_{16}, \\
T_{12}=T_{21}, & T_{22}=0, & T_{32}=-T_{32}, & T_{42}=-T_{24}, & T_{52}=-T_{25}, & T_{62}=T_{26}, \\
T_{13}=T_{31}, & T_{23}=-T_{32}, & T_{33}=0, & T_{43}=-T_{34}, & T_{53}=-T_{35}, & T_{63}=T_{36}, \\
T_{14}=T_{41}, & T_{24}=-T_{42}, & T_{34}=-T_{43}, & T_{44}=0, & T_{54}=-T_{45}, & T_{64}=T_{46}, \\
T_{15}=T_{51}, & T_{25}=-T_{52}, & T_{35}=-T_{53}, & T_{45}=-T_{54}, & T_{55}=0, & T_{65}=T_{56}, \\
T_{16}=-T_{61}, & T_{26}=T_{62}, & T_{36}=T_{63}, & T_{46}=T_{64}, & T_{56}=T_{65}, & T_{66}=0 .
\end{array}
$$


Assim,

$$
T^{\prime}(0)=\left(\begin{array}{cccccc}
0 & T_{12} & T_{13} & T_{14} & T_{15} & T_{16} \\
T_{12} & 0 & T_{23} & T_{24} & T_{25} & T_{26} \\
T_{13} & -T_{23} & 0 & T_{34} & T_{35} & T_{36} \\
T_{14} & -T_{24} & -T_{34} & 0 & T_{45} & T_{46} \\
T_{15} & -T_{25} & -T_{35} & -T_{45} & 0 & T_{56} \\
-T_{16} & T_{26} & T_{36} & T_{46} & T_{56} & 0
\end{array}\right)
$$

Como $T^{\prime}(0) \in L \mathbb{G}$, temos que $\wp T^{\prime}(0)=\wp$, mas

$$
(1,-1,0,0,0,0)\left(\begin{array}{cccccc}
0 & T_{12} & T_{13} & T_{14} & T_{15} & T_{16} \\
T_{12} & 0 & T_{23} & T_{24} & T_{25} & T_{26} \\
T_{13} & -T_{23} & 0 & T_{34} & T_{35} & T_{36} \\
T_{14} & -T_{24} & -T_{34} & 0 & T_{45} & T_{46} \\
T_{15} & -T_{25} & -T_{35} & -T_{45} & 0 & T_{56} \\
-T_{16} & T_{26} & T_{36} & T_{46} & T_{56} & 0
\end{array}\right)^{t}=\left(\begin{array}{c}
T_{12} \\
T_{12} \\
T_{13}-T_{23} \\
T_{14}-T_{24} \\
T_{15}-T_{25} \\
T_{16}-T_{26}
\end{array}\right)^{t}
$$

dai, temos que $\left(T_{12} T_{12}, T_{13}-T_{23}, T_{14}-T_{24}, T_{15}-T_{25}, T_{16}-T_{26}\right)=(1,-1,0,0,0,0)$, e consequentemente

$$
\begin{aligned}
& T_{12}=-1, \\
& T_{13}=T_{23}, \\
& T_{14}=T_{24}, \\
& T_{15}=T_{25}, \\
& T_{16}=T_{26} .
\end{aligned}
$$

Portanto,

$$
T^{\prime}(0)=\left(\begin{array}{cccccc}
0 & -1 & T_{13} & T_{14} & T_{15} & T_{16} \\
-1 & 0 & T_{13} & T_{14} & T_{15} & T_{16} \\
T_{13} & -T_{13} & 0 & T_{34} & T_{35} & T_{36} \\
T_{14} & -T_{14} & -T_{34} & 0 & T_{45} & T_{46} \\
T_{15} & -T_{25} & -T_{35} & -T_{45} & 0 & T_{56} \\
-T_{16} & T_{16} & T_{36} & T_{46} & T_{56} & 0
\end{array}\right)
$$

O que mostra que $\operatorname{dim} L \mathbb{G}=10$.

Agora, vamos determinar as matrizes $T \in L \mathbb{G}$, para isso, chamamos $T=\left(a_{i j}\right)$ e usamos o fato $\wp=T \wp$, então

$$
(1,-1,0,0,0,0)\left(\begin{array}{llllll}
a_{11} & a_{12} & a_{13} & a_{14} & a_{15} & a_{16} \\
a_{21} & a_{22} & a_{23} & a_{24} & a_{25} & a_{26} \\
a_{31} & a_{32} & a_{33} & a_{34} & a_{35} & a_{36} \\
a_{41} & a_{42} & a_{43} & a_{44} & a_{45} & a_{46} \\
a_{51} & a_{52} & a_{53} & a_{54} & a_{55} & a_{56} \\
a_{61} & a_{62} & a_{63} & a_{64} & a_{65} & a_{16}
\end{array}\right)=\left(\begin{array}{c}
a_{11}-a_{21} \\
a_{12}-a_{22} \\
a_{13}-a_{23} \\
a_{14}-a_{24} \\
a_{15}-a_{25} \\
a_{16}-a_{26}
\end{array}\right)^{t}
$$


assim, $a_{11}-a_{21}=1, a_{12}-a_{22}=-1, a_{1 i}-a_{2 i}=0$, para $i \geq 3$. Dessa forma, $T$ é escrita como

$$
T=\left(\begin{array}{cccccc}
1+a_{21} & a_{12} & a_{13} & a_{14} & a_{15} & a_{16} \\
a_{21} & a_{12}+1 & a_{13} & a_{13} & a_{14} & a_{16} \\
a_{31} & a_{32} & a_{33} & a_{34} & a_{35} & a_{36} \\
a_{41} & a_{42} & a_{43} & a_{44} & a_{45} & a_{46} \\
a_{51} & a_{52} & a_{53} & a_{54} & a_{55} & a_{56} \\
a_{61} & a_{62} & a_{63} & a_{64} & a_{65} & a_{16}
\end{array}\right)
$$

Vamos fazer as seguintes mudanças

$$
\begin{gathered}
\mathbf{a}=\left(\begin{array}{lll}
a_{13} & a_{14} & a_{15}
\end{array}\right), \quad a_{16}=\rho . \\
\left(\begin{array}{ll}
A & u \\
v & w
\end{array}\right):=\left(\begin{array}{lll|l}
a_{33} & a_{34} & a_{35} & a_{36} \\
a_{43} & a_{44} & a_{45} & a_{46} \\
a_{53} & a_{54} & a_{55} & a_{56} \\
\hline a_{63} & a_{64} & a_{65} & a_{66}
\end{array}\right), \\
\mathbf{b}=\left(\begin{array}{l}
a_{31} \\
a_{41} \\
a_{51} \\
a_{61}
\end{array}\right) \stackrel{\text { not. }}{=}\left(b_{i}\right)_{4 \times 1}, \quad \mathbf{c}=\left(\begin{array}{l}
a_{32} \\
a_{42} \\
a_{52} \\
a_{62}
\end{array}\right) \stackrel{\text { not. }}{=}\left(c_{i}\right)_{4 \times 1},
\end{gathered}
$$

assim,

$$
T=\left(\begin{array}{cc|cc}
1+a_{21} & a_{12} & \mathbf{a} & \rho \\
a_{21} & a_{12}+1 & \mathbf{a} & \rho \\
\hline \mathbf{b} & \mathbf{c} & & \\
& & \mathbf{v} & w
\end{array}\right)
$$

Sendo as colunas e as linhas pseudo-ortonormais, usando as duas primeiras linhas temos,

$$
\begin{array}{r}
-\left(1+a_{21}\right)^{2}+a_{12}^{2}+|a|^{2}-\rho^{2}=-1 \\
-a_{21}^{2}+\left(a_{12}+1\right)^{2}+|a|^{2}-\rho^{2}=1 \\
-\left(1+a_{21}\right) a_{21}+a_{12}\left(a_{12}+1\right)+|a|^{2}-\rho^{2}=0
\end{array}
$$

equivalentemente

$$
\begin{aligned}
& -1-2 a_{21}-a_{21}^{2}+a_{12}^{2}+|a|^{2}-\rho^{2}=-1 \\
& -a_{21}^{2}+a_{12}^{2}+2 a_{12}+1+|a|^{2}-\rho^{2}=1 \\
& -a_{21}-a_{21}^{2}+a_{12}^{2}+a_{12}+|a|^{2}-\rho^{2}=0 .
\end{aligned}
$$


Subtraindo a equação (2.37) da equação (2.35) temos

$$
-2 a_{21}-a_{21}^{2}+a_{12}^{2}+a_{21}+a_{21}^{2}-a_{12}^{2}-a_{12}=0,
$$

dai,

$$
-a_{21}=a_{12}
$$

Usando o resultado acima na equação (2.37), temos que

$$
\begin{gathered}
-a_{12}^{2}+a_{12}^{2}+2 a_{12}+1+|a|^{2}-\rho^{2}=1 \\
a_{12}=\frac{\rho^{2}-|a|^{2}}{2}
\end{gathered}
$$

então a matriz $T$ tem a forma

$$
T=\left(\begin{array}{cc|cc}
1+\frac{|a|^{2}}{2}-\frac{\rho^{2}}{2} & -\frac{|a|^{2}}{2}+\frac{\rho^{2}}{2} & \mathbf{a} & \rho \\
\frac{|a|^{2}}{2}-\frac{\rho^{2}}{2} & 1-\frac{|a|^{2}}{2}+\frac{\rho^{2}}{2} & \mathbf{a} & \rho \\
\hline \mathbf{b} & \mathbf{c} & A & \mathbf{u} \\
& & \mathbf{v} & w
\end{array}\right)
$$

Sendo que $\left\langle e_{i} T, \wp\right\rangle=\left\langle e_{i} T, \wp T\right\rangle=\left\langle e_{i}, \wp\right\rangle, 1 \leq i \leq 6$, temos para $i \geq 3$,

$$
e_{i} T=\left(\begin{array}{llll}
b_{i} & c_{i} & A_{i} & \mathbf{u}
\end{array}\right)
$$

onde $A_{i}$ é a i-ésima linha da matriz $A$. Logo,

$$
\begin{aligned}
\left\langle e_{i} T, \wp\right\rangle & =-b_{i}-c_{i}, \\
\left\langle e_{i}, \wp\right\rangle & =0,
\end{aligned}
$$

então $b_{i}=-c_{i}$, concluímos que $b=-c$. Agora, para $i \geq 3$ a i-ésima linha de $T$ é dada por

$$
\left(\begin{array}{llll}
b_{i} & -b_{i} & A_{i} & \mathbf{u}
\end{array}\right)
$$

Multiplicando-lhe pela primeira linha temos

$$
-b_{i}\left(1+\frac{|a|^{2}}{2}-\frac{\rho^{2}}{2}\right)-b_{i}\left(-\frac{|a|^{2}}{2}+\frac{\rho^{2}}{2}\right)+A_{i} \mathbf{a}-\rho \mathbf{u}=0
$$

assim,

$$
-\mathbf{b}+A_{i} \mathbf{a}-\rho \mathbf{u}=0,
$$

juntando as 3 equações para $i=1,2,3$, temos

$$
\mathbf{b}=A \mathbf{a}^{t}-\rho \mathbf{u} .
$$


Finalmente, $T$ é expressa como

$$
T=\left(\begin{array}{cccc}
1+|a|^{2} / 2-\rho^{2} / 2 & -|a|^{2} / 2+\rho^{2} / 2 & a & \rho \\
|a|^{2} / 2-\rho^{2} / 2 & 1+|a|^{2} / 2-\rho^{2} / 2 & a & \rho \\
A a^{\prime}-\rho u & -A a^{\prime}+\rho u & A & u \\
v a^{\prime}-\rho w & -v a^{\prime}+\rho w & v & w
\end{array}\right)
$$

para alguma matriz

$$
\left(\begin{array}{ll}
A & u \\
v & w
\end{array}\right) \in O(3,1),(a, \rho) \in \mathbb{R}^{4}, w \in \mathbb{R} .
$$

Chamamos ambas, $T \in L \mathbb{G}$ e $T: \mathrm{Q}^{4} \rightarrow \mathrm{Q}^{4}$, de transformação de Laguerre.

Sejam $\Gamma_{1}, \Gamma_{2} \in \Sigma$ duas esferas ou planos orientados com contato orientado com coordenadas associadas $\gamma_{1}$ e $\gamma_{2}$, respectivamente. Então $\left[\gamma_{1}\right]$ e $\left[\gamma_{2}\right]$ definem uma reta projetiva em $Q^{4} \backslash\{[\wp]\}$ por

$$
\operatorname{span}\left\{\left[\gamma_{1}\right],\left[\gamma_{2}\right]\right\}=\left\{\left[\lambda \gamma+\mu \gamma_{2}\right] \mid(\lambda, \mu) \in \mathbb{R}^{2} \backslash\{(0,0)\}\right\} \in \Lambda^{5}
$$

Como para qualquer $\gamma \in \mathrm{C}^{5}$ temos $T[\gamma]=[\gamma T]$, então qualquer $T \in L \mathbb{G}$ define uma transformação $T: \Lambda^{5} \rightarrow \Lambda^{5}$ por

$$
T\left(\operatorname{span}\left\{\left[\gamma_{1}\right],\left[\gamma_{2}\right]\right\}\right)=\operatorname{span}\left\{\left[\gamma_{1} T\right],\left[\gamma_{2} T\right]\right\} .
$$

Definição 2.10. Seja $L: U \mathbb{R}^{3} \rightarrow \Lambda^{5}$ o difeomorfismo de Lie. Então qualquer $T \in$ LG induz uma transformação

$$
\sigma:=L^{-1} \circ T \circ L: U \mathbb{R}^{3} \rightarrow U \mathbb{R}^{3}
$$

chamada uma transformação de Laguerre sobre $U \mathbb{R}^{3}$. Assim, o grupo de transformações de Laguerre sobre $U \mathbb{R}^{3}$ é o grupo 10 - dimensional dado por

$$
L \mathbb{G}=\left\{\sigma: U \mathbb{R}^{3} \rightarrow U \mathbb{R}^{3} \mid \sigma=L^{-1} \circ T \circ L, T \in O(4,2), \wp T=\wp\right\}
$$

Seja $T \in L \mathbb{G}$ uma transformação de Laguerre, então $T: \mathrm{Q}^{4} \rightarrow \mathrm{Q} \operatorname{com} \wp T=\wp$. Já que qualquer esfera $\Gamma \in \Sigma$ determina de forma biunívoca uma coordenada $\gamma \in \mathrm{C}^{5} \backslash\{\wp\}$ temos que

$$
\langle\gamma T, \wp\rangle=\langle\gamma T, \wp T\rangle=\left\langle\gamma, T^{t} \wp T\right\rangle=\langle\gamma, \wp\rangle
$$

Logo, no caso de ser $\Gamma$ um plano orientado (respectivamente, uma esfera orientada), da Propriedade 2.1 sabemos que $\gamma T$ é coordenada de um plano orientado (respectivamente, de uma esfera orientada) . Consequentemente, temos a seguinte propriedade.

Propriedade 2.2. Toda transformação de Laguerre $\sigma: U \mathbb{R}^{3} \rightarrow U \mathbb{R}^{3}$ preserva esferas e planos orientados.

Exemplo 2.1. Seja $A \in O(3), a \in \mathbb{R}^{3}$ então a isometria $\sigma(x)=x A+a$, induz uma transformação isométrica $\sigma: U \mathbb{R}^{3} \rightarrow U \mathbb{R}^{3}$ definida por

$$
\sigma((x, \xi))=(x A+a, \xi A)
$$

De fato, para verificar que $\sigma \in \mathbb{L} G$, deve-se verificar que $\sigma=L^{-1} \circ T \circ L$, onde $T \in G$ e $L$ é um 
difeomorfismo de Lie, para isso, escolha

$$
T=\left(\begin{array}{cccc}
1+\frac{|a|^{2}}{2} & -\frac{|a|^{2}}{2} & a & 0 \\
\frac{|a|^{2}}{2} & 1-\frac{|a|^{2}}{2} & a & 0 \\
A a^{t} & -A a^{t} & A & 0 \\
0 & 0 & 0 & 1
\end{array}\right) \in L \mathbb{G}
$$

equivalentemente, provaremos que $L \circ \sigma=T \circ L$ e usaremos o difeomorfismo de Lie $L$ da expressão (2.27). Assim, para cada $(x, \xi) \in U \mathbb{R}^{3}$ temos

$$
\begin{aligned}
\gamma_{1} & =\left(\frac{1}{2}\left(1+|x|^{2}\right), \frac{1}{2}\left(1-|x|^{2}\right), x, 0\right), \\
\gamma_{2} & =(x \cdot \xi,-x \cdot \xi, \xi, 1)
\end{aligned}
$$

e

$$
\begin{aligned}
T \circ L(x, \xi) & =L(x, \xi) T \\
& =\left[\lambda \gamma_{1}+\mu \gamma_{2}\right] T \\
& =\left[\left(\frac{\lambda}{2}\left(1+|x|^{2}\right)+\mu x \cdot \xi, \frac{\lambda}{2}\left(1-|x|^{2}\right)-\mu x \cdot \xi, \lambda x+\mu \xi, \mu\right) T\right] \\
& =\left[\left(c_{1}, c_{2}, C, c_{3}\right)\right],
\end{aligned}
$$

onde as entradas são

$$
\begin{aligned}
c_{1} & =\frac{\lambda}{2}\left(1+|x|^{2}\right)+\mu x \cdot \xi+\frac{|a|^{2}}{2}\left(\frac{\lambda}{2}\left(1+|x|^{2}\right)+\mu x \cdot \xi\right)+\frac{|a|^{2}}{2}\left(\frac{\lambda}{2}\left(1-|x|^{2}\right)-\mu x \cdot \xi\right)+(\lambda x+\mu \xi) A a^{\prime} \\
& =\frac{\lambda}{2}\left(1+|x|^{2}\right)+\mu x \cdot \xi+\frac{\lambda|a|^{2}}{2}+\lambda x A a^{t}+\mu \xi A a^{t} \\
& =\frac{\lambda}{2}\left(1+|x|^{2}\right)+\frac{\lambda}{2}|a|^{2}+\mu\left(x \cdot \xi+\xi A a^{t}\right)+\lambda x A a^{t}, \\
c_{2} & =-\frac{|a|^{2}}{2}\left(\frac{\lambda}{2}\left(1+|x|^{2}\right)+\mu x \cdot \xi\right)+\frac{\lambda}{2}\left(1-|x|^{2}\right)-\mu x \cdot \xi-\frac{|a|^{2}}{2}\left(\frac{\lambda}{2}\left(1-|x|^{2}\right)-\mu x \cdot \xi\right)-(\lambda x+\mu \xi) A a^{t} \\
& =-\frac{\lambda}{2}|a|^{2}+\frac{\lambda}{2}\left(1-|x|^{2}\right)-\mu x \xi-\lambda x A a^{t}-\mu \xi A a^{t} \\
& =\frac{\lambda}{2}\left(1-|x|^{2}\right)-\frac{\lambda}{2}|a|^{2}-\mu\left(x \cdot \xi+\xi A a^{t}\right)-\lambda x A a^{t} \\
C & =a\left(\frac{\lambda}{2}\left(1+|x|^{2}\right)+\mu x \cdot \xi\right)+a\left(\frac{\lambda}{2}\left(1-|x|^{2}\right)-\mu x \cdot \xi\right)+A(\lambda x+\mu \xi)^{t} \\
& =\lambda a+A(\lambda x+\mu \xi)^{t} \\
c_{3} & =\mu
\end{aligned}
$$

Por outro lado, $L \circ \sigma(x, \xi)=L(x A+b, \xi A)$, fazendo a mudança

$$
\begin{aligned}
& x^{\prime}=x A+a, \\
& \xi^{\prime}=\xi A,
\end{aligned}
$$


temos que,

$$
\begin{aligned}
L\left(x^{\prime}, \xi^{\prime}\right)= & {\left[\left(\frac{\lambda}{2}\left(1+\left|x^{\prime}\right|^{2}\right)+\mu\left(x^{\prime} \cdot \xi^{\prime}\right), \frac{\lambda}{2}\left(1-\left|x^{\prime}\right|^{2}\right)-\mu\left(x^{\prime} \cdot \xi^{\prime}\right), \lambda x^{\prime}+\mu \xi^{\prime}, \mu\right)\right] } \\
& =\left[\left(\tilde{c}_{1}, \tilde{c}_{2}, \tilde{C}, \tilde{c}_{3}\right)\right]
\end{aligned}
$$

onde as entradas são

$$
\begin{aligned}
\tilde{c}_{1}= & \frac{\lambda}{2}(1+(x A+a) \cdot(x A+a))+\mu((x A+a) \cdot(\xi A)) \\
= & \frac{\lambda}{2}\left(1+|x|^{2}+x A a^{t}+a(x A)+|a|^{2}\right)+\mu(x \cdot \xi+a(\xi A)) \\
= & \frac{\lambda}{2}\left(1+|x|^{2}+2 x A a^{t}+|a|^{2}\right)+\mu\left(x \cdot \xi+\xi A a^{t}\right) \\
= & \frac{\lambda}{2}\left(1+|x|^{2}\right)+\lambda x A a^{t}+\frac{\lambda}{2}|a|^{2}+\mu\left(x \cdot \xi+\xi A a^{t}\right), \\
\tilde{c}_{2} & =\frac{\lambda}{2}(1-(x A+a) \cdot(x A+a))-\mu((x A+a) \cdot(\xi A)) \\
& =\frac{\lambda}{2}\left(1-|x|^{2}-2 x A a^{t}-|a|^{2}\right)-\mu\left(x \cdot \xi+\xi A a^{t}\right) \\
& =\frac{\lambda}{2}\left(1-|x|^{2}\right)-\lambda x A a^{t}-\frac{\lambda}{2}|a|^{2}-\mu\left(x \cdot \xi+\xi A a^{t}\right), \\
\tilde{C} & =\lambda(x A+a)+\mu(\xi A) \\
& =\lambda a+\lambda x A+\mu \xi A \\
& =\lambda a+(\lambda x+\mu \xi) A \\
& =\lambda a+A(\lambda x+\mu \xi)^{t}, \\
\tilde{c} 3 & =\mu .
\end{aligned}
$$

Já que $c_{1}=\tilde{c}_{1}, c_{2}=\tilde{c}_{2}, C=\tilde{C}$ e $c_{3}=\tilde{c}_{3}$, concluímos que $\sigma=L^{-1} \circ T \circ L$.

Exemplo 2.2. As transformações parabólicas uniparamétricas definidas por

$$
\phi_{t}(x, \xi)=(x+t \xi, \xi), t \in \mathbb{R}
$$

são transformações de Laguerre em $U \mathbb{R}^{3}$. Neste caso, a escolha sería

$$
T=\left(\begin{array}{cccc}
1-\frac{t^{2}}{2} & -\frac{t^{2}}{2} & 0 & -t \\
\frac{-t^{2}}{2} & 1+\frac{t^{2}}{2} & 0 & -t \\
0 & 0 & 0 & I_{3} \\
t & -t & 0 & 1
\end{array}\right) \in L \mathbb{G}
$$

Exemplo 2.3. O terceiro exemplo de tranformação de Laguerre em $U \mathbb{R}^{3}$ é a seguinte transformação hiperbólica uniparamétrica. Para qualquer $(x, \xi) \in U \mathbb{R}^{3}$ escrevemos

$$
x=\left(x_{0}, x_{1}\right) \in \mathbb{R}^{2} \times \mathbb{R}, \xi=\left(\xi_{0}, \xi_{1}\right) \in \mathbb{R}^{2} \times \mathbb{R}
$$


então uma tranformação hiperbólica

$$
\psi_{t}(x, \xi)=(x(t), \xi(t)) \in U \mathbb{R}^{3}, t \in \mathbb{R}
$$

é definida por

$$
\begin{aligned}
& x(t)=\left(x_{0}-\frac{\sinh t x_{1}}{\sinh t \xi_{1}+\cosh t} \xi_{0}, \frac{x_{1}}{\sinh t \xi_{1}+\cosh t}\right), \\
& \xi(t)=\left(\frac{1}{\sinh t \xi_{1}+\cosh t} \xi_{0}, \frac{\cosh t \xi_{1}+\sinh t}{\sinh t \xi_{1}+\cosh t}\right) .
\end{aligned}
$$

Neste caso a escolha de $T$ sería

$$
T\left(\psi_{1}\right)=L \circ \psi_{t} \circ L^{-1}=\left(\begin{array}{ccc}
I_{4} & 0 & 0 \\
0 & \cosh t & \sinh t \\
0 & \sinh t & \sinh t
\end{array}\right) \in L \mathbb{G} .
$$

Agora, sejam $\gamma_{1}, \gamma_{2}$ as coordenadas esféricas das esferas orientadas $S(p, r), S\left(p^{*}, r^{*}\right)$ em $\mathbb{R}^{3}$, respectivamente. Seja $T$ uma transformação de Laguerre dada por (2.38). Desde que

$$
\begin{gathered}
\gamma_{1}=\left(\frac{1}{2}\left(1+|p|^{2}-r^{2}\right), \frac{1}{2}\left(1-|p|^{2}+r^{2}\right), p,-r\right), \\
\gamma_{2}=\left(\frac{1}{2}\left(1+\left|p^{*}\right|^{2}-r^{* 2}\right), \frac{1}{2}\left(1-\left|p^{*}\right|^{2}+r^{* 2}\right), p^{*},-r^{*}\right),
\end{gathered}
$$

então obtemos as esferas orientadas $S(\tilde{p}, \tilde{r})$ e $S\left(\tilde{p}^{*}, \tilde{r}^{*}\right)$ associadas às coordenadas esféricas $\gamma_{1} T$ e $\gamma_{2} T$, além disso

$$
\begin{gathered}
(\tilde{p},-\tilde{r})=(p A-r v+a, p u+r w+\rho), \\
\left(\tilde{p}^{*},-\tilde{r}^{*}\right)=\left(p^{*} A-r^{*} v+a, p^{*} u+r^{*} w+\rho\right) .
\end{gathered}
$$

Logo temos

$$
\left(\tilde{p}^{*}-\tilde{p},-\tilde{r}^{*}+\tilde{r}\right)=\left(p^{*}-p,-r^{*}+r\right)\left(\begin{array}{cc}
A & u \\
v & w
\end{array}\right) .
$$

De onde segue que

$$
F=\left|p^{*}-p\right|^{2}-\left(r^{*}-r\right)^{2}
$$

é uma invariante de Laguerre. Geometricamente, se uma esfera não está contida em uma outra, então $F$ é exatamente o quadrado do segmento de tangente comum das duas esferas $S(p, r)$ e $S\left(p^{*}, r^{*}\right)$.

Teorema 2.1. Para qualquer $T \in O(4,2)$ com $\wp T=T$ existem duas isometrias $\sigma_{1}, \sigma_{2}$ sobre $U \mathbb{R}^{3}$ e constantes $s, t \in \mathbb{R}, \varepsilon= \pm 1$ tal que

$$
T=\varepsilon T\left(\sigma_{2}\right) T\left(\psi_{t}\right) T\left(\phi_{s}\right) T\left(\sigma_{1}\right)
$$

Demonstração. Ver [12].

Corolário 2.1. Qualquer transformação de Laguerre em $U \mathbb{R}^{3}$ é gerada pelas isometrias, as transformações paralelas e as transformações hiperbólicas. 


\subsection{Superfícies de Laguerre em $U \mathbb{R}^{3}$}

Nesta seção introduzimos o conceito de superfície de Laguerre e apresentamos um teorema sobre superfícies equivalentes. A partir destes conceitos, apresentamos a métrica de Laguerre e o seu elemento de volume.

Seja $(x, \xi): U \mathbb{R}^{3} \rightarrow \mathbb{R}^{3} \times S^{2} \subset \mathbb{R}^{6}$ a imersão usual. Definimos $\gamma_{1}, \gamma_{2}: U \mathbb{R}^{3} \rightarrow \mathbb{R}_{2}^{6}$ como na equação (2.26). Seja $T \in L \mathbb{G}$ uma transformação de Laguerre e

$$
(\tilde{x}, \tilde{\xi})=\phi((x, \xi)), \phi=L^{-1} \circ T \circ L: U \mathbb{R}^{3} \rightarrow U \mathbb{R}^{3} .
$$

Denotemos por $a, b$ as últimas coordenadas de $\gamma_{1} T$ e $\gamma_{2} T$, respectivamente. Logo por (2.26) e (2.38) podemos escrever

$$
\begin{gathered}
\tilde{\gamma}_{1}=\left(\frac{1}{2}\left(1+|\tilde{x}|^{2}\right), \frac{1}{2}\left(1-|\tilde{x}|^{2}\right), \tilde{x}, 0\right)=\gamma_{1} T-\frac{a}{b} \gamma_{2} T, \\
\tilde{\gamma}_{2}=(\tilde{x} \cdot \tilde{\xi},-\tilde{x} \cdot \tilde{\xi}, \tilde{\xi}, 1)=\frac{1}{b} \gamma_{2} T .
\end{gathered}
$$

Assim, segue que

$$
\begin{gathered}
d \tilde{x} \cdot \tilde{\xi}=\left\langle d \tilde{\gamma}_{1}, \tilde{\gamma}_{2}\right\rangle=\left\langle d\left(\gamma_{1} T-\frac{a}{b} \gamma_{2} T\right), \frac{1}{b} \gamma_{2} T\right\rangle=\frac{1}{b}\left\langle d \gamma_{1}, \gamma_{2}\right\rangle=\frac{1}{b} d x \cdot \xi \\
d \tilde{\xi} \cdot d \tilde{\xi}=\left\langle d \tilde{\gamma}_{2}, d \tilde{\gamma}_{2}\right\rangle=\frac{1}{b^{2}}\left\langle d \gamma_{2}, d \gamma_{2}\right\rangle=\frac{1}{b^{2}} d \xi \cdot d \xi
\end{gathered}
$$

Chamamos $f=(x, \xi): M^{2} \rightarrow U \mathbb{R}^{3}$ uma superfície de Laguerre, se $\xi: M^{2} \rightarrow \mathbb{R}^{3}$ é uma imersão e $d x \cdot \xi=0$. Segue das equações (2.49) e (2.50) que qualquer transformação de Laguerre leva superfícies de Laguerre em $U \mathbb{R}^{3}$ em superfícies de Laguerre em $U \mathbb{R}^{3}$. Pelas equações (2.1) e (2.2) sabemos que esferas orientadas e planos orientados são o caso mais simples de superfícies de Laguerre em $U \mathbb{R}^{3}$.

Seja $x: M^{2} \rightarrow \mathbb{R}^{3}$ uma superfície orientada em $\mathbb{R}^{3}$ com curvaturas principais não nulas. Então, a diferencial $d \xi$ do normal unitário unitário $\xi: M^{2} \rightarrow \mathbb{R}^{3}$ é injetiva e $\xi$ uma imersão. Logo, $x$ induz únicamente uma superfície de Laguerre $f=(x, \xi): M^{2} \rightarrow U \mathbb{R}^{3}$. Notemos que para uma superfície de Laguerre $f=(x, \xi): M^{2} \rightarrow U \mathbb{R}^{3}, x: M^{2} \rightarrow \mathbb{R}^{3}$ poderia não ser uma imersão. Pelo teorema de Pinkall (ver [19]) sabemos que a transformação paralela $f_{t}=(x+t \xi, \xi)$ de $f$ é uma imersão em qualquer ponto $p \in M^{2}$ para quase todo $t \in \mathbb{R}$. Neste sentido, a menos de uma superfície paralela, podemos supor que $x: M^{2} \rightarrow \mathbb{R}^{3}$ é uma imersão.

Definição 2.11. Sejam $x, \tilde{x}: M^{2} \rightarrow \mathbb{R}^{3}$ duas superfícies orientadas com curvaturas principais não nulas. Dizemos que $x$ e $\tilde{x}$ são Laguerre equivalentes, se as correspondentes superfícies de Laguerre $f=(x, \xi) e$ $\tilde{f}=(\tilde{x}, \tilde{\xi}): M^{2} \rightarrow U \mathbb{R}^{3}$ somente se diferenciam por uma transformação de Laguerre $\phi: U \mathbb{R}^{3} \rightarrow U \mathbb{R}^{3}$, isto é, $\tilde{f}=\phi \circ f$.

Na geometria diferencial de Laguerre estudamos propriedades das superfícies de Laguerre em $U \mathbb{R}^{3}$ que são invariantes pelo grupo de transformações de Laguerre em $U \mathbb{R}^{3}$. 
A seguir fornecemos um critério para que superfícies orientadas sejam Laguerre equivalentes. Considere a classe da coordenada de cada plano tangente em $x$ dada por

$$
[y]: M \rightarrow Q^{4}, \quad y=(x, \cdot \xi,-x \cdot \xi, \xi, 1)
$$

Teorema 2.2. Seja $x, x^{*}: M \rightarrow \mathbb{R}^{3}$ duas superfícies orientadas com curvatura principal não nula. Então $x$ e $x^{*}$ são Laguerre equivalentes se, e somente se, existe $T \in L \mathbb{G}$ tal que $\left[y^{*}\right]=[y T]$.

Demonstração. Sejam $\xi$ e $\xi^{*}$ as normais unitárias de $x$ e $x^{*}$, respectivamente. Se existe uma transformação de Laguerre $\phi=L^{-1} \circ T \circ L \in L \mathbb{G}$ tal que $\left(x^{*}, \xi^{*}\right)=\phi \circ(x, \xi)$, logo por (2.48) obtemos que $\left[\gamma^{*}\right]=[\gamma T]$. Reciprocamente, se $\left[\gamma^{*}\right]=[\gamma T]$ para algum $T \in L \mathbb{G}$, definimos $(\tilde{x}, \tilde{\xi})=\phi \circ(x, \xi) \operatorname{com} \phi=L^{-1} \circ T \circ L$. Logo por (2.48) temos que $[\tilde{\gamma}]=[\gamma T]=\left[\gamma^{*}\right]$. Segue que

$$
(\tilde{x} \cdot \tilde{\xi},-\tilde{x} \cdot \tilde{\xi}, \tilde{\xi}, 1)=\left(x^{*} \cdot \xi^{*},-x^{*} \cdot \xi^{*}, \xi^{*}, 1\right) .
$$

Seja $\left\{e_{1}, e_{2}, e_{3}\right\}$ uma base local para $T M$. Desde que $\xi^{*}: M \rightarrow \mathbb{R}^{3}$ é uma imersão, sabemos que $\left\{e_{1}\left(\xi^{*}\right), e_{2}\left(\xi^{*}\right), \xi^{*}\right\}$ é uma base para $\mathbb{R}^{3}$. De $(2.52)$ e do fato que

$$
\xi^{*}=\tilde{\xi},\left(x^{*}-\tilde{x}\right) \cdot \xi^{*}=0,\left(x^{*}-\tilde{x}\right) \cdot d \xi^{*}=d\left(\left(x^{*}-\tilde{x}\right) \cdot \xi^{*}\right)=0,
$$

obtemos $x^{*}=\tilde{x}$. Então temos que $\left(x^{*}, \xi^{*}\right)=\phi \circ(x, \xi)$, o que implica que $x$ e $x^{*}$ são Laguerre equivalentes.

Por (2.51) temos que $\langle d y, d y\rangle=d \xi \cdot d \xi$, o que é exatamente a terceira forma fundamental de $x$. Segue do Teorema 2.2 o seguinte corolário.

Corolário 2.2. A classe conforme da terceira forma fundamental de uma superfície $x: M \rightarrow \mathbb{R}^{3}$ é uma invariante de Laguerre.

Seja $x: M \rightarrow \mathbb{R}^{3}$ uma superfície orientada com curvaturas principais não nulas. Seja $I I I=\langle d y, d y\rangle$ a terceira forma fundamental de $x$. Para qualquer base ortonormal $\left\{E_{1}, E_{2}\right\}$ em relação à $I I I$ definimos

$$
\mathbb{V}=\operatorname{span}\left\{y, \Delta^{I I I} y, E_{1}(y), E_{2}(y)\right\},
$$

onde $\Delta^{I I I}$ é o operador Laplaciano em relação à $I I I=\langle d y, d y\rangle$. Observe que do fato $\langle y, y\rangle$ temos que $\left\langle E_{i}(y), y\right\rangle=0$. Além disso, da ortonormalidade da base $\left\{E_{1}, E_{2}\right\}$, temos que $\left\langle E_{j}(y), E_{j}(y)\right\rangle=1$, então

$$
\left\langle E_{i}\left(E_{j}(y)\right), E_{j}(y)\right\rangle=0
$$

por outro lado, temos também que $\left\langle E_{i}(y), E_{j}(y)\right\rangle=0$, então

$$
\left\langle E_{i}\left(E_{i}(y)\right), E_{j}(y)\right\rangle+\left\langle E_{i}(y), E_{i}\left(E_{j}(y)\right)\right\rangle \stackrel{(2.54)}{=}\left\langle E_{i}\left(E_{i}(y)\right), E_{j}(y)\right\rangle=0,
$$

logo temos

$$
\begin{aligned}
\sum_{i}\left\langle E_{i}\left(E_{i}(y)\right), E_{j}(y)\right\rangle & =\left\langle\sum_{i} E_{i}\left(E_{i}(y)\right), E_{j}(y)\right\rangle \\
& =\left\langle\Delta^{I I I} y, E_{j}(y)\right\rangle \\
& =0 .
\end{aligned}
$$


Agora, como $\left\langle E_{i}(y), y\right\rangle=0$, temos

$$
\left\langle E_{i}\left(E_{i}(y)\right), y\right\rangle+\left\langle E_{i}(y), E_{i}(y)\right\rangle=0,
$$

ou seja, $\left\langle E_{i}\left(E_{i}(y)\right), y\right\rangle=-1$, assim

$$
\left\langle\Delta^{I I I} y, y\right\rangle=-2 .
$$

Em conclusão, temos as seguintes equações

$$
\begin{aligned}
\left\langle y, E_{i}(y)\right\rangle=\left\langle\Delta^{I I I} y, E_{i}(y)\right\rangle & =0, \\
\left\langle\Delta^{I I I} y, y\right\rangle & =-2, \\
\left\langle E_{i}(y), E_{j}(y)\right\rangle & =\delta_{i j} .
\end{aligned}
$$

Assim, em cada ponto do espaço $\mathbb{V}$ temos um subespaço 4 - dimensional não degenerado de $\mathbb{R}_{2}^{6}$ do tipo $(-,+,+,+)$. Seja

$$
\mathbb{R}_{2}^{6}=\mathbb{V} \oplus \mathbb{V}^{\perp}=\operatorname{span}\left\{y, \Delta y, E_{1}(y), E_{2}(y)\right\} \oplus \mathbb{V}^{\perp}
$$

a descomposição ortogonal de $\mathbb{R}_{2}^{6}$. Então $\mathbb{V}^{\perp}$ é um subespaço 2 -dimensional não degenerado de $\mathbb{R}_{2}^{6}$ do tipo $(-,+)$.

Seja $\left\{e_{1}, e_{2}\right\}$ a base ortonormal de $T M$ em relação à $d x \cdot d x$, consistindo de vetores principais unitários. Escrevemos as equações de estrutura de $x: M \rightarrow \mathbb{R}^{3}$ por

$$
e_{j}\left(e_{i}(x)\right)=\sum_{k} \Gamma_{i j}^{k} e_{k}(x)+k_{i} \delta_{i j} \xi, e_{i}(\xi)=-k_{i} e_{i}(x), 1 \leq i, j, k \leq 2
$$

onde $k_{i} \neq 0$ é a curvatura principal correspondente a $e_{i}$. Observe que a segunda equação equivale a $d \xi\left(e_{i}\right)=-k_{i} e_{i}$. Seja

$$
r_{i}=\frac{1}{k_{i}}, r=\frac{r_{1}+r_{2}}{2}=\frac{H}{K}
$$

o raio de curvatura e a média dos raios de curvatura de $x$. Então a esfera da média dos raios de curvatura $S(x+r \xi,-r)$ de $x$ em $\mathbb{R}^{3}$ tem coordenada esférica $\eta$, dada a partir da equação (2.1) por

$$
\begin{aligned}
\eta & =\left(\frac{1}{2}\left(1+|x+r \xi|^{2}-r^{2}\right), \frac{1}{2}\left(1-|x-r \xi|^{2}+r^{2}\right), x+r \xi, r\right) \\
& =\left(\frac{1}{2}\left(1+|x|^{2}+2 r x \cdot \xi+r^{2}|\xi|^{2}-r^{2}\right), \frac{1}{2}\left(1-|x|^{2}-2 r x \cdot \xi-r^{2}|\xi|^{2}+r^{2}\right), x+r \xi, r\right) \\
& =\left(\frac{1}{2}\left(1+|x|^{2}+2 r x \cdot \xi\right), \frac{1}{2}\left(1-|x|^{2}-2 r x \cdot \xi\right), x+r \xi, r\right) \\
& =\left(\frac{1}{2}\left(1+|x|^{2}\right), \frac{1}{2}\left(1-|x|^{2}\right), x, 0\right)+r(x \cdot \xi,-x \cdot \xi, \xi, 1) .
\end{aligned}
$$

Definimos $E_{i}=r_{i} e_{i}, 1 \leq i \leq 2, \operatorname{assim}\left\{E_{1}, E_{2}\right\}$ é uma base ortonormal para $I I I=\langle d y, d y\rangle=d \xi \cdot d \xi$. 
Observe que

$$
\begin{aligned}
I I I\left(E_{i}, E_{j}\right) & =\left\langle d \xi\left(E_{i}\right), d \xi\left(E_{j}\right)\right\rangle \\
& =\left\langle r_{i} d \xi\left(e_{i}\right), r_{j} d \xi\left(e_{j}\right)\right\rangle \\
& =\left\langle\frac{r_{i}}{r_{i}} e_{i}, \frac{r_{j}}{r_{j}} e_{i}\right\rangle \\
& =\left\langle e_{i}, e_{j}\right\rangle
\end{aligned}
$$

e também

$$
\begin{aligned}
e_{i}(x) \cdot \xi & =-r_{i} e_{i}(\xi) \cdot \xi \\
& =-\frac{r_{i}}{2}\left(e_{i}(\xi \cdot \xi)\right) \\
& =0
\end{aligned}
$$

Das equações de estrutura em (2.57) obtemos

$$
\begin{aligned}
E_{i}(y)=r_{i} e_{i}(y) & =r_{i}\left(e_{i}(x) \cdot \xi+x \cdot e_{i}(\xi),-e_{i}(x) \cdot \xi-x \cdot e_{i}(\xi), e_{i}(\xi), 0\right) \\
\stackrel{(2.60)}{=} & r_{i}\left(x \cdot e_{i}(\xi),-x \cdot e_{i}(\xi), e_{i}(\xi), 0\right) \\
& =r_{i} k_{i}\left(-x \cdot e_{i}(x), x \cdot e_{i}(x),-e_{i}(x), 0\right) \\
& =-\left(x \cdot e_{i}(x),-x \cdot e_{i}(x), e_{i}(x), 0\right) .
\end{aligned}
$$

Por outra parte, como $\eta=\left(\frac{1}{2}\left(1+|x|^{2}\right), \frac{1}{2}\left(1-|x|^{2}\right), x, 0\right)+r y$, temos

$$
\begin{aligned}
\langle y, \eta\rangle= & \left\langle(x \cdot \xi,-x \cdot \xi, \xi, 1),\left(\frac{1}{2}\left(1+|x|^{2}\right), \frac{1}{2}\left(1-|x|^{2}\right), x, 0\right)\right\rangle+r\langle y, y\rangle \\
= & -\frac{1}{2}(x \cdot \xi)\left(1+|x|^{2}\right)-\frac{1}{2}(x \cdot \xi)\left(1-|x|^{2}\right)+x \cdot \xi \\
= & -x \cdot \xi+x \cdot \xi \\
= & 0 \\
\left\langle E_{i}, \eta\right\rangle= & -\left\langle\left(x \cdot e_{i}(x),-x \cdot e_{i}(x), e_{i}(x), 0\right),\left(\frac{1}{2}\left(1+|x|^{2}\right), \frac{1}{2}\left(1-|x|^{2}\right), x, 0\right)\right\rangle \\
& -r\left\langle\left(x \cdot e_{i}(x),-x \cdot e_{i}(x), e_{i}(x), 0\right),(x \cdot \xi,-x \cdot \xi, \xi, 1)\right\rangle \\
= & -(-x \cdot \xi+x \cdot \xi)-r\left(-\left(x \cdot e_{i}(x)\right)(x \cdot \xi)+\left(x \cdot e_{i}(x)\right)(x \cdot \xi)+e_{i}(x) \xi\right) \\
\stackrel{(2.60)}{=} & 0, \quad \begin{array}{l}
= \\
=
\end{array} \\
E_{i}(\eta)= & r_{i} e_{i}(\eta)=r_{i}\left(\frac{1}{2}\left(2 e_{i}(x) \cdot x\right),-\frac{1}{2}\left(2 e_{i}(x) \cdot x\right), e_{i}(x), 0\right)+r_{i}(r) y+r_{i} e_{i}(y) y+r E_{i}(y) \\
= &
\end{aligned}
$$


Assim, das equações (2.62), (2.63) e (2.64) temos que

$$
\begin{aligned}
\langle\Delta y, \eta\rangle=\sum_{i}\left\langle E_{i} E_{i}(y), \eta\right\rangle & =-\sum_{i}\left\langle E_{i}(y), E_{i}(\eta)\right\rangle \\
& =-\sum_{i}\left\langle E_{i}(y),\left(r-r_{i}\right) E_{i}(y)+E_{i}(r) y\right\rangle \\
& =-\sum_{i}\left(r-r_{i}\right)\left\langle E_{i}(y), E_{i}(y)\right\rangle \\
& =-\sum_{i}\left(r-r_{i}\right) e_{i}(x) \cdot e_{i}(x) \\
& =-\sum_{i}\left(r-r_{i}\right) r_{i}^{2} e_{i}(\xi) \cdot e_{i}(\xi) \\
& =-\sum_{i}\left(r-r_{i}\right) r_{i}^{2} d \xi\left(e_{i}\right) \cdot d \xi\left(e_{i}\right) \\
& =-\sum_{i}\left(r-r_{i}\right) r_{i}^{2} k_{i}^{2} e_{i} \cdot e_{i} \\
& =-\sum_{i}\left(r-r_{i}\right) \\
& =-2 r+\left(r_{1}+r_{2}\right) \\
& =0 .
\end{aligned}
$$

Dessa forma sabemos que $\eta \in \mathbb{V}^{\perp}$. Seja $\wp=(1,-1, \mathbf{0}, 0) \in \mathbb{R}_{2}^{6}$ o vetor definido em (2.3). Desde que $\langle y, \wp\rangle=0$, por (2.59) temos que

$$
\mathbb{V}^{\perp}=\operatorname{span}\{\eta, \wp\},\langle\eta, \eta\rangle=\langle\wp, \wp\rangle=0,\langle\eta, \wp\rangle=-1
$$

Chamamos $\eta: M \rightarrow \mathrm{C}^{5} \subset \mathbb{R}^{6}$ definido por (2.59) a aplicação de Gauss-Laguerre de $x$.

É claro que $\mathbb{V}, \mathbb{V}^{\perp}$ e $\eta$ são Laguerre invariantes: Se $x$ é Laguerre equivalente a $\tilde{x}$ por $T \in L \mathbb{G}$, então temos

$$
\tilde{\mathbb{V}}=\mathbb{V} T, \tilde{\mathbb{V}}^{\perp}=\mathbb{V}^{\perp} T, \tilde{\eta}=\eta T .
$$

Agora sejam $x, \tilde{x}: M \rightarrow \mathbb{R}^{3}$ Laguerre equivalentes por $T \in L \mathbb{G}$. Então por (2.48) e (2.66) temos que

$$
\tilde{y}=\frac{1}{b} y T, \tilde{\eta}=\eta T
$$

para uma função $b \neq 0$. Segue que

$$
\langle d \tilde{y}, d \tilde{y}\rangle=\frac{1}{b^{2}}\langle d y, d y\rangle
$$

Se $\left\{E_{1}, E_{2}\right\}$ é uma base ortonormal para $\langle d y, d y\rangle$, $\log \left\{\tilde{E}_{i}=b E_{i}\right\}$ é uma base ortonormal para $\langle d \tilde{y}, d \tilde{y}\rangle$. De (2.67) e (2.68) obtemos

$$
\sum_{i}\left\langle\tilde{E}_{i}(\tilde{\eta}), \tilde{E}_{i}(\tilde{\eta})\right\rangle=b^{2} \sum_{i}\left\langle E_{i}(\eta), E_{i}(\eta)\right\rangle
$$


E segue de (2.68) e (2.69) que

$$
\begin{aligned}
g & =\left(\sum_{i}\left\langle E_{i}(\eta), E_{i}(\eta)\right\rangle\right)\langle d y, d y\rangle \\
& =\left(\sum_{i}\left\langle E_{i}(\eta), E_{i}(\eta)\right\rangle\right) I I I
\end{aligned}
$$

é uma invariante de Laguerre. Da igualdade em (2.64) obtemos

$$
\sum_{i}\left\langle E_{i}(\eta), E_{i}(\eta)\right\rangle=\sum_{i}\left(r_{i}-r\right)^{2}
$$

Assim, sabemos que

$$
g=\left(\sum_{i}\left(r_{i}-r\right)^{2}\right) I I I
$$

é uma métrica invariante de Laguerre em qualquer ponto não umbílico de $x$. Chamamos $g$ a métrica de Laguerre de $x$. O volume de $g$ é dado por

$$
L(x)=\operatorname{Vol}_{g}(x)=\int_{M} \frac{\left(\sum_{i}\left(r_{i}-r\right)^{2}\right)}{r_{1} r_{2}} d M,
$$

onde $d M$ é a forma de volume em relação a $d x \cdot d x$.

Note que

$$
\begin{aligned}
\frac{\left(\sum_{i}\left(r_{i}-r\right)^{2}\right)}{r_{1} r_{2}} & =\frac{\left(r_{1}-r\right)^{2}+\left(r_{2}-r\right)^{2}}{r_{1} r_{2}} \\
& =\frac{\left(r_{1}-\frac{r_{1}+r_{2}}{2}\right)^{2}+\left(r_{2}-\frac{r_{1}+r_{2}}{2}\right)^{2}}{r_{1} r_{2}} \\
& =\frac{2\left(\frac{r_{1}-r_{2}}{2}\right)^{2}}{r_{1} r_{2}} \\
& =\frac{\frac{1}{2}\left(\frac{1}{k_{1}}-\frac{1}{k_{2}}\right)^{2}}{\frac{1}{k_{1} k_{2}}} \\
& =\frac{1}{2} \frac{\left(k_{1}-k_{2}\right)^{2}}{k_{1} k_{2}} \\
& =\frac{1}{2}\left(\frac{k_{1}^{2}+2 k_{1} k_{2}+k_{2}^{2}-4 k_{1} k_{2}}{k_{1} k_{2}}\right) \\
& =\frac{1}{2}\left(\frac{\left(k_{1}+k_{2}\right)^{2}-4 K}{K}\right) \\
& =2\left(\frac{H^{2}-K}{K}\right)
\end{aligned}
$$


Então equivalentemente à expressão (2.73) temos

$$
L(x)=2 \int_{M} \frac{H^{2}-K}{K} d M,
$$

que é (a menos do fator 2) o funcional de Laguerre dado no livro de Blaschke [6], os artigos de Musso e Nicolodi [15] e Palmer [18].

Definição 2.12. Chamamos às superfícies críticas do funcional de Laguerre $L(x)$ de superfícies mínimas de Laguerre.

Observação 2.4. Equivalentemente à definição anterior, em [12], uma superfície em $\mathbb{R}^{3}$ é mínima de Laguerre se, e somente se $\Delta^{I I I} r=0$. Além disso, os autores provam que $\Delta^{I I I}(x+r \xi)=0$, tal informação será útil posteriormente.

Por outra parte, suponha que $v=\alpha_{1} e_{1}+\alpha_{2} e_{2}, w=\beta_{1} e_{1}+\beta_{2} e_{2}$ são vetores tangentes à superfície, então pode-se escrever

$$
v=\frac{\alpha_{1}}{r_{1}} r_{1} e_{1}+\frac{\alpha_{2}}{r_{2}} r_{2} e_{2},
$$

assim,

$$
\begin{aligned}
v(\eta)=d \eta(v) & =\frac{\alpha_{1}}{r_{1}} E_{1}(\eta)+\frac{\alpha_{2}}{r_{2}} E_{2}(\eta) \\
& =\frac{\alpha_{1}}{r_{1}}\left[\left(r-r_{1}\right) E_{1}(y)+E_{1}(r) y\right]+\frac{\alpha_{2}}{r_{2}}\left[\left(r-r_{2}\right) E_{2}(y)+E_{2}(r) y\right] \\
& =\frac{\alpha_{1}}{r_{1}}\left(r-r_{1}\right) E_{1}(y)+\frac{\alpha_{2}}{r_{2}}\left(r-r_{2}\right) E_{2}(y)+\left[\frac{\alpha_{1}}{r_{1}} E_{1}(r)+\frac{\alpha_{2}}{r_{2}} E_{2}(r)\right] y,
\end{aligned}
$$

analogamente, obtemos

$$
w(\eta)=d \eta(w)=\frac{\beta_{1}}{r_{1}}\left(r-r_{1}\right) E_{1}(y)+\frac{\beta_{2}}{r_{2}}\left(r-r_{2}\right) E_{2}(y)+\left[\frac{\beta_{1}}{r_{1}} E_{1}(r)+\frac{\beta_{2}}{r_{2}} E_{2}(r)\right] y,
$$

logo, temos que

$$
\begin{aligned}
\langle d \eta(v), d \eta(w)\rangle & =\frac{\alpha_{1} \beta_{1}}{r_{1}^{2}}\left(r-r_{1}\right)^{2}+\frac{\alpha_{2} \beta_{2}}{r_{2}^{2}}\left(r-r_{2}\right)^{2} \\
& =\frac{\alpha_{1} \beta_{1}\left(\frac{r_{1}+r_{2}}{2}-r_{1}\right)^{2}}{r_{1}^{2}}+\frac{\alpha_{2} \beta_{2}\left(\frac{r_{1}+r_{2}}{2}-r_{2}\right)^{2}}{r_{2}^{2}} \\
& =\frac{\alpha_{1} \beta_{1}}{4 r_{1}^{2}}\left(-r_{1}+r_{2}\right)^{2}+\frac{\alpha_{2} \beta_{2}}{4 r_{2}^{2}}\left(r_{1}-r_{2}\right)^{2} \\
& =\frac{1}{4}\left(\frac{\alpha_{1} \beta_{1}}{r_{1}^{2}}+\frac{\alpha_{2} \beta_{2}}{r_{2}^{2}}\right)\left(r_{1}-r_{2}\right)^{2} .
\end{aligned}
$$

Por outro lado, como

$$
v=\frac{\alpha_{1}}{r_{1}} r_{1} e_{1}+\frac{\alpha_{2}}{r_{2}} r_{2} e_{2}
$$

temos,

$$
\begin{aligned}
& v(y)=d y(v)=\frac{\alpha_{1}}{r_{1}} E_{1}(y)+\frac{\alpha_{2}}{r_{2}} E_{2}(y), \\
& w(y)=d y(w)=\frac{\beta_{1}}{r_{1}} E_{1}(y)+\frac{\beta_{2}}{r_{2}} E_{2}(y),
\end{aligned}
$$


$\log \mathrm{O}$

$$
\langle d y(v), d y(w)\rangle=\left(\frac{\alpha_{1} \beta_{1}}{r_{1}^{2}}+\frac{\alpha_{2} \beta_{2}}{r_{2}^{2}}\right) .
$$

Finalmente, de (2.74) é fácil ver que $\frac{1}{4}\left(r_{1}-r_{2}\right)^{2}=\frac{H^{2}-K}{K^{2}}$, assim, usando (2.76) e (2.77) temos

$$
\begin{gathered}
\langle d \eta(v), d \eta(w)\rangle=\frac{1}{4}\left(r_{1}-r_{2}\right)^{2}\langle d y(v), d y(w)\rangle \\
\langle d \eta, d \eta\rangle=\left(\frac{H^{2}-K}{K^{2}}\right) I I I .
\end{gathered}
$$

Este fato, permite definir a métrica de Laguerre como $h_{L}=\left(\frac{H^{2}-K}{K^{2}}\right) I I I$ (ver [1], §2). 


\section{Teoremas de representação e} completude da métrica de Laguerre

No estudo das superícies mínimas de Laguerre tem sido apresentada uma representação holomorfa do tipo Weierstrass em termos de uma função holomorfa e duas funções meromorfas (ver [23]). No entanto, o conceito de minimalidade no sentido de Laguerre pode ser estendido para superfícies que possuem curvatura Gaussiana nula em um conjunto de pontos isolados (ver [1]), tais superfícies representam uma generalização das superícies mínimas de Laguerre. Nesta seção apresentamos uma representação global do tipo Weierstrass para tais superfícies. No que segue, abreviaremos superfície mínima de Laguerre por superfície-ML.

\subsection{Superfícies mínimas generalizadas de Laguerre}

Como já vimos até agora, uma imersão $\psi: S \rightarrow \mathbb{R}^{3}$ de uma superfície $S$ orientável e conexa em $\mathbb{R}^{3}$, com curvatura Gaussiana $K$ não nula em todos seus pontos e curvatura média $H$, é uma superfície-ML se satisfaz a equação

$$
\Delta^{\mathrm{III}}\left(\frac{H}{K}\right)=0
$$

sendo $I I I$ sua terceira forma fundamental. Motivados pela harmonicidade da função $\frac{H}{K}$, suponhamos que $\psi: S \rightarrow \mathbb{R}^{3}$ é uma superfície de modo que suas curvaturas média e Gaussiana estejam relacionadas por uma expressão do tipo

$$
H(p)=R(p) K(p), \quad \forall p \in S
$$

para alguma função diferenciável $R: S \rightarrow \mathbb{R}$. Com tais hipóteses pode-se definir a forma quadrática

$$
\widetilde{h}=I-2 R I I
$$


onde $I$ e $I I$ são, respectivamente, a primeira e a segunda forma fundamental da imersão. Esta nova forma quadrática é conforme á terceira forma fundamental da imersão se $K \neq 0$, pois de (1.10) temos

$$
I I I=-K I+2 H I I=-K \widetilde{h} .
$$

Se $K=0$ em um ponto $p \in S$ então, de $(3.2), H(p)=0$ e $I I=0$ em $p$. Em particular, $\widetilde{h}=I$ em $p$. Do anterior deduze-se que, $\widetilde{h}$ deve ser uma forma quadrática definida. Com tudo isto, já que $I I I$ é uma métrica Riemanniana sempre que $K \neq 0$ e $\widetilde{h}=I$ nos pontos onde $K=0$, então de (3.4) temos que $\widetilde{h}$ é uma métrica Riemanniana se $K \leq 0$ e também é $-\widetilde{h}$ quando $K>0$. Pode-se então enunciar o seguinte resultado.

Proposição 3.1. Seja $\psi: S \rightarrow \mathbb{R}^{3}$ uma imersão de uma superfície orientável e conexa $S$ com curvatura média $H$ e curvatura Gaussiana $K$. Se $R: S \rightarrow \mathbb{R}$ é uma função diferenciável tal que $H=R K$ então se verifica uma das seguintes afirmações:

- $K>0$ em qualquer ponto de $S$, ou

- $K \leq 0$ em qualquer ponto de $S$, e em este caso ou $K$ se anula identicamente sobre $S$ e portanto $\psi(S)$ está contida em um plano, ou $\{p \in S \mid K(p)=0\}$ é um conjunto de pontos isolados.

Demonstração. Já que a forma quadrática $\widetilde{h}=I$ dada por (3.3) está definida em qualquer ponto, então $\widetilde{h}=I$ deve ser ou definida positiva ou definida negativa em todos os pontos. Portanto, como $\widetilde{h}=I$ é definida positiva em ponto se e somente se $K \leq 0$ em esse ponto, pode-se deduzir que $K>0$ em todos os pontos ou $K \leq 0$ em todos os pontos.

Por outro lado, de (3.4), III $=-K \widetilde{h}$, que também é valido nos pontos onde $K=0$. Portanto, como $I I I:=\langle d N, d N\rangle$, onde $N$ é a aplicação de Gauss da imersão, obtemos que $N$ é uma aplicação conforme para a superfície $S$ com a estrutura induzida por $\widetilde{h}$. Assim, $d N$ se anula nos pontos isolados ou $d N$ é identicamente nula, ou equivalentemente, $K=0$ nos pontos isolados ou $\psi(S)$ é parte de um plano.

Observação 3.1. Nas condições da Proposição anterior pode-se definir a métrica Riemanniana $h$ dada por

$$
h=\left\{\begin{array}{r}
\widetilde{h}, \text { se } K \leq 0 \\
-\widetilde{h} \text {, se } K>0
\end{array}\right.
$$

Aliás a aplicação de Gauss $N$ da imersão é conforme para a métrica $h$, pois $I I I=|K| h$.

Definição 3.1. Seja $\psi: S \rightarrow \mathbb{R}^{3}$ uma imersão e $R: S \rightarrow \mathbb{R}$ uma função diferenciável tal que $H=R K$. Dizemos que $\psi$ é uma superfície minima generalizada de Laguerre, desde agora superfície-MGL, se o Laplaciano de $R$ em relação à métrica Riemanniana h é identicamente nula, isto é

$$
\Delta^{I I I} R=0 .
$$

Observe-se primeiramente que a família de imersões-ML de uma superfície $S$ são imersões-MGL de tal superfície, já que se é satisfeito que $K \neq 0$, a função média dos raios de curvatura é dada por

$$
R(p)=\frac{1}{2}\left(\frac{1}{k_{1}(p)}+\frac{1}{k_{1}(p)}\right)=\frac{H(p)}{K(p)}, \quad \forall p \in S
$$

está sempre bem definida e ela é harmônica em relação à terceira forma fundamental, que é conforme a $h$. 
No entanto, para uma superfície-MGL a curvatura Gaussiana poderia-se anular em alguns pontos. Este é um fato importante, porque por exemplo, as imersões mínimas são imersões-MGL, mas não são imersõesML em geral, pois a curvatura Gaussiana poderia-se anular em alguns pontos. Em geral, as superfícies de Weingarten que satisfazem $H=c K, c \in \mathbb{R}$, são superfícies-MGL.

Note que os exemplos 2.1 e 2.2 tratam, respectivamente, de isometrias de $\mathbb{R}^{3}$ e de deslocamento paralelo ao longo da normal. Assim, podemos obter superfícies-MGL pelos seguintes dois exemplos.

Exemplo 3.1 (Isometrias). Dada $\psi: S \rightarrow \mathbb{R}$ uma imersão de uma superfície-MGL $S$ e uma isometria $\phi: \mathbb{R}^{3} \rightarrow \mathbb{R}^{3}$, então $\widetilde{\psi}=\psi \circ \phi$ é também uma imersão de uma superfície-MGL, verificando-se

$$
\widetilde{H}=H, \quad \widetilde{K}=K, \quad \widetilde{R}=R .
$$

Exemplo 3.2 (Superfícies Paralelas). Seja $\psi: S \rightarrow \mathbb{R}^{3}$ uma superfície-MGL com aplicação de Gauss $N$, curvatura Gaussiana $K$ e curvatura média $H$. Então a superfície paralela a $S$ com distância a $\in \mathbb{R}$, $\widetilde{\psi}=\psi+a N$, sempre que $1-2 a H+a^{2} K \neq 0$, tem curvatura Gaussiana e curvatura média dadas, respectivamente, por

$$
\begin{aligned}
\widetilde{K} & =\frac{K}{1-2 a H+a^{2} K}, \\
\widetilde{H} & =\frac{H-a K}{1-2 a H+a^{2} K} .
\end{aligned}
$$

Como as aplicações de Gauss de ambas imersões coincidem, então a terceira forma fundamental também coincide. Aliás $\widetilde{R}=\frac{\widetilde{H}}{\widetilde{K}}=\frac{H-a K}{K}=\frac{H}{K}-a=R-a$, logo $\Delta^{I I I} \widetilde{R}=\Delta^{I I I}(R-a)=\Delta^{I I I} R=0$, e portanto temos que $\widetilde{\psi}$ é também uma superfície-MGL.

Além disso, podem-se gerar superfícies-MGL a partir de superfícies homotéticas.

Exemplo 3.3 (Superfícies homotéticas). Se $\psi: S \rightarrow \mathbb{R}^{3}$ é uma imersão de uma superfície-MGL, então $\widetilde{\psi}=c \psi$ para algum $c \in \mathbb{R} \backslash\{0\}$ é também a imersão de uma superfície-MGL com $\widetilde{R}=c R$. É suficiente observar que $\widetilde{K}=\frac{K}{c^{2}}, \widetilde{H}=\frac{H}{c}$ e que a terceira forma fundamental de ambas imersões coincidem.

\subsection{Representação conforme para superfícies-MGL}

Em esta seção obteremos uma representação conforme global explicita para superfícies-MGL, que dependera de três dados geométricos: uma função real harmônica, uma função meromorfa e uma 1-forma holomorfa.

Seja $S^{2}$ a esfera unitária em $\mathbb{R}^{3}$ e considere as aplicações

$$
\begin{gathered}
\pi: S^{2} \rightarrow \mathbb{C} \cup\{\infty\} \\
\left(x_{1}, x_{2}, x_{3}\right) \mapsto \pi\left(x_{1}, x_{2}, x_{3}\right)=\left\{\begin{array}{cc}
\frac{x_{1}+i x_{2}}{1-x_{3}}, \quad\left(x_{1}, x_{2}, x_{3}\right) \neq(0,0,1) \\
\infty, \quad \text { c.c. }
\end{array}\right. \\
\pi^{-1}: \mathbb{C} \cup\{\infty\} \rightarrow S^{2} \\
z \mapsto \pi^{-1}(z)=\left\{\begin{array}{cc}
\left(\frac{2 \operatorname{Re}(z)}{1+|z|^{2}}, \frac{2 \operatorname{Im}(z)}{1+|z|^{2}}, \frac{|z|^{2}-1}{1+|z|^{2}}\right), & z \neq \infty \\
(0,0,1) \quad, \quad z=\infty .
\end{array}\right.
\end{gathered}
$$

Tais aplicações são bijetivas, conformes e invertem a orientação, chamamos a aplicação $\pi$ de projeção estereográfica. 
Se $\psi: S \rightarrow \mathbb{R}^{3}$ é uma imersão de uma superfície orientável e conexa $S$ em $\mathbb{R}^{3}, N$ sua aplicação de Gauss e $\pi$ a projeção estereográfica, então a aplicação $g$ definida por $g=\pi \circ N$, é chamada também aplicação de Gauss da imersão. Mais precisamente, se o vetor normal em cada ponto $p$ da superfície é $N(p)=\left(N_{1}(p), N_{2}(p), N_{3}(p)\right)$ então

$$
g(p)=\frac{N_{1}(p)+i N_{2}(p)}{1-N_{3}(p)}
$$

Reciprocamente, também teremos que

$$
N=\left(\frac{g+\bar{g}}{1+|g|^{2}},-i \frac{g-\bar{g}}{1+|g|^{2}}, \frac{|g|^{2}-1}{1+|g|^{2}}\right) .
$$

Lembremos também o seguinte resultado.

Lema 3.1 (Lema de Representação). Seja $\psi: S \rightarrow \mathbb{R}^{3}$ uma imersão de uma superfície $S$ simplesmente conexa, sendo $\left(\psi_{1}, \psi_{2}, \psi_{3}\right)$ suas funções componentes, e consideremos as funções $\left(\phi_{1}, \phi_{2}, \phi_{3}\right)$ onde

$$
\phi_{k}=\frac{\partial \psi_{k}}{\partial z}, \forall k \in\{1,2,3\}
$$

então verifica-se que

$$
\psi_{k}=2 \operatorname{Re}\left\{\int \phi_{k} d z\right\}+c_{k}, \forall k \in\{1,2,3\}
$$

para algumas constantes $c_{k} \in \mathbb{C}$.

Demonstração. Suponha que $z=u+i v$, então

$$
\begin{aligned}
\phi_{k} d z & =\frac{\partial \psi_{k}}{\partial z} d z=\frac{1}{2}\left(\frac{\partial}{\partial u} \psi_{k}-i \frac{\partial}{\partial v} \psi_{k}\right)(d u+i d v) \\
& =\frac{1}{2}\left[\left(\frac{\partial \psi_{k}}{\partial u} d u+\frac{\partial \psi_{k}}{\partial v} d v\right)+i\left(\frac{\partial \psi_{k}}{\partial u} d v-\frac{\partial \psi_{k}}{\partial v} d u\right)\right] \\
\bar{\phi}_{k} \overline{d z} & =\overline{\left(\frac{\partial \psi_{k}}{\partial z}\right)} \overline{(d u+i d v)}=\frac{1}{2}\left(\frac{\partial \psi_{k}}{\partial u}+i \frac{\partial \psi_{k}}{\partial v}\right)(d u-i d v) \\
& =\frac{1}{2}\left[\left(\frac{\partial \psi_{k}}{\partial u} d u+\frac{\partial \psi_{k}}{\partial v} d v\right)+i\left(\frac{\partial \psi_{k}}{\partial v} d u-\frac{\partial \psi_{k}}{\partial u} d v\right)\right]
\end{aligned}
$$

onde concluímos

$$
\overline{\phi_{k} d z}=\overline{\phi_{k}} d z \text {. }
$$

Agora, já que $\psi$ só tem parte real

$$
\frac{\partial \psi_{k}}{\partial \bar{z}}=\overline{\left(\frac{\partial \overline{\psi_{k}}}{\partial z}\right)}=\overline{\left(\frac{\partial \psi_{k}}{\partial z}\right)}=\overline{\phi_{k}}
$$

Portanto,

$$
\begin{aligned}
d \psi_{k} & =\frac{\partial \psi_{k}}{\partial z} d z+\frac{\partial \psi_{k}}{\partial \bar{z}} d \bar{z}=\phi_{k} d z+\overline{\phi_{k}} \overline{d z} \\
& =\phi_{k} d z+\overline{\phi_{k} d z}=2 \operatorname{Re}\left\{\phi_{k} d z\right\}
\end{aligned}
$$


de onde obtemos, para algumas constantes $c_{k} \in \mathbb{C}, k \in\{1,2,3\}$ que:

$$
\psi_{k}=2 \operatorname{Re} \int \phi_{k} d z+c_{k}
$$

No que segue, se $\psi: S \rightarrow \mathbb{R}^{3}$ é uma superfície-MGL, consideraremos $S$ como uma superfície de Riemann com a estrutura conforme induzida pela métrica $h$. Portanto, da Observação $3.1, N$ será conforme para a métrica $h$, ou equivalentemente, $g: S \rightarrow \mathbb{C} \cup\{\infty\}$ será uma função meromorfa. Por outra parte, já que $\Delta^{h} R=0$, temos que $R$ é uma função real harmônica na superfície $S$. Aliás, para quaisquer parâmetro conforme local $z$ da superfície de Riemann $S$, supondo que $\lambda=\left|\psi_{u}\right|=\left|\psi_{v}\right|$, temos

$$
\frac{\partial}{\partial \bar{z}} \frac{\partial}{\partial z} R=\frac{4}{\lambda^{2}} \Delta^{h} R=0
$$

$\operatorname{logo} \frac{\partial}{\partial z} R$ é uma 1 -forma holomorfa denotada por $\partial R$, sendo também conhecida como a parte $(1,0)$ de $d R$.

Teorema 3.1. Seja $\psi: S \rightarrow \mathbb{R}^{3}$ uma superfície-MGL não plana, com aplicação de Gauss g, e tal que $H=R K$. Então existe uma 1 -forma holomorfa $w$ tal que a imersão $\psi$ pode-se recuperar como

$$
\psi=2 \operatorname{Re}\left\{\int\left(\phi_{1}, \phi_{2}, \phi_{3}\right)\right\}-R N
$$

onde

$$
\left\{\begin{array}{l}
\phi_{1}=\frac{1}{2}\left(\frac{\partial R+w}{g}+g(\partial R-w)\right) \\
\phi_{2}=\frac{i}{2}\left(\frac{\partial R+w}{g}-g(\partial R-w)\right) \\
\phi_{3}=w
\end{array}\right.
$$

Reciprocamente, seja $S$ uma superficie de Riemann simplesmente conexa, $g: S \rightarrow \mathbb{C} \cup\{\infty\}$ uma função meromorfa não constante, w uma 1 -forma holomorfa e $R: S \rightarrow \mathbb{R}$ uma função real harmônica, tal que se g tem um zero (respectivamente, um polo) de ordem $n \in \mathbb{N}$ em $p \in S$, então $\partial R+w$ (respectivamente, $\partial R-w)$ tem um zero de ordem maior o igual que n em p. Então (3.7) define uma superfície-MGL sempre que

$$
\left|\frac{\partial R+w}{g}+\bar{g}(w-\partial R)\right|^{2}-R^{2} \frac{4|d g|^{2}}{\left(1+|g|^{2}\right)^{2}} \neq 0
$$

com aplicação de Gauss g e $H=R K$.

Demonstração. Já que $\psi$ é uma imersão não plana, então sabe-se pela Proposição 3.1 que os pontos onde a curvatura de Gauss se anula são isolados. Assim, se denotamos por $S_{0}=\{p \in S \mid K(p) \neq 0\}$, então $\psi: S_{0} \rightarrow \mathbb{R}^{3}$ é uma superfície-ML e da Observação 2.4 temos

$$
\Delta^{\mathrm{III}}(\psi+R N)=0, \forall p \in S_{0}
$$

Aliás, com $h$ e III são métricas Riemannianas conformes sobre $S_{0}$, então $\Delta^{h}(\psi+R N)=0$ sobre $S_{0}$ e portanto também sobre $S$ pela continuidade. Assim, podem-se considerar as três 1-formas holomorfas

$$
\Phi_{k}=\partial\left(\psi_{k}+R N_{k}\right), k=1,2,3 .
$$


Se $z$ é um parâmetro conforme local para $h$, então a terceira e a segunda forma fundamental da imersão podem-se expressar em termos de $d z, d \bar{z}$. Para isso, usamos o fato de $N$ ter parâmetros $(u, v)$ conformes, assim, $\left|N_{u}\right|=\left|N_{v}\right|$ e $\left\langle N_{u}, N_{v}\right\rangle=0$, daí temos

$$
\begin{aligned}
\left\langle N_{z}, N_{z}\right\rangle & =\frac{1}{4}\left\langle N_{u}-i N_{v}, N_{u}-i N_{v}\right\rangle \\
& \frac{1}{4}\left\{\left|N_{u}\right|^{2}-2 i\left\langle N_{u}, N_{v}\right\rangle-\left|N_{u}\right|^{2}\right\rangle \\
& =0
\end{aligned}
$$

e

$$
\begin{aligned}
\left\langle N_{\bar{z}}, N_{\bar{z}}\right\rangle & =\frac{1}{4}\left\langle N_{u}+i N_{v}, N_{u}+i N_{v}\right\rangle \\
& \frac{1}{4}\left\{\left|N_{u}\right|^{2}+2 i\left\langle N_{u}, N_{v}\right\rangle-\left|N_{v}\right|^{2}\right\} \\
& =0
\end{aligned}
$$

$\log 0$

$$
\begin{aligned}
I I I & =\langle d N, d N\rangle \\
& =\left\langle N_{z} d z+N_{\bar{z}} d \bar{z}, N_{z} d z+N_{\bar{z}} d \bar{z}\right\rangle \\
& =\left\langle N_{z}, N_{z}\right\rangle d z^{2}+2\left\langle N_{z}, N_{\bar{z}}\right\rangle|d z|^{2}+\left\langle N_{\bar{z}}, N_{\bar{z}}\right\rangle d \bar{z}^{2} \\
& =2\left\langle N_{z}, N_{\bar{z}}\right\rangle|d z|^{2} .
\end{aligned}
$$

Por outra parte, como $d z=d u+i d v$, temos

$$
\begin{aligned}
& d u=\frac{1}{2}(d z+d \bar{z}), \\
& d v=\frac{i}{2}(d \bar{z}-d z)
\end{aligned}
$$

consequentemente, temos

$$
\begin{aligned}
d u^{2} & =\frac{1}{4}\left(d z^{2}+2|d z|^{2}+d \bar{z}^{2}\right), \\
d v^{2} & =\frac{1}{4}\left(-d z^{2}+2|d z|^{2}-d \bar{z}^{2}\right), \\
d u d v & =\frac{i}{4}\left(d \bar{z}^{2}-d z^{2}\right),
\end{aligned}
$$

substituindo estas três ultimas igualdades na segunda forma fundamental na equação (1.8), temos

$$
\begin{aligned}
I I & =e d u^{2}+2 f d u d v+g d v^{2} \\
& =\frac{e}{4}\left(d z^{2}+2|d z|^{2}+d \bar{z}^{2}\right)+\frac{f}{2}\left(d \bar{z}^{2}-d z^{2}\right) i+\frac{g}{4}\left(-d z^{2}+2|d z|^{2}-d \bar{z}^{2}\right) \\
& =\frac{1}{4}(e-2 f i-g) d z^{2}+\frac{1}{2}(e+g)|d z|^{2}+\frac{1}{4}(e-2 f i-g) d \bar{z}^{2},
\end{aligned}
$$


logo, calculando os coeficientes da segunda forma fundamental nos novos parâmetros complexos temos

$$
\begin{aligned}
e-2 f i-g & =e-f i-f i-g \\
& =-\left\{\left\langle\psi_{u}, N_{u}\right\rangle-\left\langle\psi_{u}, N_{v}\right\rangle i-\left\langle\psi_{v}, N_{u}\right\rangle i-\left\langle\psi_{v}, N_{v}\right\rangle\right\} \\
& =-\left\{\left\langle\psi_{u}-i \psi_{v}, N_{u}-i N_{v}\right\rangle\right\} \\
& =4\left\langle\psi_{z},-N_{z}\right\rangle . \\
e+g & =e-f i+f i+g \\
& =-\left\{\left\langle\psi_{u}, N_{u}\right\rangle+\left\langle\psi_{u}, N_{v}\right\rangle i-\left\langle\psi_{v}, N_{u}\right\rangle i+\left\langle\psi_{v}, N_{v}\right\rangle\right\} \\
& =-\left\{\left\langle\psi_{u}-i \psi_{v}, N_{u}+i N_{v}\right\rangle\right\} \\
& =4\left\langle\psi_{z},-N_{\bar{z}}\right\rangle . \\
e+2 i-g & =e+f i+f i-g \\
& =-\left\{\left\langle\psi_{u}, N_{u}\right\rangle+\left\langle\psi_{u}, N_{v}\right\rangle i+\left\langle\psi_{v}, N_{u}\right\rangle i-\left\langle\psi_{v}, N_{v}\right\rangle\right\} \\
& =-\left\{\left\langle\psi_{u}+i \psi_{v}, N_{u}+i N_{v}\right\rangle\right\} \\
& =4\left\langle\psi_{\bar{z}},-N_{\bar{z}}\right\rangle .
\end{aligned}
$$

Finalmente, substituindo as equações (3.12), (3.13) e (3.14) na última expressão da segunda forma fundamental temos

$$
I I=\left\langle\psi_{z},-N_{z}\right\rangle d z^{2}+2\left\langle\psi_{z},-N_{\bar{z}}\right\rangle|d z|^{2}+\left\langle\psi_{\bar{z}},-N_{\bar{z}}\right\rangle d \bar{z}^{2}
$$

Logo, de (1.11) temos

$$
\begin{aligned}
& I I I=2 \underbrace{\left\langle N_{z}, N_{\bar{z}}\right\rangle}_{\tilde{M}^{\prime}}|d z|^{2}, \\
& I I=\underbrace{\left\langle\psi_{z},-N_{z}\right\rangle}_{L^{\prime}} d z^{2}+2 \underbrace{\left\langle\psi_{z},-N_{\bar{z}}\right\rangle}_{M^{\prime}}|d z|^{2}+\underbrace{\left\langle\psi_{\bar{z}},-N_{\bar{z}}\right\rangle}_{N^{\prime}} d \bar{z}^{2},
\end{aligned}
$$

então

$$
H(I I I, I I)=\frac{-2 \tilde{M}^{\prime} M^{\prime}}{-2 \tilde{M}^{\prime 2}}=\frac{M^{\prime}}{\tilde{M}^{\prime}}=\frac{\left\langle\phi_{z},-N_{\bar{z}}\right\rangle}{\left\langle N_{z}, N_{\bar{z}}\right\rangle}=\frac{H}{K}=R,
$$

sobre $S_{0}$ e portanto, pela continuidade, $\left\langle\psi_{z}+R N_{z}, N_{\bar{z}}\right\rangle=0$ sobre $S$. Daí, obtemos

$$
\left\langle\left(\phi_{1}, \phi_{2}, \phi_{3}\right), N_{\bar{z}}\right\rangle=\left\langle(\psi+R N)_{z}, N_{\bar{z}}\right\rangle=\left\langle\psi_{z}+R N_{z}, N_{\bar{z}}\right\rangle=0
$$

onde $\Phi_{k}=\phi_{k} d z, \forall k=1,2,3$. Aliás, da definição de $N$ em (3.5), temos

$$
N_{\bar{z}}=\frac{\overline{g_{z}}}{\left(1+|g|^{2}\right)^{2}}\left(1-g^{2}, i\left(1+g^{2}\right), 2 g\right)
$$

e de (3.16) obtemos

$$
\left(1-g^{2}\right) \phi_{1}+i\left(1+g^{2}\right) \phi_{2}+2 g \phi_{3}=0
$$

Por outro lado, obtemos que $R_{z}=\left\langle(\psi+R N)_{z}, N\right\rangle=\left\langle\left(\phi_{1}, \phi_{2}, \phi_{3}\right), N\right\rangle$, isto é, usando (3.5)

$$
(g-\bar{g}) \phi-i(g-\bar{g}) \phi_{2}+\left(|g|^{2}-1\right) \phi_{3}=R_{z}\left(1+|g|^{2}\right)
$$


Fazendo uso de (3.17) e (3.18), as funções $\phi_{1}$ e $\phi_{2}$ podem ser obtidas como

$$
\begin{aligned}
2 \phi_{1} & =\frac{R_{z}+\phi_{3}}{g}+g\left(R_{z}-\phi_{3}\right), \\
2 \phi_{2} & =i \frac{R_{z}+\phi_{3}}{g}-i g\left(R_{z}-\phi_{3}\right) .
\end{aligned}
$$

Portanto, tomando $w=\Phi_{3}$ e usando o Lema (3.1), obtemos a partir de

$$
\phi_{z}+(R N)_{z}=\left(\phi_{1}, \phi_{2}, \phi_{3}\right)
$$

a fórmula de representação (3.7). Reciprocamente, seja $S$ uma superfície Riemanniana e considere a aplicação $\psi: S \rightarrow \mathbb{R}^{3}$ dada por (3.7). A partir das condições entre os zeros e polos de $g, \partial R+w$ e $\partial R-w$, obtemos que as três 1 -formas que se integram a expressão (3.7) estão bem definidas sobre $S$ e portanto também estará a aplicação $\psi$. Seja $z$ um parâmetro local conforme para $S$ e tomemos $w=\phi_{3} d z$, então

$$
\left\langle\psi_{z}, \psi_{\bar{z}}\right\rangle^{2}-\left\langle\psi_{z}, \psi_{z}\right\rangle\left\langle\psi_{\bar{z}}, \psi_{\bar{z}}\right\rangle=\left(\frac{\mu_{1}^{4}\left|\phi_{3}\right|^{2}-4|g|\left|g_{z}\right|^{2} R^{2}+\mu_{1}^{2} \mu_{2}^{2}\left|R^{z}\right|^{2}+\mu_{1}^{3} \mu_{1}\left(\phi_{3} R_{\bar{z}}+\bar{\phi}_{3} R_{z}\right)}{2|g|^{2} \mu_{1}^{2}}\right)^{2}
$$

onde $\mu_{1}=1+|g|^{2}$ e $\mu_{2}=1-|g|^{2}$. Assim, $\psi$ é uma imersão se e só se satisfaz (3.9). Como $\left\langle\psi_{z}, N\right\rangle=$ $\frac{1}{2}\left\langle\psi_{u}, N\right\rangle-\frac{i}{2}\left\langle\psi_{v}, N\right\rangle=0$, temos que $N$, ou equivalentemente $g$, é a aplicação de Gauss da imersão. Aliás, a segunda e terceira forma fundamental estão determinadas por

$$
\begin{aligned}
I I & =-\left(w+\frac{1-|g|^{2}}{1+|g|^{2}} \partial R\right) \frac{d g}{g}+\frac{4 R|d g|^{2}}{\left(1+|g|^{2}\right)^{2}}-\left(\bar{w}+\frac{1-|g|^{2}}{1+|g|^{2}} \bar{\partial} R\right) \frac{d \bar{g}}{\bar{g}}, \\
I I I & =\frac{4|d g|^{2}}{\left(1+|g|^{2}\right)^{2}} .
\end{aligned}
$$

Assim, em qualquer ponto onde $K \neq 0$, ou equivalentemente quando $g_{z} \neq 0$, temos por $(3.15)$ que

$$
\frac{H}{K}=\frac{\left\langle\psi_{z},-N_{\bar{z}}\right\rangle}{\left\langle N_{z}, N_{\bar{z}}\right\rangle}=R
$$

e portanto $H=R K$ sobre $S$ pela continuidade. Aliás, já que a terceira forma fundamental e a métrica Reimeanniana $h$ são conformes, e $\left\langle N_{z}, N_{z}\right\rangle \equiv 0$, a estrutura conforme dada por $L$ e a de $S$. Portanto, $\Delta^{h} R=0$, isto é, $\psi$ é uma superfície-MGL.

Esta representação conforme coincide quando $R=0$ com a representação clássica de Weierstrass para superfícies mínimas para o par de dados geométricos $\left(g, \phi_{3}\right)$, obtendo-se

$$
\psi=2 \operatorname{Re}\left\{\int\left(\phi_{3} \frac{1-g^{2}}{2 g}, i \phi_{3} \frac{1+g^{2}}{2 g}, \phi_{3}\right) d z\right\} .
$$

Com o que pode-se obter todas a superfícies mínimas clássicas com os dados de Weierstrass já conhecidos. Também é válida para as superfícies lineares de Weingarten. Satisfazendo $H=c_{0} K, c_{0} \in \mathbb{R}$. Aliás, se $\psi$ é a parte de um plano, a representação prévia também será satisfeita tomando $g$ como uma constante. 
Finalmente, de de (1.12) temos

$$
\begin{aligned}
I I & =\underbrace{-\left(w+\frac{1-|g|^{2}}{1+|g|^{2}} \partial R\right) \frac{g_{z}}{g}}_{L^{\prime \prime}} d z+2 \underbrace{\frac{2 R\left|g_{z}\right|^{2}}{\left(1+|g|^{2}\right)^{2}}}_{M^{\prime \prime}}|d z|^{2}+\underbrace{-\left(\bar{w}+\frac{1-|g|^{2}}{1+|g|^{2}} \bar{\partial}\right) \frac{\bar{g}_{\bar{z}}}{\bar{g}}}_{N^{\prime \prime}} d \bar{z} \\
I I I & =2 \underbrace{\frac{2\left|g_{z}\right|^{2}}{\left(1+|g|^{2}\right)^{2}}}_{\tilde{M}^{\prime \prime}}|d z|^{2} .
\end{aligned}
$$

Note que $L^{\prime \prime}=-\left(w+\frac{1-|g|^{2}}{1+|g|^{2}} \partial R\right) \frac{g_{z}}{g}=-\left(\frac{(w+\partial R)}{g}+\bar{g}(w-\partial R)\right) \frac{g_{z}}{\left(1+|g|^{2}\right)}=\overline{N^{\prime \prime}}$.

Logo, de (1.12) temos

$$
\begin{aligned}
K & =\frac{\frac{4\left|g_{z}\right|^{4}}{\left(1+|g|^{2}\right)^{4}}}{\frac{4 R^{2}\left|g_{z}\right|^{4}}{\left(1+|g|^{2}\right)^{4}}-\frac{\left|g_{z}\right|^{2}}{\left(1+|g|^{2}\right)^{2}}-\left|\frac{(w+\partial R)}{g}+\bar{g}(w-\partial R)\right|^{2} \frac{1}{|d z|^{2}}} \\
& =\frac{4}{4 R^{2}-\left(1+|g|^{2}\right)^{2}\left|\frac{(w+\partial R)}{g}+\bar{g}(w-\partial R)\right|^{2} \frac{1}{\left|g_{z}\right|^{2}|d z|^{2}}}
\end{aligned}
$$

e como $d g=g_{z} d z$, temos que

$$
K=\frac{4|d g|^{2}}{4 R^{2}|d g|^{2}-\left(1+|g|^{2}\right)^{2}\left|\frac{w+\partial R}{g}+\bar{g}(w-\partial R)\right|^{2}}
$$

Pode-se obter de forma análoga um teorema de representação utilizando como dados a aplicação de Gauss $g$ e as 1-formas holomorfas $\phi_{1}$ e $\phi_{2}$. Em este caso é obtido o seguinte resultado:

Teorema 3.2. Seja $\psi: S \rightarrow \mathbb{R}^{3}$ uma imersão de uma superfície-MGL, $S$ orientável e simplesmente conexa com aplicação de Gauss g. Então, se z é um parâmetro conforme para h, existem dois 1-formas $\phi_{1}$ e $\phi_{2}$ holomorfas que junto com a aplicação de Gauss g permitem recuperar a imersão $\psi$ mediante

$$
\psi=2 \operatorname{Re}\left\{\int\left(\phi_{1}, \phi_{2}, \frac{g^{2}-1}{2 g} \phi_{1}-i \frac{g^{2}+1}{2 g} \phi_{2}\right) d z\right\}-R N
$$

onde $N$ está determinada por (3.5) e $R$ é uma função real harmônica para $h$ que pode ser escrita como

$$
R=\operatorname{Re}\left\{\int\left(\frac{\phi_{1}+i \phi_{2}}{g}+\left(\phi_{1}-i \phi_{2}\right) g\right) d z\right\}
$$

Exemplo 3.4. Seja $g(z)=\frac{2}{z}, R(z)=z+\bar{z}, \phi_{3}=z+1$. 
- Calculamos $\phi_{1}$,

$$
\begin{aligned}
2 \phi_{1} & =\frac{R_{z}+\phi_{3}}{g}+g\left(R_{z}-\phi_{3}\right), \text { como } R_{z}=1 \text { e } \phi_{3}=z+1 \\
2 \phi_{1} & =z \frac{[1+(z+1)]}{2}+\frac{2}{z}(1-(z+1)) \\
& =\frac{z}{2}(z+2)-\frac{2}{z}(z)
\end{aligned}
$$

então,

$$
\phi_{1}=\frac{z^{2}}{4}+\frac{z}{2}-1
$$

- Calculamos $\phi_{2}$,

$$
\begin{aligned}
2 \phi_{2} & =i\left(\frac{R_{z}+\phi_{3}}{g}\right)-i g\left(R_{z}-\phi_{3}\right) \\
& =i\left(z \frac{[1+(z+1)]}{2}\right)-i \frac{2}{z}(1-(z+1)) \\
& =\frac{i}{2} z(z+2)+i \frac{2}{z}(z) \\
& =\frac{i}{2}\left(z^{2}+2 z\right)+2 i
\end{aligned}
$$

então

$$
\phi_{2}=i\left(\frac{z^{2}}{4}+\frac{z}{2}\right)+i
$$

- Já conhecemos $\phi_{3}$,

$$
\phi_{3}=z+1
$$

- Calculamos $N$

$$
\begin{aligned}
N & =\left(\frac{g+\bar{g}}{1+|g|^{2}},-i\left(\frac{g-\bar{g}}{1+|g|^{2}}\right), \frac{-1+|g|^{2}}{1+|g|^{2}}\right) \\
& =\frac{1}{1+\left|\frac{2}{z}\right|^{2}}\left(\frac{2}{z}+\frac{2}{\bar{z}},-i\left(\frac{2}{z}-\frac{2}{\bar{z}}\right),-1+\left|\frac{2}{z}\right|^{2}\right) \\
& =\frac{|z|^{2}}{|z|^{2}+4}\left(\frac{2(z+\bar{z})}{z \cdot \bar{z}},-i \frac{2(\bar{z}-z)}{z \cdot \bar{z}}, \frac{-|z|^{2}+4}{|z|^{2}}\right)
\end{aligned}
$$

assim,

$$
N=\left(\frac{2(\bar{z}+z)}{|z|^{2}+4},-\frac{2 i(\bar{z}-z)}{|z|^{2}+4}, \frac{-|z|^{2}+4}{|z|^{2}+4}\right)
$$

No seguinte cálculo usaremos o fato de ser $R=z+\bar{z}=2 u$.

- Calculando NR.

$$
\begin{aligned}
N R & =\left(\frac{8 u^{2}}{|z|^{2}+4}, \frac{-8 u v}{|z|^{2}+4},\left\{\frac{-|z|^{2}+4}{|z|^{2}+4}\right\} 2 u\right) \\
& =\frac{1}{u^{2}+v^{2}+4}\left(8 u^{2},-8 u v, 2 u\left(-\left(u^{2}+v^{2}\right)+4\right)\right) \\
& =\frac{1}{u^{2}+v^{2}+4}\left(8 u^{2},-8 u v,-2 u^{3}-2 u v^{2}+8 u\right)
\end{aligned}
$$


$\log$,

$$
\begin{aligned}
\phi(u, v)= & 2 \operatorname{Re} \int\left(\frac{z^{2}}{4}+\frac{z}{2}-1, i\left(\frac{z^{2}}{4}+\frac{z}{2}+1\right), z+1\right) d z-\frac{1}{u^{2}+v^{2}+4}\left(4 u,-8 u v,-2 u^{3}-2 u v^{2}+8 u\right) \\
= & 2 \operatorname{Re}\left(\frac{z^{3}}{12}+\frac{z^{2}}{4}-z, i\left(\frac{z^{3}}{12}+\frac{z^{2}}{4}+z\right), \frac{z^{2}}{z}+z\right)-\frac{1}{u^{2}+v^{2}+4}\left(8 u^{2},-8 u v,-2 u^{3}-2 u v^{2}+8 u\right) \\
= & 2 \operatorname{Re}\left[\left(\frac{u^{3}+3 u^{2}(i v)+3 u(i v)^{2}+(i v)^{3}}{12}+\frac{u^{2}+2 u v i-v^{2}-(u+i v)}{4}\right),\right. \\
& i\left(\frac{u^{3}+3 u^{2}(i v)-3 u v^{2}-i v^{3}}{12}+\frac{u^{2}+2 u v i-v^{2}}{2}+(u+i v)\right), \\
& \left.\quad \frac{u^{2}+2 u v i-v^{2}+2(u+i v)}{2}\right]-\frac{1}{u^{2}+v^{2}+4}\left(8 u^{2},-8 u v,-2 u^{3}-2 u v^{2}+8 u\right) \\
= & 2\left(\frac{u^{3}-3 u v^{2}}{12}+\frac{u^{2}-v^{2}}{4}-u, \frac{-3 u^{2} v+v^{3}}{12}-\frac{2 u v}{4}-v, \frac{u^{2}-v^{2}+2 u}{2}\right) \\
& -\frac{1}{u^{2}+v^{2}+4}\left(8 u^{2},-8 u v,-2 u^{3}-2 u v^{2}+8 u\right) .
\end{aligned}
$$

Assim, supondo $\phi(u, v)=(x(u, v), y(u, v), z(u, v))$, temos

$$
\begin{aligned}
& x(u, v)=\frac{1}{6}\left(u^{3}-3 u v^{2}+3 u^{2}-3 v^{2}-12 u\right)-\frac{8 u^{2}}{4+u^{2}+v^{2}} \\
& y(u, v)=\frac{1}{6}\left(-3 u^{2} v+v^{3}-6 u v-12 v\right)+\frac{8 u v}{4+u^{2}+v^{2}} \\
& z(u, v)=u^{2}-v^{2}+2 u\left(1-\frac{4-u^{2}-v^{2}}{4+u^{2}+v^{2}}\right)
\end{aligned}
$$

Portanto, temos a superfície-ML $S_{1}:=\phi\left(\mathbb{R}^{2}\right)$ dada na figura a seguir.
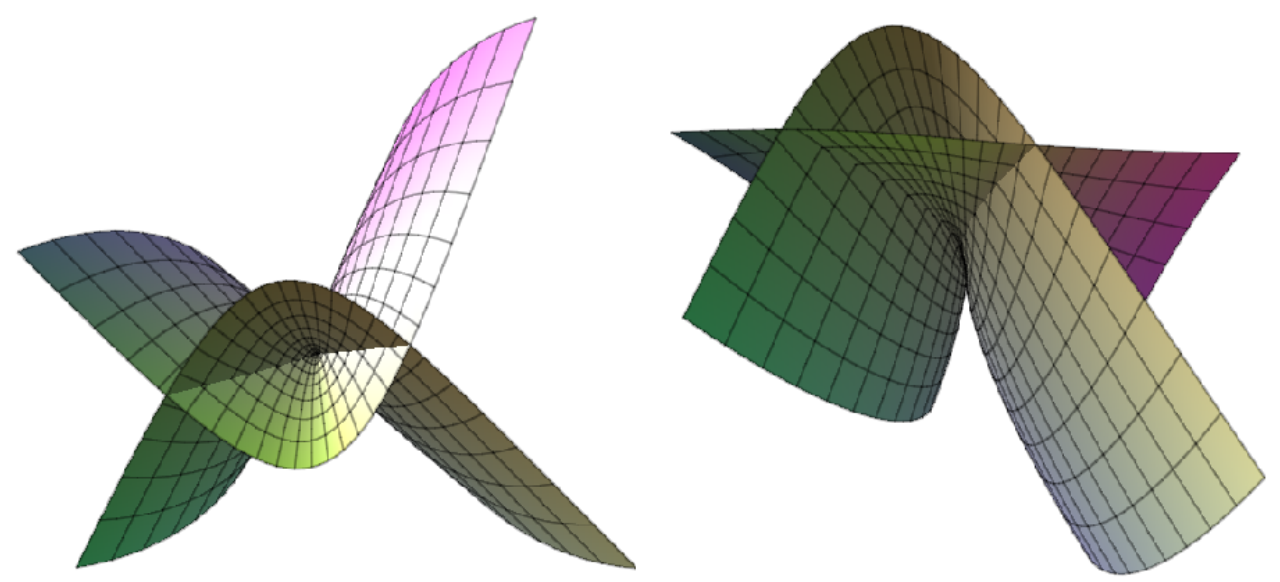

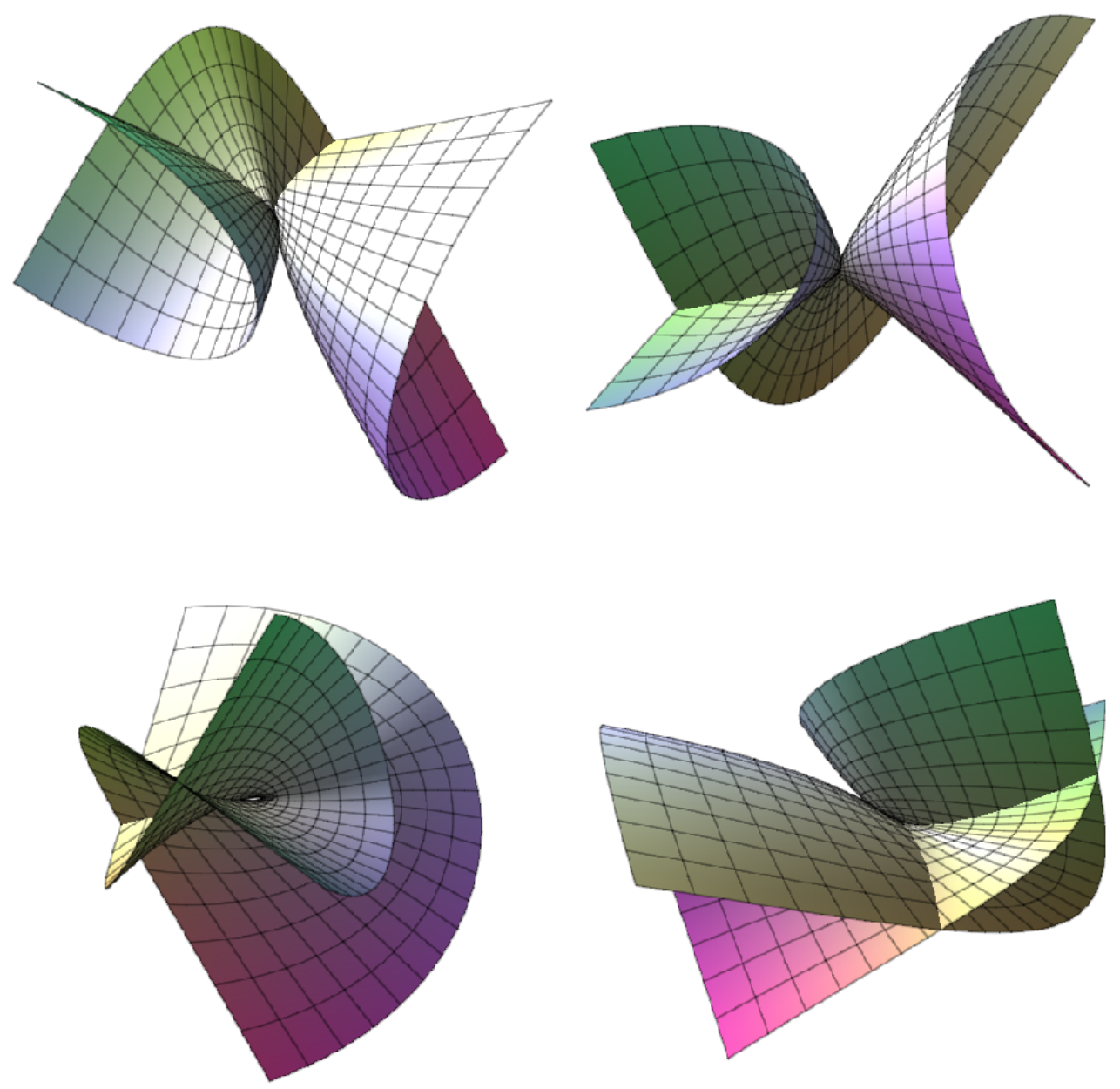

Exemplo 3.5 (Superfície de Weingarten). Seja $g(z)=z, R(z)=1, \varphi_{3}=z$.

- Calculamos $\varphi_{1}$,

$$
\begin{aligned}
& 2 \varphi_{1}=\frac{R_{z}+\varphi_{3}}{g}+g\left(R_{z}-\varphi_{3}\right), \text { como } R_{z}=0 \text { e } \varphi_{3}=z \\
& 2 \varphi_{1}=\frac{z}{z}+z(-z)=1-z^{2}
\end{aligned}
$$

então,

$$
\varphi_{1}=\frac{1}{2}-\frac{z^{2}}{2}=\frac{1}{2}\left(1-z^{2}\right) .
$$

- Calculamos $\varphi_{2}$,

$$
\begin{aligned}
2 \varphi_{2} & =i\left(\frac{R_{z}+\varphi_{3}}{g}\right)-i g\left(R_{z}-\varphi_{3}\right) \\
& =i\left(\frac{z}{z}\right)-i z(-z)=i+i z^{2}
\end{aligned}
$$

então

$$
\varphi_{2}=\frac{i}{2}\left(1+z^{2}\right) .
$$

- Já conhecemos $\varphi_{3}$,

$$
\varphi_{3}=z \text {. }
$$


- Calculamos $N$,

$$
N=\left(\frac{z+\bar{z}}{1+|z|^{2}},-i\left(\frac{z-\bar{z}}{1+|z|^{2}}\right), \frac{-1+|z|^{2}}{1+|z|^{2}}\right)
$$

- Calculamos NR,

$$
N R=N
$$

$\log o$

$$
\varphi(u, v)=2 \operatorname{Re}\left\{\int\left(\left(\frac{1}{2}-\frac{z^{2}}{2}\right),\left(\frac{i}{2}+\frac{i z^{2}}{2}\right), z\right)\right\}-\left(\frac{z+\bar{z}}{1+|z|^{2}},-i\left(\frac{z-\bar{z}}{1+|z|^{2}}\right), \frac{-1+|z|^{2}}{1+|z|^{2}}\right) .
$$

Assim, supondo $\varphi(u, v)=(x(u, v), y(u, v), z(u, v))$, temos

$$
\begin{aligned}
& x(u, v)=2 \operatorname{Re} \int\left(\frac{1}{2}-\frac{z^{2}}{2}\right) d z-\frac{z-\bar{z}}{1+|z|^{2}} \\
& =2 \operatorname{Re}\left(\frac{z}{2}-\frac{z^{3}}{6}\right)-\frac{z+\bar{z}}{1+|z|^{2}} \\
& =2 \operatorname{Re}\left(\frac{6 z-2 z^{3}}{12}\right)-\frac{z+\bar{z}}{1+|z|^{2}} \\
& =\frac{1}{6} \operatorname{Re}\left(6(u+i v)-2\left(u^{3}+3 u^{2}(i v)+3 u\left(-v^{2}\right)-i v^{3}\right)\right)-\left(\frac{2 u}{1+u^{2}+v^{2}}\right) \\
& =\frac{1}{6}\left(6 u-2 u^{3}+6 u v^{2}\right)-\frac{2 u}{1+u^{2}+v^{2}}, \\
& y(u, v)=2 \operatorname{Re} \int\left(\frac{i}{2}+\frac{i z^{2}}{2}\right) d z+i\left(\frac{z-\bar{z}}{1+|z|^{2}}\right) \\
& =2 \operatorname{Re}\left(\frac{i z}{2}+\frac{i z^{3}}{6}\right)+i\left(\frac{z-\bar{z}}{1+|z|^{2}}\right) \\
& =2 \operatorname{Re}\left(\frac{6 i z+2 i z^{3}}{12}\right)+i\left(\frac{z-\bar{z}}{1+|z|^{2}}\right) \\
& =\frac{1}{6} \operatorname{Re}\left[i\left(6(u+i v)+2\left(u^{3}+3 u^{2}(i v)+3 u\left(-v^{2}\right)-i v^{3}\right)\right)\right]-i\left(\frac{2 i v}{1+u^{2}+v^{2}}\right) \\
& =\frac{1}{6}\left(-6 v-6 u^{2} v+2 v^{3}\right)+\frac{2 v}{1+u^{2}+v^{2}}, \\
& z(u, v)=2 \operatorname{Re}\left(\int z d z\right)-\frac{-1+|z|^{2}}{1+|z|^{2}} \\
& =2 \operatorname{Re}\left(\frac{z^{2}}{2}\right)-\frac{-1+|z|^{2}}{1+|z|^{2}} \\
& =\operatorname{Re}\left((u+i v)^{2}\right)-\frac{-1+u^{2}+v 2}{1+u^{2}+v^{2}} \\
& =\operatorname{Re}\left(u^{2}+2 i u v-v^{2}\right)-\frac{-1+u^{2}+v 2}{1+u^{2}+v^{2}} \\
& =u^{2}-v^{2}-\frac{-1+u^{2}+v^{2}}{1+u^{2}+v^{2}} \text {. }
\end{aligned}
$$


Assim, temos

$$
\begin{aligned}
& x(u, v)=\frac{1}{3}\left(-u^{3}+3 u+3 u v^{2}\right)-\frac{2 u}{1+u^{2}+v^{2}} \\
& y(u, v)=\frac{1}{3}\left(v^{3}-3 v-3 u^{2} v\right)-\frac{2 v}{1+u^{2}+v^{2}} \\
& z(u, v)=u^{2}-v^{2}-\frac{u^{2}+v^{2}-1}{u^{2}+v^{2}+1}
\end{aligned}
$$

Portanto, temos a superficie-ML $S_{2}:=\varphi\left(\mathbb{R}^{2}\right)$ dada na figura a seguir,
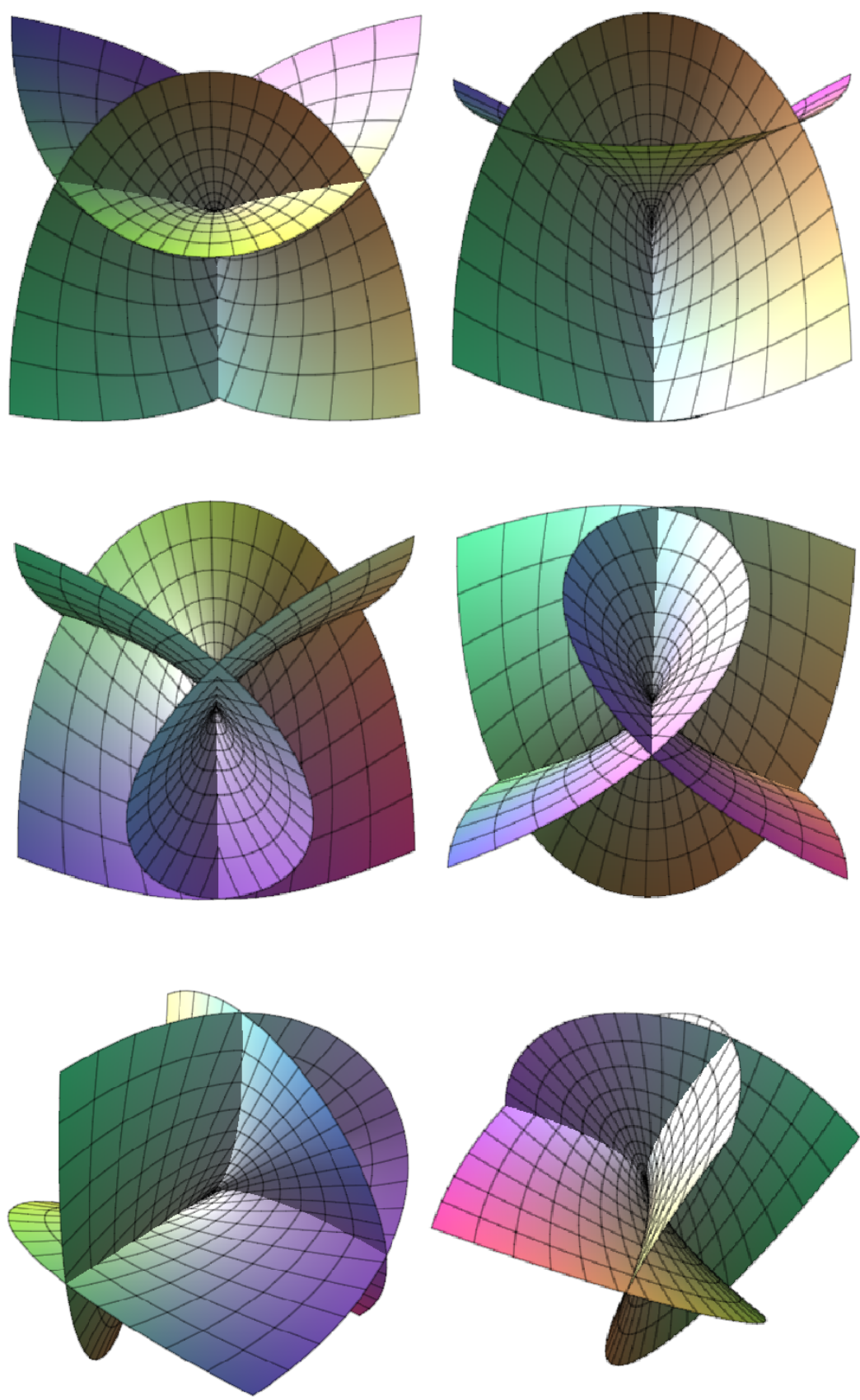
Agora, da fórmula dada na equação (3.25), temos

$$
K=\frac{4|d g|^{2}}{4 R^{2}|d g|^{2}-\left(1+|g|^{2}\right)^{2}\left|\frac{w+\partial R}{g}+\bar{g}(w-\partial R)\right|^{2}} .
$$

Já que nossos dados são

$$
g(z)=z, R(z)=1, \varphi_{3}=z d z
$$

então, temos

$$
w=z, d g=1 d z, \partial R=R_{z} d z=0
$$

substituindo na fórmula da curvatura, temos

$$
\begin{aligned}
K & =\frac{4|d z|^{2}}{4|d z|^{2}-\left(1+|z|^{2}\right)^{2}\left|\frac{z d z}{z}+\bar{z}(z d z)\right|^{2}} \\
& =\frac{4|d z|^{2}}{4|d z|^{2}-\left.\left.\left(1+|z|^{2}\right)^{2}|d z+| z\right|^{2} d z\right|^{2}} \\
& =\frac{4}{4-\left(1+|z|^{2}\right)^{2}\left(1+|z|^{2}\right)^{2}}=\frac{4}{4-\left(1+|z|^{2}\right)^{4}} \\
& =\frac{4}{4-\left(1+u^{2}+v^{2}\right)^{4}} .
\end{aligned}
$$

A superfície $\tilde{S}$ é uma superfície de Weingarten que é um exemplo de superfície-ML que não é mínima no sentido Euclidiano.

Exemplo 3.6. (Uma superficie-MGL) Sejam $g(z)=z^{2}, R(z)=1, \psi_{3}=z$.

Neste caso, temos que $d g=2 z d z, \partial R=0$ e $w=z d z$.

- Calculamos $\psi_{1}$,

$$
\begin{aligned}
2 \psi_{1} & =\frac{R_{z}+\psi_{3}}{g}+g\left(R_{z}-\psi_{3}\right) \\
& =\frac{z}{z^{2}}+z^{2}(-z) \\
& =\frac{1}{z}-z^{3} \\
& =\left(\frac{1}{z}-z^{3}\right)
\end{aligned}
$$

então,

$$
\psi_{1}=\frac{1}{2}\left(\frac{1}{z}-z^{3}\right)
$$


- Calculamos $\psi_{2}$,

$$
\begin{aligned}
2 \psi_{2} & =i\left(\frac{R_{z}+\psi_{3}}{g}\right)-i g\left(R_{z}-\psi_{3}\right) \\
& =i\left(\frac{z}{z^{2}}\right)-i z^{2}(-z) \\
& =\frac{i}{z}+i z^{3}
\end{aligned}
$$

então,

$$
\psi_{2}=\frac{i}{2}\left(\frac{1}{z}+z^{3}\right)
$$

- Já conhecemos $\psi_{3}$,

$$
\psi_{3}=z
$$

- Calculamos $N$,

$$
N=\left(\frac{z^{2}+\bar{z}^{2}}{1+|z|^{4}},-i\left(\frac{z^{2}-\bar{z}^{2}}{1+|z|^{4}}\right), \frac{-1+|z|^{4}}{1+|z|^{4}}\right)
$$

- Calculamos NR,

$$
N R=R,
$$

$\log$,

$$
\psi(u, v)=2 \operatorname{Re} \int\left\{\left(\frac{1}{2}\left(\frac{1}{z}-z^{3}\right), \frac{i}{2}\left(\frac{1}{z}+z^{3}\right), z\right)\right\} d z-\left(\frac{z^{2}+\bar{z}^{2}}{1+|z|^{4}},-i\left(\frac{z^{2}-\bar{z}^{2}}{1+|z|^{4}}\right), \frac{-1+|z|^{4}}{1+|z|^{4}}\right) .
$$

Supondo $\psi=(x(u, v), y(u, v), z(u, v))$, temos

$$
\begin{aligned}
x(u, v) & =2 \operatorname{Re} \int \frac{1}{2}\left(\frac{1}{z}-z^{3}\right) d z-\frac{z^{2}+\bar{z}^{2}}{1+|z|^{4}} \\
& =\operatorname{Re}\left(\ln z-\frac{z^{4}}{4}\right)-\frac{z^{2}-\bar{z}^{2}}{1+|z|^{4}} \\
& =\operatorname{Re}\left(\ln |z|+i \operatorname{Arg} z-\frac{\left(z^{2}\right)^{2}}{4}\right)-\frac{z^{2}+\bar{z}^{2}}{1+|z|^{4}} \\
& =\operatorname{Re}\left(\ln \left(u^{2}+v^{2}\right)+i \operatorname{Arg} z-\frac{\left(u^{2}+2 u v i-v^{2}\right)^{2}}{4}\right)-\frac{u^{2}+2 u v i-v^{2}+u^{2}-2 u v i-v^{2}}{1+|z|^{4}} \\
& =\ln \left(u^{2}+v^{2}\right)-\frac{\left(u^{2}-v^{2}\right)^{2}-4 u^{2} v^{2}}{4}-\frac{2\left(u^{2}-v^{2}\right)}{1+\left(u^{2}+v^{2}\right)^{2}},
\end{aligned}
$$




$$
\begin{aligned}
y(u, v) & =2 \operatorname{Re}\left(\frac{i}{2}\left(\frac{1}{z}+z^{3}\right) d z+i\left(\frac{z^{2}-\bar{z}^{2}}{1+|z|^{4}}\right)\right. \\
& =\operatorname{Re}\left(i\left(\ln z+\frac{z^{4}}{4}\right)\right)+i\left(\frac{z^{2}-\bar{z}^{2}}{1+|z|^{4}}\right) \\
& =\operatorname{Re}\left(i\left(\ln |z|+i \operatorname{Arg} z+\frac{\left(z^{2}\right)^{2}}{4}\right)\right)+i\left(\frac{u^{2}+2 u v i-v^{2}-u^{2}+2 u v i+v^{2}}{1+|z|^{4}}\right) \\
& =\operatorname{Re}\left(i \ln |z|-\operatorname{Arg} z+i \frac{\left(u^{2}+2 u v i-v^{2}\right)^{2}}{4}\right)+\frac{-4 u v}{1+|z|^{4}} \\
& =-\operatorname{Arg} z+\operatorname{Re}\left(\frac{i\left(\left(u^{2}-v^{2}\right)^{2}+4 u v i\left(u^{2}-v^{2}\right)-4 u^{2} v^{2}\right)}{4}\right)-\frac{4 u v}{1+|z|^{4}} \\
& =-\arctan \left(\frac{v}{u}\right)-u v\left(u^{2}-v^{2}\right)-\frac{4 u v}{1+\left(u^{2}+v^{2}\right)^{2}}, \\
z(u, v) & =2 \operatorname{Re} \in z d z-\frac{-1+|z|^{4}}{1+|z|^{4}} \\
& =2 \operatorname{Re}\left(\frac{z^{2}}{2}\right)-\frac{-1+|z|^{4}}{1+|z|^{4}} \\
& =\operatorname{Re}\left(u^{2}+2 u v i-v^{2}\right)-\frac{-1+\left(u^{2}+v^{2}\right)^{2}}{1+\left(u^{2}+v^{2}\right)^{2}} \\
& =\left(u^{2}-v^{2}\right)-\frac{-1+\left(u^{2}+v^{2}\right)^{2}}{1+\left(u^{2}+v^{2}\right)^{2}}
\end{aligned}
$$

Assim,

$$
\begin{aligned}
& x(u, v)=\ln \left(u^{2}+v^{2}\right)-\frac{\left(u^{2}-v^{2}\right)^{2}-4 u^{2} v^{2}}{4}-\frac{2\left(u^{2}-v^{2}\right)}{1+\left(u^{2}+v^{2}\right)^{2}}, \\
& y(u, v)=-\arctan \left(\frac{v}{u}\right)-u v\left(u^{2}-v^{2}\right)-\frac{4 u v}{1+\left(u^{2}+v^{2}\right)^{2}}, \\
& z(u, v)=\left(u^{2}-v^{2}\right)-\frac{-1+\left(u^{2}+v^{2}\right)^{2}}{1+\left(u^{2}+v^{2}\right)^{2}} .
\end{aligned}
$$

Portanto, temos a superficie-MGL $S_{3}:=\psi\left(\mathbb{R}^{2}\right)$ dada na figura a seguir,


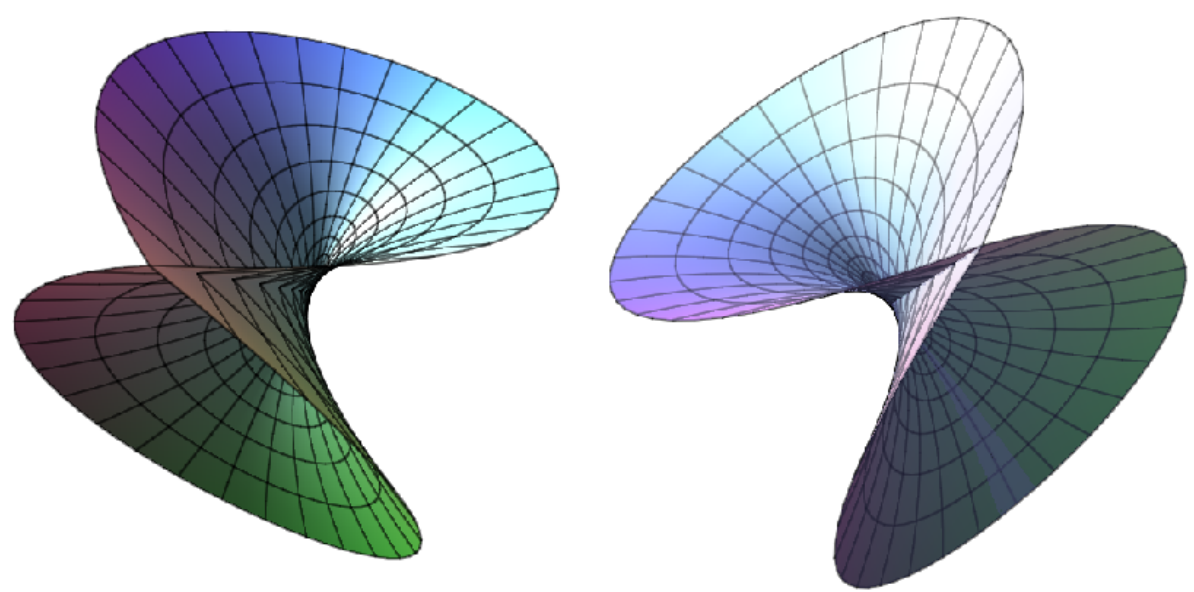

Logo, calculamos a curvatura Gaussiana,

$$
\begin{aligned}
K & =\frac{4|2 z d z|^{2}}{4|2 z d z|^{2}-\left(1+\left|z^{2}\right|^{2}\right)^{2}\left|\frac{z d z}{z^{2}}+\bar{z}^{2}(z d z)\right|^{2}} \\
& =\frac{16|z|^{2}|d z|^{2}}{16\left|z^{2}\right||d z|^{2}-\left(1+|z|^{4}\right)^{2}\left|\frac{1}{z}+\bar{z}^{2} \cdot z\right|^{2}|d z|^{2}} \\
& =\frac{16|z|^{2}}{16|z|^{2}-\frac{\left(1+|z|^{4}\right)^{2}\left(1+|z|^{4}\right)^{2}}{|z|^{2}}} \\
& =\frac{16|z|^{4}}{16|z|^{4}-\left(1+|z|^{4}\right)^{4}} \\
& =\frac{16\left(u^{2}+v^{2}\right)}{16\left(u^{2}+v^{2}\right)^{2}-\left(1+\left(u^{2}+v^{2}\right)^{2}\right)^{4}} .
\end{aligned}
$$

Em $u=v=0$, temos que $K=0$.

\subsection{Completude da métrica de Laguerre}

Seja $\psi$ uma superfície-ML em $\mathbb{R}^{3}$. Pelo visto no Capítulo 2, sabe-se que a forma quadrática

$$
h_{L}=\frac{H^{2}-K}{K^{2}} I I I
$$

é invariante pelo grupo de Laguerre e, de fato, é uma métrica Riemanniana se $\psi$ não tem pontos umbílicos, isto é, se $H^{2}-K \neq 0$. De (2.78), temos que $h_{L}$ é a métrica de Laguerre.

Das expressões (3.21) e (3.25), a métrica de Laguerre pode-se expressar como

$$
h_{L}=\frac{H^{2}-K}{K^{2}} I I I=\left(R^{2}-\frac{1}{K}\right) I I I=\left|\frac{\omega+\partial R}{g}+\bar{g}(\omega-\partial R)\right|^{2} .
$$


No seguinte estudaremos a relação entre a completude Euclidiana de uma imersão e a completude da métrica de Laguerre.

Teorema 3.3. Seja $\psi: S \rightarrow \mathbb{R}^{3}$ uma superfície completa com curvatura negativa. Então a métrica de Laguerre $h_{L}$ de $\psi$ é completa.

Demonstração. Seja $p$ um ponto de $S$. Como $K(p)<0$, existem coordenadas doblemente ortogonais $(u, v)$ em um entorno de $p$ tal que a primeira forma fundamental e a segunda forma fundamental de $\psi$ estão dadas por

$$
I=E d u^{2}+G d v^{2}, \quad I I=k_{1} E d u^{2}+k_{2} G d v^{2}
$$

onde $E, G$ são funções positivas e $k_{1}, k_{2}$ são curvaturas principais.

Então, a terceira forma fundamental pode-se escrever como $I I I=k_{1}^{2} E d u^{2}+k_{2}^{2} G d v^{2}$ e a métrica de Laguerre como

$$
\begin{aligned}
h_{L} & =\frac{H^{2}-K}{K^{2}} I I I=\frac{1}{4}\left(\frac{1}{k_{1}}-\frac{1}{k_{2}}\right)^{2}\left(k_{1}^{2} E d u^{2}+k_{2}^{2} G d v^{2}\right) \\
& =\frac{1}{4}\left(\left(1-\frac{k_{1}}{k_{2}}\right)^{2} E d u^{2}+\left(1-\frac{k_{2}}{k_{1}}\right)^{2} G d v^{2}\right) \geq \frac{1}{4} I
\end{aligned}
$$

onde foi utilizado que $k_{1} k_{2}<0$.

Esta desigualdade mostra que a completude da métrica Euclidiana $I$, implica que a métrica de Laguerre é também completa.

Se $\psi: S \rightarrow \mathbb{R}^{3}$ é uma superfície-MGL não plana com curvatura Gaussiana $K \leq 0$, é bom aclarar que a métrica de Laguerre é uma métrica bem definida sobre o conjunto dos pontos isolados $K=0$. Para isso, observe que, de (3.39), $h_{L}$ é uma forma quadrática bem definida em qualquer superfície-MGL pois $(\omega+\partial R) / g$ e $g(w-\partial R)$ são 1 -formas holomorfas (Teorema 3.1). Além disso, se $p \in S \operatorname{com} K(p)=0$ então existe um entorno de $p$, exceto o próprio ponto $p$, onde $K<0$. Portanto, de (3.40), $I \leq 4 h_{L}$ neste entorno, e assim $I(p) \leq 4 h_{L}(p)$. Portanto, $h_{L}$ é também uma métrica Riemanniana em $p$.

Por outro lado, é importante esclarecer que o resultado recíproco do Teorema 3.3 não é verdadeiro, isto é, a completude de Laguerre não implica a completude Euclidiana. Neste sentido, mostraremos o seguinte exemplo.

Exemplo 3.7. Consideremos a superfície-ML dada pelo Teorema 3.1 para $S=\mathbb{C} \equiv \mathbb{R}^{2}$ com

$$
R=\frac{z^{2}+\bar{z}^{2}}{2}, \quad \omega=-z d z, \quad g(z)=-\frac{1}{z}, \quad z \in \mathbb{C} .
$$

Temos $d g=\frac{1}{z^{2}} d z$ e $d R=z d z+\bar{z} d z$, de onde $\partial R=z d z$.

- Calculamos $\phi_{1}$,

$$
2 \phi_{1}=-\frac{1}{z}(z+z) d z=-2 d z
$$

$\log o$

$$
\phi_{1}=-d z
$$

- Calculamos $\phi_{2}$,

$$
2 \phi_{2}=\frac{i}{z}(z+z) d z
$$


$\log o$

$$
\phi_{2}=i
$$

- Conhecendo $\omega$, já conhecemos $\phi_{3}$,

$$
\phi_{3}=-z
$$

- Calculando $N$,

$$
\begin{aligned}
N & =\left(\frac{-\frac{1}{z}-\frac{1}{\bar{z}}}{1+\left|\frac{1}{z}\right|^{2}},-i\left(\frac{-\frac{1}{z}+\frac{1}{\bar{z}}}{1+\left|\frac{1}{z}\right|^{2}}\right), \frac{-1+\left|\frac{1}{z}\right|^{2}}{1+\left|\frac{1}{z}\right|^{2}}\right) \\
& =\left(\frac{-(z+\bar{z})}{|z|^{2}+1}, \frac{-i(z-\bar{z})}{|z|^{2}+1}, \frac{-|z|^{2}+1}{|z|^{2}+1}\right) .
\end{aligned}
$$

- Calculamos NR,

$$
N R=\left(-\frac{1}{2}\left(\frac{(z+\bar{z})\left(z^{2}+\bar{z}^{2}\right)}{2\left(|z|^{2}+1\right)}\right),-\frac{i}{2}\left(\frac{(z-\bar{z})\left(z^{2}+\bar{z}^{2}\right)}{2\left(|z|^{2}+1\right)}\right), \frac{\left(-|z|^{2}+1\right)\left(z^{2}+\bar{z}^{2}\right)}{2\left(|z|^{2}+1\right)}\right)
$$

Logo, supondo $\phi=(x, y, z)$, temos

$$
\begin{aligned}
x(u, v) & =2 \operatorname{Re} \int(-1) d z+\frac{1}{2} \frac{(z+\bar{z})\left(z^{2}+\bar{z}^{2}\right)}{\left(|z|^{2}+1\right)} \\
& =-2 u+\frac{1}{2} \frac{(2 u) 2\left(u^{2}-v^{2}\right)}{\left(u^{2}+v^{2}+1\right)} \\
& =-2 u\left(1-\frac{u^{2}-v^{2}}{u^{2}+v^{2}+1}\right) \\
& =-2 u\left(\frac{1+2 v^{2}}{u^{2}+v^{2}+1}\right), \\
y(u, v) & =2 \operatorname{Re} \int i d z+\frac{i}{2}\left(\frac{(z-\bar{z})\left(z^{2}+\bar{z}^{2}\right)}{\left(|z|^{2}+1\right)}\right) \\
& =2 \operatorname{Re}(i z)+\frac{i}{2} \frac{(2 v i) 2\left(u^{2}-v^{2}\right)}{\left(u^{2}+v^{2}+1\right)} \\
& =-2 v-\frac{2 v\left(u^{2}-v^{2}\right)}{u^{2}+v^{2}+1}=-2 v\left(1+\frac{u^{2}-v^{2}}{u^{2}+v^{2}+1}\right) \\
& =-2 v\left(\frac{1+2 u^{2}}{u^{2}+v^{2}+1}\right),
\end{aligned}
$$


3.3 Completude da métrica de Laguerre

72

$$
\begin{aligned}
z(u, v) & =-2 \operatorname{Re} \int z d z-\frac{\left(-|z|^{2}+1\right)\left(z^{2}+\bar{z}^{2}\right)}{2\left(|z|^{2}+1\right)} \\
& =-2 \operatorname{Re}\left(\frac{z^{2}}{2}\right)-\frac{\left(-u^{2}-v^{2}+1\right)\left(u^{2}-v^{2}\right)}{\left(u^{2}+v^{2}+1\right)} \\
& =-\left(u^{2}-v^{2}\right)+\frac{\left(u^{2}+v^{2}-1\right)\left(u^{2}-v^{2}\right)}{u^{2}+v^{2}+1} \\
& =\frac{-\left(u^{2}-v^{2}\right)\left(u^{2}+v^{2}+1\right)+\left(u^{2}-v^{2}\right)\left(u^{2}+v^{2}-1\right)}{u^{2}+v^{2}+1} \\
& =\frac{-\left(u^{2}-v^{2}\right)-\left(u^{2}-v^{2}\right)}{u^{2}+v^{2}+1} \\
& =\frac{-2 u^{2}+2 v^{2}}{u^{2}+v^{2}+1} .
\end{aligned}
$$

Então,

$$
\phi(u, v)=\left(\frac{-2 u\left(1+2 v^{2}\right)}{1+u^{2}+v^{2}}, \frac{-2 v\left(1+2 u^{2}\right)}{1+u^{2}+v^{2}}, \frac{-2 u^{2}+2 v^{2}}{1+u^{2}+v^{2}}\right) .
$$

Aliás, a superficie tem curvatura

$$
K=-\frac{1}{2\left(u^{2}+v^{2}\right)+4 u^{2} v^{2}+1} .
$$

Portanto, temos a superfície-ML $\phi\left(\mathbb{R}^{2}\right)$ dada na figura a seguir.
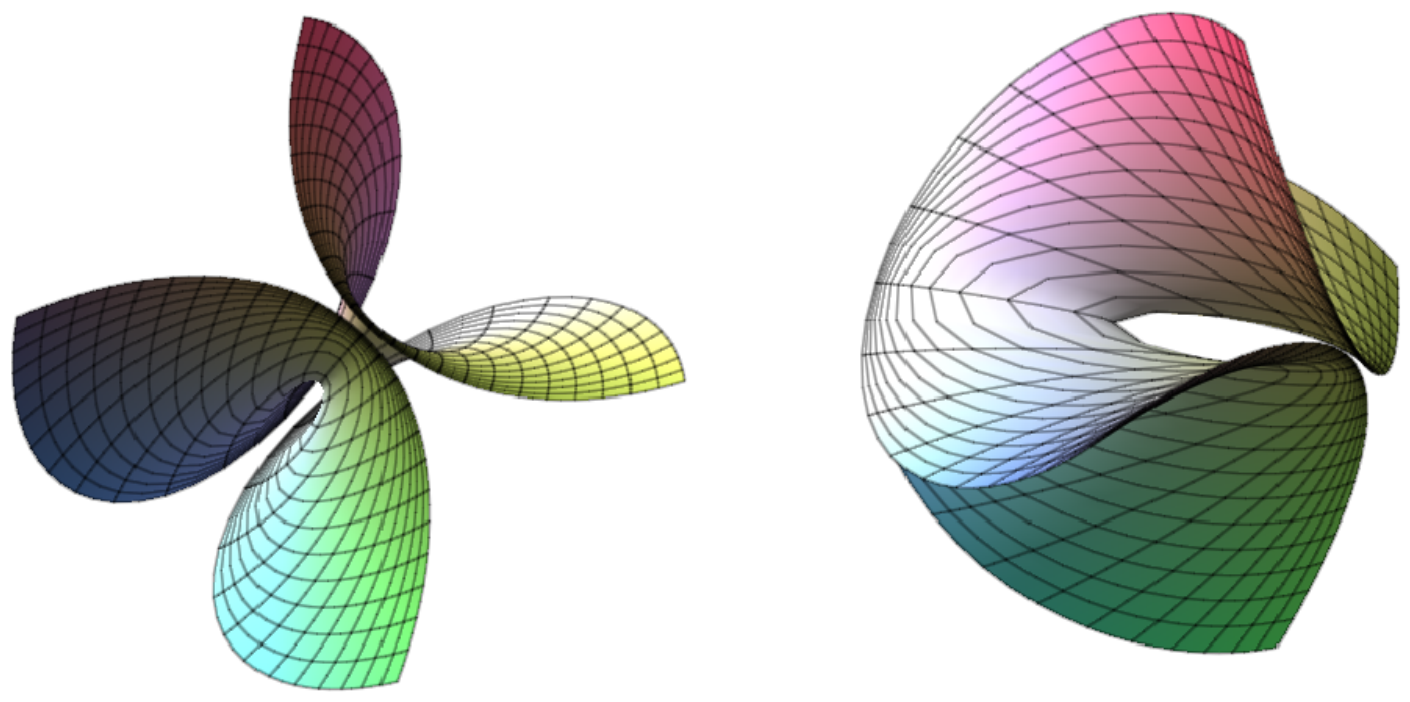

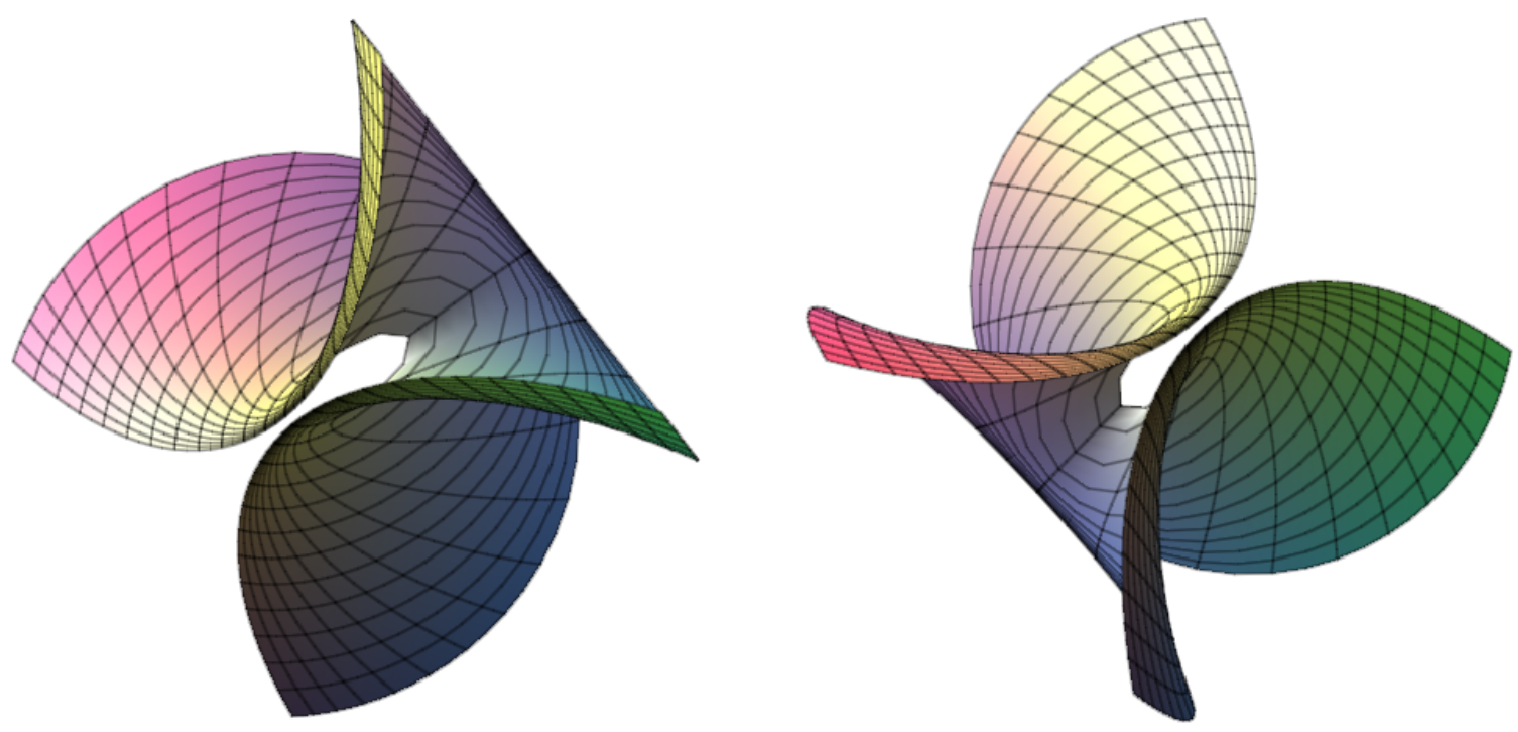

Como $\psi:=(X(u, v), Y(u, v), Z(u, v))$, temos

$$
\begin{aligned}
& x_{u}=-\frac{2\left(1+v^{2}\right)}{1+u^{2}+v^{2}}+\frac{4 u^{2}\left(1+2 v^{2}\right)}{\left(1+u^{2}+v^{2}\right)^{2}}, \\
& y_{u}=-\frac{8 u v}{1+u^{2}+v^{2}}+\frac{4 u v\left(1+2 u^{2}\right)}{\left(1+u^{2}+v^{2}\right)^{2}}, \\
& z_{u}=-\frac{4 u}{1+u^{2}+v^{2}}-\frac{2 u\left(-2 u^{2}+2 v^{2}\right)}{\left(1+u^{2}+v^{2}\right)^{2}},
\end{aligned}
$$

$e$

$$
\begin{aligned}
& x_{v}=-\frac{8 u v}{1+u^{2}+v^{2}}+\frac{4 u v\left(1+2 v^{2}\right)}{\left(1+u^{2}+v^{2}\right)^{2}}, \\
& y_{v}=-\frac{2\left(1+2 u^{2}\right)}{u^{2}+v^{2}+1}+\frac{4 v^{2}\left(1+2 u^{2}\right)}{\left(1+u^{2}+v^{2}\right)^{2}}, \\
& z_{v}=\frac{4 v}{1+u^{2}+v^{2}}-\frac{2 v\left(-2 u^{2}+2 v^{2}\right)}{\left(1+u^{2}+v^{2}\right)^{2}} .
\end{aligned}
$$

Então,

$$
\begin{aligned}
& E=\left\langle\psi_{u}, \psi_{u}\right\rangle=\frac{4\left(1+2 v^{2}\right)^{2}}{\left(1+u^{2}+v^{2}\right)^{2}}, \\
& F=\left\langle\psi_{u}, \psi_{v}\right\rangle=0, \\
& G=\left\langle\psi_{v}, \psi_{v}\right\rangle=\frac{4\left(1+2 u^{2}\right)}{\left(1+u^{2}+v^{2}\right)^{2}} .
\end{aligned}
$$

Assim, temos a primeira forma fundamental

$$
I=\frac{4\left(1+2 v^{2}\right)}{\left(1+u^{2}+v^{2}\right)^{2}} d u^{2}+\frac{4\left(1+2 u^{2}\right)^{2}}{\left(1+u^{2}+v^{2}\right)^{2}} d v^{2},
$$


e a métrica de Laguerre dada por

$$
\begin{aligned}
g & =\left|\frac{w+\partial R}{g}+\bar{g}(w-\partial R)\right|^{2} \\
& =\left|-\frac{1}{z}(-z d z-z d z)\right|^{2} \\
& =\left|\frac{2 z d z}{\bar{z}}\right|^{2} \\
& =4|d z|^{2} \\
& =4\left(d u^{2}+d v^{2}\right) .
\end{aligned}
$$

Note que, tomando a curva $\alpha(t)=\psi(t, 0)=\left(\frac{-2 t}{1+t^{2}}, \frac{-2 t^{2}}{1+t^{2}}\right)$, temos

$$
\begin{aligned}
\alpha^{\prime}(t) & =\left(\frac{-2\left(1+t^{2}\right)+2 t(2 t)}{\left(1+t^{2}\right)^{2}}, 0, \frac{-4 t\left(1+t^{2}\right)+2 t^{2}(2 t)}{\left(1+t^{2}\right)^{2}}\right) \\
& =\left(\frac{-2+2 t^{2}}{\left(1+t^{2}\right)^{2}}, 0, \frac{-4 t}{\left(1+t^{2}\right)^{2}}\right) \\
& =2\left(\frac{-1+t^{2}}{\left(1+t^{2}\right)^{2}}, 0, \frac{-2 t}{\left(1+t^{2}\right)^{2}}\right),
\end{aligned}
$$

então,

$$
\begin{aligned}
\left|\alpha^{\prime}(t)\right|^{2} & =4\left(\frac{1-2 t^{2}+t^{4}+4 t^{2}}{\left(1+t^{2}\right)^{4}}\right) \\
& =4\left(\frac{1+2 t^{2}+t^{4}}{\left(1+t^{2}\right)^{4}}\right) \\
& =4\left(\frac{1}{\left(1+t^{2}\right)^{2}}\right)
\end{aligned}
$$

$\log o$

$$
\left|\alpha^{\prime}(t)\right|=\frac{2}{1+t^{2}}
$$

Tomando a longitude de a temos

$$
l(\alpha)=\int_{0}^{\infty} \frac{2}{\left(1+t^{2}\right)} d t=\left.2 \arctan (t)\right|_{0} ^{\infty}=2\left(\frac{\pi}{2}\right)=\pi<\infty
$$

que demonstra que a métrica induzida I não é completa. 


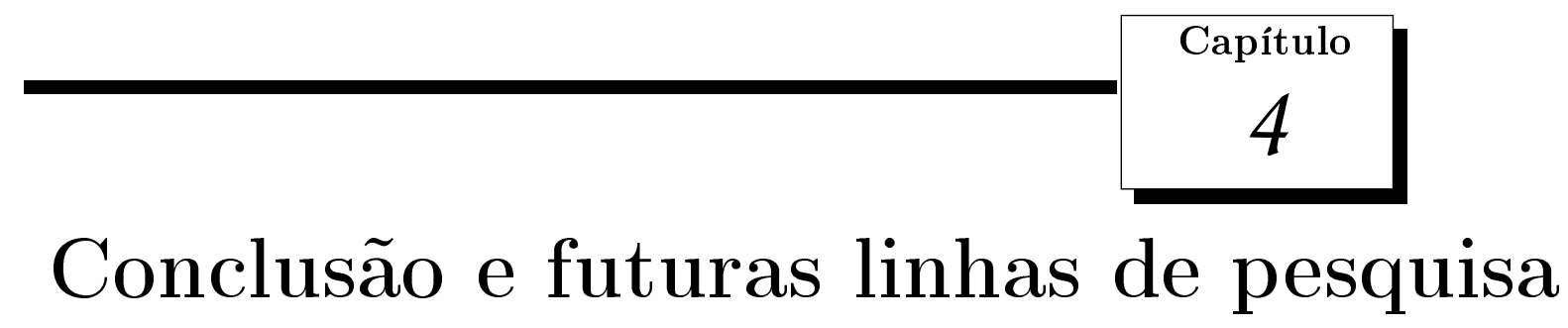

No presente trabalho apresentamos a geometria de Laguerre, através de planos e esferas orientadas e, neste contexto, apresentamos a métrica e o funcional de Laguerre. Em seguida, apresentamos uma maneira de representar as superfícies-ML em $\mathbb{R}^{3}$, que são pontos críticos do funcional de Laguerre, de forma global usando três dados: uma função meromorfa, uma forma holomorfa e uma função real harmônica. Esta representação permitiu construir vários exemplos de superfícies mínimas de Laguerre generalizadas, isto é, aquelas superfícies mínimas de Laguerre que possuem curvatura zero em pontos isolados.

Por outro lado, ainda existem trabalhos nesta linha de pesquisa que obtém resultados envolvendo superfíciesML. A saber, no artigo [21], cada superfície-ML $x: M \rightarrow \mathbb{R}^{3}$ com $\Omega^{*} \not \equiv 0$ (ver [21], § 2) é associada injetivamente a uma solução complexa da equação de Liouville em um aberto $U$,

$$
\Delta f=e^{f}, \quad f: U \subset M \rightarrow \mathbb{C} .
$$

E já que as soluções da equação de Liouville são dadas por

$$
f=\log \frac{8 \phi_{z} \bar{\psi}_{z}}{(\phi+\bar{\psi})^{2}}
$$

temos que qualquer superfície-ML em $\mathbb{R}^{3}$ com $\Omega^{*} \not \equiv 0$ é determinada por duas funções holomorfas.

Além dessas representações, no artigo [20] é apresentada uma maneira de construir uma superfície-ML usando congruências de retas, citamos o Teorema central do artigo

Teorema 4.1. Seja $\Sigma$ uma congruência de retas dada por $(\xi, F(\xi))$. Se $F$ é harmônica, então a envoltória dos planos médios $M_{\Sigma}$ é uma superfície-ML nos pontos suaves. Reciprocamente, qualquer superfície-ML pode ser localmente descrita como a envoltória dos planos médios de uma congruência tal que $F(\xi)$ seja harmônica.

Neste último caso, surgiu um estudo das superfícies geradas a partir da superfície média de $M_{\Sigma}$, a idéia é a seguinte: 
Considerando uma superfície-ML $S$, parametrizada por $X: M \rightarrow \mathbb{R}^{3}$, descrevemos sua superfície média por $Y=X+\left(\frac{H}{K}\right) N$, sendo que $\Delta^{I I I}\left(\frac{H}{K}\right)=0$, localmente existe la harmônica conjugada $\left(\frac{H}{K}\right)^{*}$, assim, podemos descrever duas superficies $M_{1}$ e $M_{2}$ parametrizadas por $Z_{1}=Y+\left(\frac{H}{K}\right)^{*} N$ e $Z_{2}=Y-\left(\frac{H}{K}\right)^{*} N$ respectivamente e uma aplicação $T: M_{1} \rightarrow M_{2}$ que associa de forma "natural" pontos das superfícies $M_{1}$ e $M_{2}$. Algumas questões sobre tais superfícies são

1. A aplicação T preserva áreas?

2. As superficies $M_{1}$ e $M_{2}$ diferem apenas por uma reflexão?

3. As curvaturas Gaussianas das superfícies $M_{1}$ e $M_{2}$ são negativas?

4. Sobre quais condições $M_{1}$ e $M_{2}$ são superfícies-ML?

Mesmo não tendo os resultados formais, usando métodos computacionais pode-se verificar para uma grande quantidade de exemplos que a resposta à questão 2 é verdadeira quando são tomadas ao acaso duas funções $A, B$ que definem uma congruência de retas (ver [20], pg.7). A seguir, apresentamos alguns exemplos e os respectivos desenhos de $M_{1}$ e $M_{2}$, dando uma forte impressão de simetria.
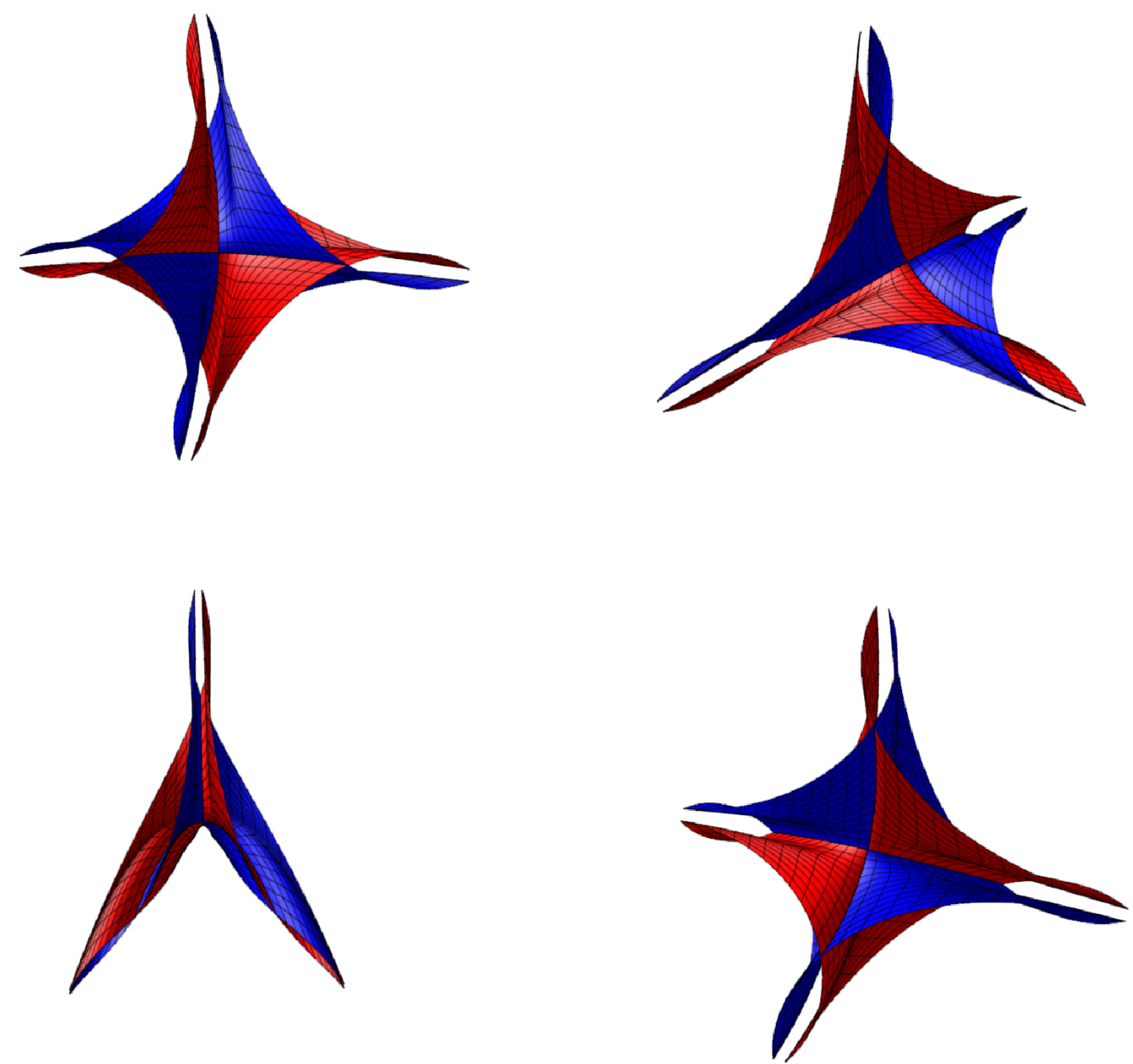

$A=0, B=z$ 

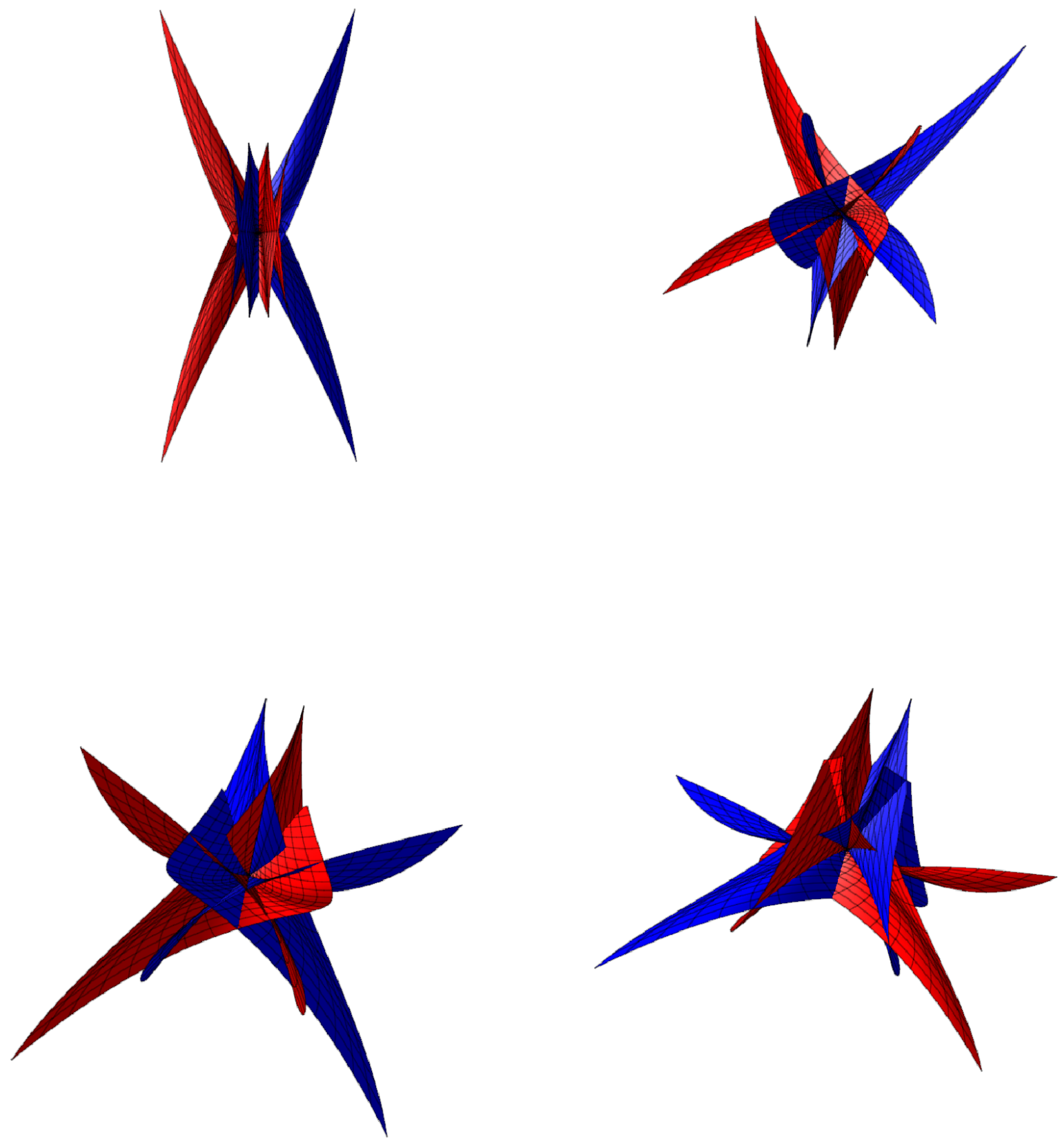

$A=1, B=z^{2}$ 



$$
A=e^{z}, B=z
$$



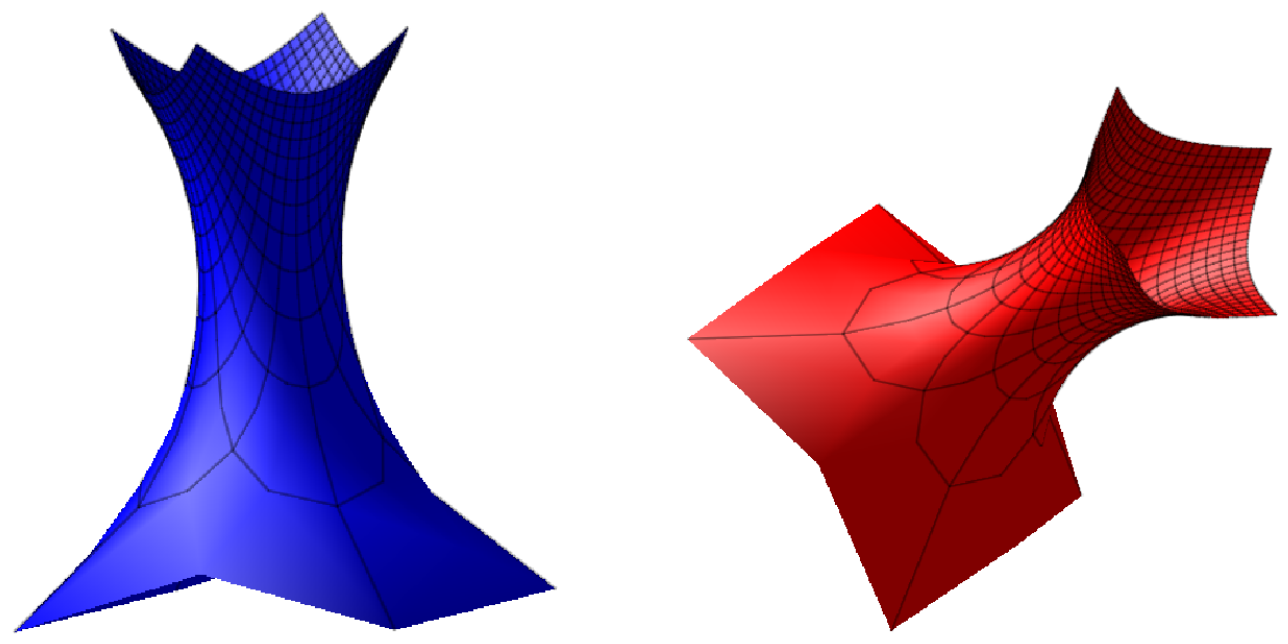

$$
A=z \log z, B=0
$$

Neste último caso pode-se ver que a superfície-ML é como caso particular uma superfície mínima e aliás, $M_{1}=M_{2}$. De maneira intuitiva também pode-se dar uma resposta à questão 3. 


\section{Apêndice}

\section{Superfícies}

\section{A.1 Demonstrações de resultados anteriores}

\section{A.1.1 Demonstração do Teorema 1.1}

Teorema 1.1 Seja $M^{m}$ uma subvariedade diferenciável e $(U, X)$ um sistema de coordenadas em $M$. Dada $f \in \mathcal{D}(M)$, denote a representação correspondente de $f$ por $\widehat{f}:=f \circ X^{-1}: X(U) \rightarrow \mathbb{R}$ e seja $\left(g^{i j}\right)$ a matriz inversa de $\left(g_{i j}\right)=\left(g_{p}\left(\frac{\partial}{\partial x_{i}}, \frac{\partial}{\partial x_{j}}\right)\right)$. Então no dominio $U$ temos

$$
\operatorname{grad} f=\sum_{i, j=1}^{m} g^{i j} \frac{\partial f}{\partial x_{i}} \cdot \frac{\partial}{\partial x_{j}}=\sum_{i, j=1}^{m} g^{i j}\left(\frac{\partial \widehat{f}}{\partial x_{i}} \circ X\right) \cdot \frac{\partial}{\partial x_{j}}
$$

Demonstração. Calculamos, para $\alpha \in\{1, \cdots, m\}$,

$$
\begin{aligned}
d f_{p}\left(\frac{\partial}{\partial x_{\alpha}}\right) & =\frac{\partial \widehat{f}}{\partial x_{\alpha}}(X(p)) \\
& =\frac{\partial\left(f \circ X^{-1}\right)}{\partial x_{\alpha}}((X(p)) \\
& =\sum_{i=1}^{m} \frac{\partial\left(f \circ X^{-1}\right)}{\partial x_{i}}\left((X(p)) \delta_{i \alpha}\right. \\
& =\sum_{i, j=1}^{m} \frac{\partial\left(f \circ X^{-1}\right)}{\partial x_{i}}\left((X(p)) g^{i j}(p) g_{j \alpha}(p)\right. \\
& =\sum_{i, j=1}^{m} \frac{\partial\left(f \circ X^{-1}\right)}{\partial x_{i}}\left((X(p)) g^{i j}(p) g_{p}\left(\frac{\partial}{\partial x_{j}}, \frac{\partial}{\partial x_{\alpha}}\right)\right. \\
& =g_{p}\left(\sum_{i, j=1}^{m} g^{i j}(p) \frac{\partial \widehat{f}}{\partial x_{i}}(X(p)) \cdot \frac{\partial}{\partial x_{j}}, \frac{\partial}{\partial x_{\alpha}}\right) .
\end{aligned}
$$


Por outro lado, da definição do gradiente, temos

$$
g_{p}\left(\operatorname{grad} f(p), \frac{\partial}{\partial x_{\alpha}}\right)=d f_{p}\left(\frac{\partial}{\partial x_{\alpha}}\right)
$$

De onde segue o resultado.

\section{A.1.2 Demonstração do Lema 1.1}

Lema 1.1 Seja $F:(-\varepsilon, \varepsilon) \rightarrow G L(n, \mathbb{C})$ uma curva diferenciável no grupo das matrizes invertiveis. Então

$$
\operatorname{Tr}\left(F^{-1}(s) \circ F^{\prime}(s)\right)=\frac{d}{d s} \ln (\operatorname{det} F(s))
$$

Demonstração. Seja $B \in \mathbb{C}^{n \times n}$, note a expressão do seguinte determinante

$$
\operatorname{det}\left(I_{n}+t B\right)=\left|\left(\begin{array}{cccc}
1+t b_{11} & t b_{12} & \cdots & t b_{1 n} \\
t b_{21} & 1+t b_{22} & \ldots & \vdots \\
\vdots & \vdots & \ddots & \vdots \\
t b_{n 1} & \ldots & b_{n n-1} & 1+t b_{n n}
\end{array}\right)\right|=1+t \operatorname{Tr} B+O\left(t^{2}\right) .
$$

Considere agora $A \in G L(n, \mathbb{C})$ e $H \in \mathbb{C}^{n \times n}$ qualquer, então

$$
\begin{aligned}
\lim _{t \rightarrow 0} \frac{\operatorname{det}(A+t H)-\operatorname{det}(A)}{t} & =\operatorname{det}(A) \cdot \lim _{t \rightarrow 0} \frac{\operatorname{det}\left(I_{n}+t A^{-1} H\right)-1}{t} \\
& \stackrel{A .1}{=} \operatorname{det}(A) \cdot \lim _{t \rightarrow 0} \frac{t \operatorname{Tr}\left(A^{-1} H\right)+O\left(t^{2}\right)}{t} \\
& =\operatorname{det}(A) \cdot \operatorname{Tr}\left(A^{-1} H\right) .
\end{aligned}
$$

Agora consideramos a função $f(t)=\operatorname{det}\left(F(s)+t F^{\prime}(s)\right)$. Derivamos e obtemos

$$
f^{\prime}(0)=\lim _{t \rightarrow 0} \frac{\operatorname{det}\left(F(s)-t F^{\prime}(s)\right)-\operatorname{det}(F(s))}{t} \stackrel{A .2}{=} \operatorname{det}(F(s)) \cdot \operatorname{Tr}\left(F^{-1}(s) \circ F^{\prime}(s)\right) .
$$

Finalmente, a regra da cadeia implica que $f^{\prime}(0)=d(\text { det })_{F(s)}\left(F^{\prime}(s)\right)$ e

$$
\begin{aligned}
\left.\frac{d}{d t} \ln (\operatorname{det}(F(t)))\right|_{t=s} & =\left.\frac{1}{\operatorname{det}(F(s))} \cdot \frac{d}{d t}(\operatorname{det}(F(t)))\right|_{t=s} \\
& =\frac{1}{\operatorname{det}(F(s))} \cdot d(\operatorname{det})_{F(s)}\left(F^{\prime}(s)\right) \\
& =\frac{f^{\prime}(0)}{\operatorname{det}(F(s))} \\
& =\operatorname{Tr}\left(F^{-1}(s) \cdot F^{\prime}(s)\right)
\end{aligned}
$$




\section{A.1.3 Demonstração do Teorema 1.4}

Teorema 1.4 Seja $M$ uma subvariedade, sejam $f, h \in \mathcal{D}(M)$ e $\mathcal{X} \in \mathfrak{X}(M)$. Então

1. $\operatorname{grad}(f \cdot h)=f \cdot \operatorname{grad}(h)+h \cdot \operatorname{grad}(f)$,

2. $\operatorname{div}(f \cdot \mathcal{X})=f \cdot \operatorname{div}(\mathcal{X})+\mathcal{X}(f)$,

3. $\Delta(f \cdot h)=f \cdot \Delta h+h \cdot \Delta f+2\langle\operatorname{grad} f, \operatorname{grad} h\rangle$.

Demonstração.

1. Seja $\mathcal{Y} \in \mathfrak{X}(M)$ um campo qualquer e $p \in M$. Então para a derivada direcional vale

$$
g_{p}(\operatorname{div}(f \cdot h), \mathcal{Y})=\mathcal{Y}(f \cdot h)+f \cdot \mathcal{Y}(h)=g_{p}(f \cdot \operatorname{grad} h+h \cdot \operatorname{grad} f, \mathcal{Y}) .
$$

De onde se segue o resultado.

2. Segundo as hipóteses do Teorema 1.2, temos $f \mathcal{X}=\sum_{i=1}^{n}\left(f \xi_{i}\right) \frac{\partial}{\partial u_{i}}$, dai

$$
\begin{aligned}
\operatorname{div}(f \mathcal{X}) & =\frac{1}{\sqrt{\theta}} \sum_{i=1}^{n} \frac{\partial}{\partial u_{i}}\left(\left(f \xi_{i}\right) \sqrt{\theta}\right) \\
& =\frac{1}{\sqrt{\theta}} \sum_{i=1}^{n}\left\{\frac{\partial f}{\partial u_{i}}\left(\xi_{i} \sqrt{\theta}\right)+f \frac{\partial\left(\xi_{i} \sqrt{\theta}\right)}{\partial u_{i}}\right\} \\
& =\sum_{i=1}^{n} \xi_{i}\left(\frac{\partial f}{\partial u_{i}}\right)+f \operatorname{div} \mathcal{X} \\
& =\mathcal{X}(f)+f \cdot \operatorname{div} \mathcal{X} .
\end{aligned}
$$

3. Da definição do Laplaciano, temos

$$
\begin{aligned}
\Delta(f \cdot h) & =\operatorname{div}(\operatorname{grad}(f \cdot h)) \\
& \stackrel{1}{=} \operatorname{div}(h \cdot \operatorname{grad} f+f \cdot \operatorname{grad} h) \\
& \stackrel{2}{=} h \cdot \Delta f+\operatorname{grad} f(h)+f \cdot \Delta h+\operatorname{grad} h(f) \\
& =2\langle\operatorname{grad} h, \operatorname{grad} f\rangle+h \cdot \Delta f+f \cdot \Delta h .
\end{aligned}
$$




\section{Referências Bibliográficas}

[1] Aledo, Juan A., Gálvez, José A., Lozano, Victorino: Complete Laguerre minimal surfaces in $\mathbb{R}^{3}$, Elseiver, Nonlinear Analysis 92, 1-12, 2013.

[2] Bianchi, L.: Opere, v. XI, Corrispondenza, Cremonese, Roma, 199-202, 1957.

[3] Blaschke, W.: Uber die Geometrie von Laguerre: I. Grundformeln der Flächentheorie, Abh. Math. Sem. Univ. Hamburg 3, 176-194, 1924.

[4] Blaschke, W.: Uber die Geometrie von Laguerre: II. Fl, achentheorie in Ebenenkoordinaten., Abh. Math. Sem. Univ. Hamburg 3, 195-212, 1924.

[5] Blaschke, W.: Uber die Geometrie von Laguerre: III. Beitr, age zur Flächentheorie, Abh. Math. Sem. Univ. Hamburg 4, 1-12, 1925.

[6] Blaschke, W.: Vorlesungen über Differentialgeometrie, Volume 3, Springer, Berlin Heidelberg, New York, 1929.

[7] Cecil, T. E., Chern, S. S.: Dupin Submanifolds in Lie Sphere Geometry, Lecture Notes in Math. Springer, Berlin, 1369, 1-48, 1989.

[8] Cecil, T. E.: Lie Sphere Geometry: With Applications to Submanifolds, Springer, New York, 1992.

[9] Do Carmo, M.: Differential forms and applications. Springer-Verlag, Berlin, 1994.

[10] Do Carmo, M.: Geometria Riemanniana, 5ạ edição. Projeto Euclides, IMPA, Rio de Janeiro, 2005.

[11] Do Carmo, M.: Superficies Minimas, $3^{a} \underline{a}$ impressão, $2^{a}$ edição. Publicações Matemáticas, IMPA, Rio de Janeiro, 2009.

[12] Li, T. Z., Wang, C.: Laguerre geometry of hypersurfaces in $\mathbb{R}^{n}$, Manuscripta Math., 69, 73-95, 2007.

[13] Li, T. Z.: Laguerre geometry of surfaces in $\mathbb{R}^{3}$, Acta Math. Sin. 21, n 6 1525-1534, 2005.

[14] Milnor, T.K.: Abstract Weingarten Surfaces, J. Differential Geom., Volume 15, 3, 365-380, 1980.

[15] Musso, E., Nicolodi, L.: A variational problem for surfaces in Laguerre geometry, Trans. Am. Math. Soc. 348, 4321-43227, 1996. 
[16] Musso, E., Nicolodi, L.: Laguerre geometry of surfaces with plane lines of curvature, Abh. Math. Sem. Univ. Hamburg, 69, 123-138, 1999.

[17] Nitsche, Johannes C.C.: Lectures on Minimal Surfaces, Volume 1, Springer-Verlag, Berlin, 1975.

[18] Palmer B.: Remarks on variation problem in Laguerre geometry, Rendiconti di Mathematica, Serie VII, vol.19, Roma, 281-293, 2000.

[19] Pinkall, U.: Dupin hypersurfaces, Math. Ann. 270, 427- 440, 1985.

[20] Rafaela F. do Prado, Roitman, P.: Laguerre minimal surfaces via congruence of lines, Bull. London Math. Soc., 1-11, 2012.

[21] Son, Yu P., Wang, Chang P.: Laguerre Minimal Surfaces in $\mathbb{R}^{3}$, Acta Math. Sin. 24, 1861-1870, 2008.

[22] Spivak, M.: A comprehensive introduction to Differential Geometry, Publish of Perish, Inc., Berkeley, 1979.

[23] Wang, C. : Weierstrass representation of Laguerre minimal surfaces in $\mathbb{R}^{3}$, Result. Math. 52, 399408, 2008.

[24] Warner, F. W.: Foundations of differentiable manifolds and Lie groups, Scott, Foresman and Company, Glenview, Illinois, 1971. 